\title{
Risk Assessment of Shell Eggs Internally Contaminated with Salmonella Enteritidis
}

\author{
Leanne M. DeWinter ${ }^{1, *}$, William H. Ross ${ }^{2}$, Hélène Couture ${ }^{1}$ and Jeff F. Farber ${ }^{1}$ \\ 1Bureau of Microbial Hazards, Food Directorate, Health Products and Food Branch, Health Canada \\ 2Bureau of Food Surveillance and Science Integration, Food Directorate, Health Products and Food Branch, Health Canada \\ *Corresponding author E-mail: Leanne.DeWinter@hc-sc.gc.ca
}

Received 9 May 2011; final version received 11 August 2011

Abstract A risk assessment was performed to determine the health risks associated with the consumption of Canadian grade A eggs internally contaminated with Salmonella Enteritidis. The distribution of the prevalence of contaminated eggs yielded an average of 1.7 per million from regulated laying flocks. The poorest storage and handling conditions for eggs represent $0.6 \%$ of exposures but result in $46 \%$ of illnesses; eggs handled under ideal storage and handling conditions account for $96 \%$ of exposures and represent $49 \%$ of illnesses. These findings suggest that risk management options targeting contaminated egg prevalence and the number of illnesses from a contaminated egg would be appropriate. Simulated risk management strategies included i) vaccination of flocks moving into houses previously occupied by positive flocks, ii) test and divert flock management strategy with environmental testing for $S$. Enteritidis, iii) eliminating the use of pooled shell eggs in foodservice and institutional settings, and iv) eliminating $S$. Enteritidis growth by improving egg storage and handling conditions. Strategies aimed at flock management yielded simulated reductions in contaminated egg prevalence between 2 and $29 \%$ of baseline, with smaller simulated gains from strategies aimed at reducing the number of illnesses per contaminated egg.

Keywords Salmonella Enteritidis eggs risk assessment

\section{Introduction}

Grade A table eggs offered for sale in Canada can come from several other sources: shell egg imports from the United States, hatchery surplus eggs from layer- and broiler-breeder flocks, and shell eggs from smaller flocks that are not regulated under the supply management system. Although these sources are an important consideration in the burden of illness of Salmonella enterica subsp. enterica serotype Enteritidis in table eggs, they are not included in this assessment, primarily due to a lack of information on flock prevalence and flock management practices.

There is only anecdotal information about many characteristics thought to influence the risk of $S$. Enteritidis illnesses from consuming shell eggs. No formal surveys have been done to establish the state of storage and handling conditions for eggs in Canada and most information used is an extrapolation from expert opinion, anecdotal information and from other countries' (United States) practices (e.g., sections 2.5 and 5.3). The baseline conditions selected followed the unpublished 2001 Health Canada risk assessment [1], except where updated information was available. Industry and consumer practices that relate to the risk management options that were examined are not well established. Therefore, the null case was selected as the baseline for these practices. 


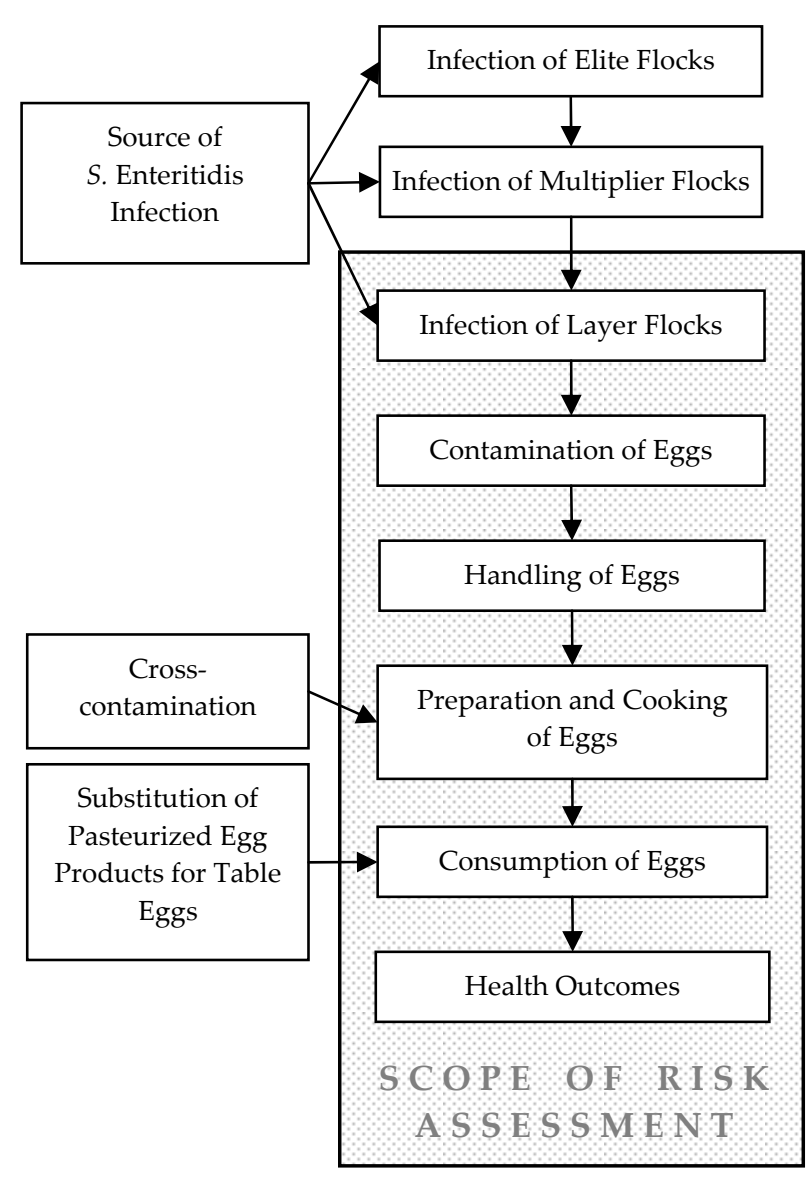

Figure 1. Scope of risk assessment.

Specific examination of the causes of flock infection was not done (Figure 1). Risk factors for horizontal infection -from environment to flock-were deemed well documented in the animal husbandry literature. Causes of vertical transmission from breeder flocks are managed by testing and control programs. Horizontal transmission from feed and water are managed by testing and control programs. Egg washing and application of sanitizers are already used to control external contamination on the egg shell and subsequent penetration to the egg contents. Cracked eggs and ungraded eggs are also excluded from consideration in this risk assessment.

This risk assessment describes the risk of illness from consumption of Canadian grade A table eggs internally contaminated with $S$. Enteritidis. It is based on Canadian flock management practices and Canadian egg storage and handling characteristics. Due to data availability regarding the prevalence of $S$. Enteritidis, the scope is limited to eggs produced by layer flocks regulated by the Egg Farmers of Canada (EFC), which represent approximately $97 \%$ of grade A table eggs (Figure 2). Risk outputs of interest in the risk characterization are the prevalence of internally contaminated S. Enteritidis eggs, the probability of illness from consuming a serving from a contaminated egg, the probability of illness from consuming a serving from an egg at random, and the number of illnesses. All bear direct relationship to human health outcomes.

The risk assessment and the resulting model developed allows for the simulation of the effects of risk management options. Effects on the baseline risk of illness are examined under simulations of three main risk management strategies: vaccination of laying flocks; egg diversion to pasteurization when flock environments test positive for S. Enteritidis; and modified table egg storage, handling, and preparation practices. Risk management options are evaluated by comparing the resulting risk outputs to the risk outputs from the baseline conditions.

The structure for the risk assessment follows Health Canada's Decision Making Framework [2] and the guidelines for a microbial risk assessment, as set out by the Codex Alimentarius Commission [3].

Supplementary data and information that has not been shown is available through the corresponding author.

\section{Hazard Identification}

Hazard identifications have been published in several risk assessments [1], [4], [14], which capture the characteristics of the pathogen and the epidemiological evidence for transmission of $S$. Enteritidis through eggs to humans.

\subsection{Salmonella}

Salmonella is an important foodborne pathogen worldwide, with approximately $95 \%$ of all salmonellosis cases resulting from ingestion of salmonellae in contaminated food [15]. This is followed by an incubation period, usually eight to 72 hours, during which the organism proliferates in the gut.

\subsection{S. Enteritidis and eggs}

For $S$. Enteritidis infections, eggs have been established as a major vehicle for human cases. Most serotypes of Salmonella contaminate shell eggs on the exterior shell surface, gaining entry to the interior contents by cracks in the shell or by other circumstances that can lead to eggshell penetration (e.g., time and temperature abuse, improper egg washing). Scientifically designed and tested egg washing protocols [16] used at grading stations serve to reduce the microbial load associated with egg shell surfaces, thereby reducing risk to consumers. $S$. Enteritidis is deemed to be an important food safety issue in shell eggs because of its ability to be present in internal egg contents due to trans-ovarian deposition of the organisms in egg contents as a result of the infected reproductive tissues of laying hens. Laying hens are most 
often infected with $S$. Enteritidis without showing any signs of illness or disease. The hazard is exacerbated when contaminated shell eggs are not held under conditions of refrigeration, allowing the organisms to access the nutrient rich yolk and grow rapidly. If an egg was internally contaminated with $S$. Enteritidis, most egg dishes are sufficiently cooked to inactivate $S$. Enteritidis; however, it would be present in raw egg dishes and some may survive in lightly cooked egg dishes.

\subsubsection{Epidemiological evidence}

Outbreaks of S. Enteritidis infections with associations with eggs or with egg-containing foods are well described in the literature. S. Enteritidis infections have been associated with eggs and egg products, but also with consumption of poultry and poultry products, with consumption of almonds and sprouted seeds, and by transmission from animals (pets) and humans. Infections acquired both domestically and from travel outside the country have been documented.

Literature reports of $S$. Enteritidis outbreaks associated with eggs may have decreased due to the fact that eggs are an established vehicle of $S$. Enteritidis. However, eggs will remain an important vehicle of infection due to transovarian transmission into intact eggs. This is evidenced by the recent outbreak of $S$. Enteritidis in shell eggs centred in Iowa, United States, which had an estimated 1939 associated illnesses [17]. In the United States, from 1985 through 2002, $73 \%$ to $80 \%$ of $S$. Enteritidis outbreaks have been identified as egg-related [18], [19]. Furthermore, a recent study by Voetsch et al. compared persons with $S$. Enteritidis to other Salmonella serotypes and identified consumption of undercooked eggs prepared outside the home as the strongest risk factor for acquiring sporadic illness [20].

In Canada, Salmonella has been the second most common enteric bacterial pathogen since at least 1997, judging by Salmonella isolates from both outbreaks and sporadic cases referred to the National Microbiology Laboratory from laboratory-confirmed cases [21] and cases reported through the National Notifiable Disease (NND) program [22]. National salmonellosis rates according to the NND registry were 18.0 and 16.4 per 100,000 for 2005 and 2006, respectively [23]. S. Enteritidis consistently ranks among the top three serovars associated with human illness in Canada since at least 1995, and the top five since at least 1983 [24], [26].

Compared to other serovars, the frequency of $S$. Enteritidis isolates has continued to increase year to year since 2003 and $S$. Enteritidis is now the most prevalent serotype: $28 \%$ of all salmonellosis cases in 2005 and $23 \%$ in 2006 [27], [28]. Laboratory-based data are useful for detailed strain characterization, whereas population-based rates from the NND registry are more accurate for overall salmonellosis figures because they are epidemiologically based and collected on a mandatory basis from public health units.

Applying Thomas et al. [29], [30] to data from Canadian population, laboratory and physician surveys [31], [32], the NND registry reports [23], salmonellosis surveillance [27], [28] and the microbiological and epidemiological literature ([29], [30]; data not shown) accounts for enteric disease underreporting when we synthesize how the number of $S$. Enteritidis associated illnesses in Canada varies from year to year. The estimated number of illnesses

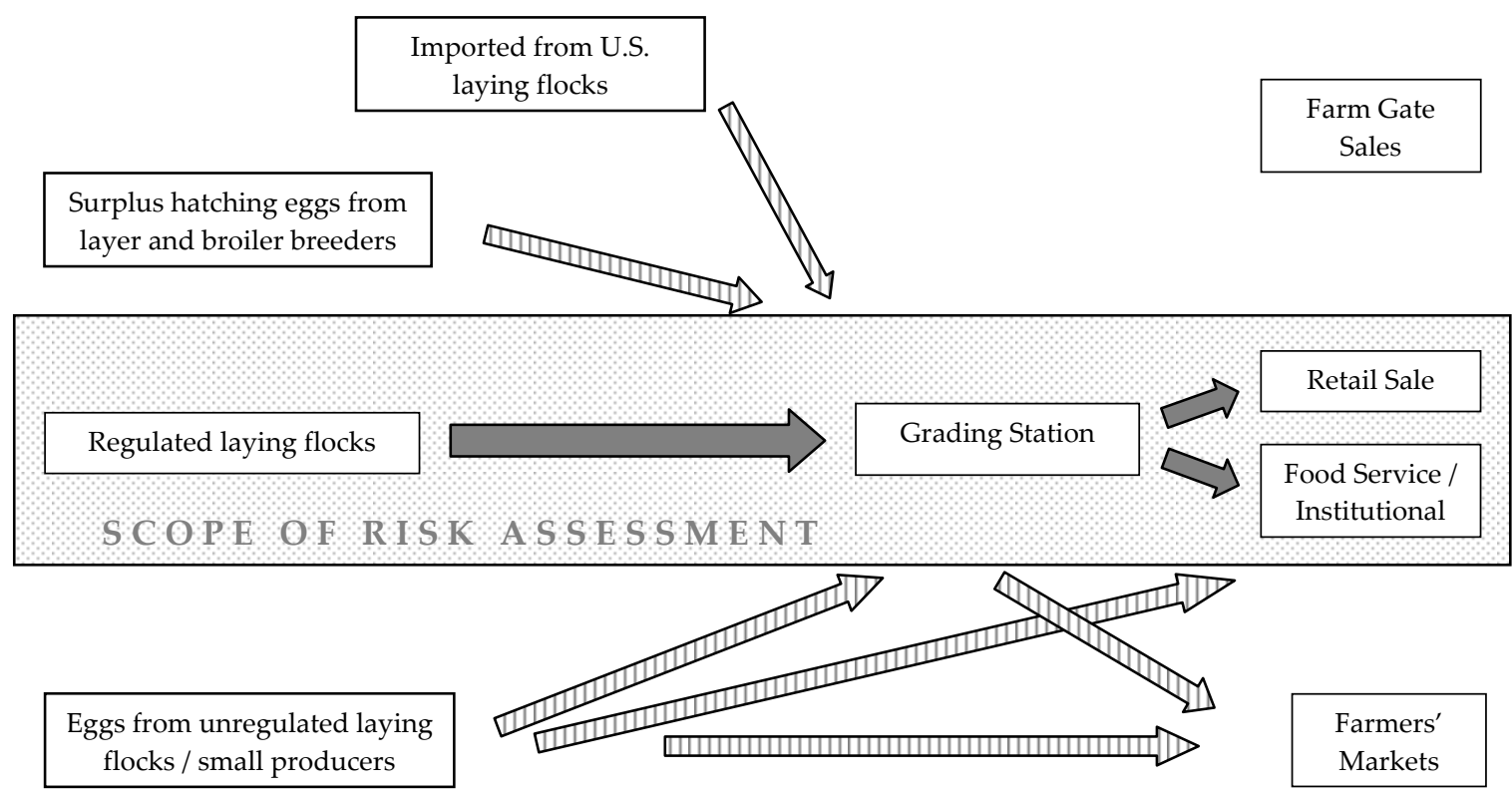

Figure 2. Scope of risk assessment for sources of grade A table eggs offered for sale in Canada. 
due to $S$. Enteritidis per year is a mean of 16,600 , with $5 \%$, $50 \%$, and $95 \%$ points of $15,300,16,500$, and 18,000 , respectively. We associate substantial uncertainty with each of those summary statistics. Some unknown portion of $S$. Enteritidis illnesses would be attributable to eggs from regulated flocks; other unknown portions would be attributable to eggs from other sources -other than grade A eggs from regulated flocks; eggs from broiler breeders, layer breeders, not regulated flocks and imported table egg; processed egg products -and still other unknown portions are attributable to the other pathways not included in this assessment.

\subsection{Relevant practices in the Canadian egg industry}

The Canadian egg industry has a number of practices that serve to decrease the prevalence of $S$. Enteritidis among the layer flocks and, in turn, the prevalence of eggs internally contaminated with $S$. Enteritidis.

The egg-type hatchery supply flocks have rarely been identified as a source of $S$. Enteritidis over the past ten years due to an industry-led program that comprises testing and control measures [36]. Furthermore, the CFIA performs routine surveillance at hatcheries by examining fluff samples every 6 weeks for salmonellae contamination and provides notification if samples are positive for $S$. Enteritidis so that appropriate action can be taken by the hatchery operator. There is an understanding that chicks known to be infected with $S$. Enteritidis will not be knowingly supplied to egg producers.

Most of the table egg sector in Canada is regulated by EFC and the egg marketing boards in the ten provinces and the Northwest Territories. Together they administer the production, pricing, marketing and promotion of eggs in Canada. Regulated layer flocks produce approximately $97 \%$ of eggs on the table egg market. There are about 1045 regulated egg farms and 19 million laying hens in Canada. Most of the regulated flocks are housed in cages, designed so the egg rolls out of the cage, away from the hen and the manure collecting equipment. Among the regulated flocks, there are also organic, free range, and free run flocks.

In 1990, EFC launched its "Safe from Salmonella" program, which was the first formal program in Canada to introduce biosecurity measures to primary commercial food production [37]. This program has evolved over time, with the incorporation of HACCP principles and renaming to Start Clean - Stay Clean ${ }^{\mathrm{TM}}$ in 1998, and completion of technical reviews by the CFIA in 2004 and 2007. The program includes on-farm inspections conducted by provincial and federal officers that provide each egg producer with a rating and suggestions for improvement. In general, features of the program are intended to minimize the possibility of introducing Salmonella to a laying flock, and include an all-in, all-out flock management style, cleaning and disinfection between flocks, environmental testing with diversion of eggs from positive flocks from the table egg market, drinking water quality monitoring, pest management programs, and provision of feed and litter from suppliers following Good Manufacturing Practices.

The program is industry-led and although participation in the program is voluntary for egg producers, participation is encouraged by offering compensation commensurate with the rating achieved in Start Clean Stay Clean ${ }^{\mathrm{TM}}$ for producers in the event that a layer flock tests positive for $S$. Enteritidis. Among the regulated layer flocks that operate under the Canadian supply management system, EFC achieved over 90\% participation in Start Clean - Stay Clean ${ }^{\mathrm{TM}}$ in 2005/2006, and this was expected to reach 98 to $100 \%$ in 2009. As of 2005/2006, approximately $92 \%$ of regulated layer flocks were being tested for $S$. Enteritidis, although testing has now achieved $100 \%$ participation. Currently, Start Clean - Stay Clean ${ }^{\mathrm{TM}}$ is implementing an insurance program that will provide for compensation when egg producers are faced with $S$. Enteritidis-infected flocks.

In 2005/2006, $92.4 \%$ of the layer flocks were tested for $S$. Enteritidis; however, adherence to environmental testing is voluntary and egg producers can withdraw participation at any time in some provinces. The sampling plans and testing protocols are provincially mandated and therefore variation exists in how the testing is practised. For example, the minimum environmental testing specified in any province is one test, which occurs 8 to 10 weeks before the end of the laying cycle. The various practices are summarized in Table 1 by describing the proportion of egg production covered by different practices [38]. The sensitivity of the different environmental testing schemes for detecting $S$. Enteritidis positive environments varies among the provinces and is unknown.

In Canada, flocks are not routinely vaccinated but in some jurisdictions may be vaccinated if the previously housed flock was found positive for $S$. Enteritidis. In 2006, for example, approximately $4 \%$ of the hens in regulated flocks were in vaccinated flocks [39], but further details such as the percentage of vaccinated flocks or the vaccination protocol were not available. Vaccination may be practised routinely, in the absence of information suggesting that a flock is at a higher risk of infection due to a particular source, or in a targeted fashion, with the knowledge of an infection in the previously housed flock. Targeted vaccination protects a flock deemed to be at higher risk of infection. These 
tend to be flocks being placed into an environment which tested positive during occupancy by the previous layer flock in case cleaning and disinfection did not completely eradicate $S$. Enteritidis, or in case $S$. Enteritidis could be reintroduced from the same source.

An important consideration for routine vaccination is that the number of organisms that infected birds shed in their faeces is decreased, meaning that infected flocks may become more difficult to detect using bacteriological testing using the same environmental testing protocol [40]. That the desired endpoint of decreased contaminated egg prevalence would likely be met is demonstrated by the results of vaccination programs in the United Kingdom, where implementation of the control measure of vaccinating layer flocks is thought to have led to a dramatic decline in the number of cases of $S$. Enteritidis [41].

Another consideration is that vaccination precludes serological testing of birds, since the antibodies produced in response to vaccination cannot be distinguished from those produced in response to a natural infection. However, this disadvantage is not critical because serological testing is usually performed at the level of breeding stock, but rarely, if ever, at the level of commercial layer flocks in Canada. It has also been reported that neither inactivated nor live attenuated Salmonella vaccines have consistently prevented infection in laying hens, especially against high dose challenges [42].

\begin{tabular}{|c|c|}
\hline \multicolumn{1}{|c|}{ Flock testing practice } & $\begin{array}{c}\text { Coverage of egg } \\
\text { production (\%) }\end{array}$ \\
\hline $\begin{array}{l}\text { At least one environmental test of pullet } \\
\text { flock }\end{array}$ & 57.5 \\
\hline Frequency of testing during laying cycle & \\
\hline Once & 82.5 \\
\hline Four times & 17.5 \\
\hline Number of sites sampled & \\
\hline Not specified & 4.2 \\
\hline $40-59$ & 56.6 \\
\hline $60-64$ & 39.2 \\
\hline Type of samples & 46.5 \\
\hline Swabs & 42.7 \\
\hline Dust, fluff, etc. & 10.9 \\
\hline Combinations of the two & \\
\hline Pooling of samples & 39.9 \\
\hline $2-4$ & 57.5 \\
\hline 5 & 2.6 \\
\hline 16 & \\
\hline
\end{tabular}

Table 1. National laying flock testing practices in 2005 and 2006 represented by summing provincial egg production for each practice.
A small proportion of table eggs in Canada originate from layer- and broiler-breeder surplus hatching eggs, smaller producers that are not regulated under the supply management system, and from United States shell egg imports. Although these sources can also contribute to the burden of illness due to $S$. Enteritidis in table eggs, they are out of the scope of this risk assessment due to data limitations.

Eggs may be graded only at federally registered grading stations, which must meet requirements, such as temperature and humidity controls for storage of eggs, specified under the Egg Regulations [16]. Grading stations are monitored regularly by the CFIA, and twice per year undergo environmental sampling for salmonellae. Positives are serotyped and corrective actions are required. The parameters for egg washing at the federally registered grading stations are specified under the Egg Regulations to prevent the contamination of egg contents with shell contaminants, including dictating the use of sanitizers, temperature for spray washes, and the temperature and $\mathrm{pH}$ for recirculating systems.

\subsection{Mechanism of S. Enteritidis internal contamination of eggs}

Deposition of $S$. Enteritidis directly into the egg contents is thought to occur primarily in the albumen, typically at a site near the vitelline membrane [43], [44], [45]. The albumen is separated from the yolk by the vitelline membrane, but the vitelline membrane is semi-permeable and might permit limited access to nutrients in the yolk that are capable of diffusing through the membrane. It is generally thought that $S$. Enteritidis grows slowly and only to a limited extent in the albumen [46], [47]. Deposition directly into the yolk contents is thought to occur only very rarely or not at all [45].

Eggs can be externally contaminated with salmonellae at the point of lay from contact with feces, or after lay from contact with a variety of sources including dust, litter, nesting materials, egg conveyances, and through contact with surfaces, equipment, or personnel during the grading and packaging environments. There is no evidence to suggest that $S$. Enteritidis can move through eggshells and the internal membranes more readily than other salmonellae, and S. Enteritidis is not considered to compete effectively with other organisms on shells.

\subsection{Prevalence of S. Enteritidis}

\subsubsection{Prevalence of S. Enteritidis infected layer flocks}

$S$. Enteritidis has been isolated from the environments of Canadian layer flocks pursuant to outbreak investigations, validation of an epidemiological link 


\begin{tabular}{|l|c|c|c|c|c|}
\hline \multicolumn{1}{|c|}{ Source } & Year of testing & Flocks tested & $\begin{array}{c}\text { Hens in } \\
\text { tested flocks }\end{array}$ & $\begin{array}{c}\text { Flocks } \\
\text { positive }\end{array}$ & $\begin{array}{c}\text { Hens in } \\
\text { positive flocks }\end{array}$ \\
\hline Poppe et al. (1991a) [48] & 1989 & 295 & & 8 & \\
\hline CEMA (2007a) [39] & 2005 excl. BC & 1550 & 15533731 & 12 & 154664 \\
\hline CEMA (2007b) [54] & 2006 & 1437 & 20250807 & 4 & 137940 \\
\hline
\end{tabular}

Table 2. S. Enteritidis layer flock envrinomental testing results in Canada.

between illness and an implicated food, and through routine monitoring and testing programmes.

Data demonstrating $S$. Enteritidis prevalence among all layer flocks producing table eggs for the Canadian market are unavailable, particularly for those sources with small proportions of the market share, such as small unregulated layer flocks; data demonstrating prevalence among layer flocks producing table eggs for export to Canada are unavailable.

Published data regarding the results of $S$. Enteritidis testing for layer flocks in Canada are limited to regulated flocks or commercial flocks and include microbiological literature [48], [49], annual reports from Provincial and national Egg Marketing Boards and Egg Producers, Breeders Associations, and government reports [39], [50], [54], some of which is captured in Table 2. Additional information about $S$. Enteritidis in Canadian poultry, broiler, broiler breeder and layer breeder flocks [55]-[57] and information about $S$. Enteritidis flock prevalence from other countries is deemed to be not relevant to describing flock prevalence in Canada.

Current practice among regulated layer flocks specifies that the determination that a layer flock is infected with $S$. Enteritidis is based on the results of environmental testing, which is consistent with published findings [14], [58], [60]. Studies have demonstrated that flocks inhabiting a negative environment rarely contain infected birds, meaning that the predictive value of a negative result is good [49], [61], when environmental test sensitivity is sufficiently high. The presence of $S$. Enteritidis in the environment of a layer flock indicates intestinal colonization, a precursor of reproductive tract infection, and is therefore an indicator of flock infection [59]. On the other hand, further testing of flocks in a positive environment does not always demonstrate infected birds [49], possibly because reproductive tract infection can persist after intestinal colonization ceases [62], [63].

Published data in the microbiological literature regarding prevalence of $S$. Enteritidis in Canadian domestic poultry flocks [48] are outdated in light of flock management practices introduced since then.
There are limited data from provincial egg marketing boards:

- Quebec has regulations pertaining to testing requirements for reducing the incidence of S. Enteritidis in table eggs (LRQ cM-35.1 a.92); the 2005/2006 Rapport Annuel de la Fédération des Producteurs d'Oeufs de Consommation du Québec indicated that the rate of environmental $S$. Enteritidis contamination of layer houses was $1.64 \%, 2.26 \%$, and $0 \%$ in each of 2003, 2004, and 2005 respectively, compared to overall Salmonella spp. contamination rates of $72.13 \%, 71.94 \%$, and $75.32 \%$ during the same time periods. Environmental contamination in pulletrearing houses was $2.61 \%$ in 2003 and $1.14 \%$ in 2005, compared to general salmonellae contamination rates of $52.17 \%, 49.59 \%$, and $74.43 \%$ in 2003,2004 , and 2005 respectively.

- The 2004 and 2005 Annual Reports of the Ontario Egg Producers indicate that compensation for "Salmonella infected flocks", which covers vaccination costs and compensation for destroyed birds, was 0 in 2003, $\$ 20,637$ in 2004, and \$110,290 in 2005.

- Annual reports from 2003 and 2004 indicate the onset of isolation of $S$. Enteritidis in the environments of Saskatchewan layer flocks [50], [64]. Rates of general salmonellae contamination in the layer house environment ranged from a low of $13.6 \%$ in 2001 to a high of $36.1 \%$ in 1997 during 1997-2004. Saskatchewan has also passed a Risk Management Order through the Saskatchewan Gazette (2006) outlining testing requirements for table egg layer flocks and measures to be followed when $S$. Enteritidis is identified in a production facility.

There is limited information about $S$. Enteritidis testing in broiler breeder and layer breeder flocks [57]; (data not shown) and no information about $S$. Enteritidis testing among not regulated flocks.

Potential for seasonal variation, regional variation, and variation with flock management practices are demonstrated in information from other evaluations [6], [8], but have not been measured in available data for Canada. 


\subsubsection{Prevalence of eggs internally contaminated with S. Enteritidis}

Several studies have characterized the proportion of contaminated eggs from naturally infected flocks [8], [49], [61], [65]-[69]. Humphrey et al. [65] monitored infected hens' production over a period of approximately four months, noting that their production of internally contaminated eggs is intermittent and at low prevalence. The dynamics of contaminated egg prevalence in an infected flock are only very generally characterized [13], and thus are inadequately described. The egg within flock prevalence used here follows that reported in Poppe et al. [49] and USDA [8].

Flock vaccination is thought to reduce intestinal colonization, a precursor to infection of the reproductive tissues, and therefore results in a lower rate of production of contaminated eggs. Killed vaccines, or bacterins, are thought to stimulate humoral immunity whereas live vaccines activate cell-mediated immunity; the latter is desirable for use in layer flocks since salmonellae are intracellular parasites. On the other hand, there are some studies showing that bacterins elicit an antibody response to flagella, which may prevent colonization and infection of the bird [70]. S. Enteritidis egg prevalence from $S$. Enteritidis positive vaccinated flocks was found to be one-quarter of that from $S$. Enteritidis positive nonvaccinated flocks $(0.24 \%$ versus $1.0 \%$, respectively), when vaccinated flocks were injected intramuscularly at four and eighteen weeks of age with a commercial adjuvanated killed vaccine based on a virulent $S$. Enteritidis PT4 strain [69].

\subsection{Impact of storage and handling conditions on S. Enteritidis levels}

\subsubsection{Initial S. Enteritidis levels}

Studies on naturally [71] and artificially [72] infected hens indicate that fresh contaminated eggs contain few S. Enteritidis. Contaminated eggs recently laid by naturally infected hens had no more than 20 organisms with a mean of 7 organisms, whereas contaminated eggs recently laid by artificially infected hens had a mean number of 200 organisms. In addition to the route of infection, differences in the invasiveness of the strains might also have an effect on the size of the initial populations at lay.

It is hypothesized that there is approximately $1 \log$ of growth of $S$. Enteritidis before the $\mathrm{pH}$ of the albumen rises and local nutrients are exhausted [73], [74].

\subsubsection{Yolk membrane breakdown and S. Enteritidis growth}

$S$. Enteritidis grows slowly and to a limited extent in the albumen [46], [47], but once reaching the yolk, very rapid growth can occur under permissive temperatures.

S. Enteritidis is thought to gain access to the yolk as the viscosity of the albumen decreases and the permeability of the vitelline membrane increases; these events occur naturally over time [73]. Higher temperatures and longer storage times generally favour loss of membrane integrity and $S$. Enteritidis crossing the vitelline membrane before the egg is consumed [75]. Paoli's [1] or Whiting and coworkers' [5] analysis of experimental data from Humphrey (experimental data, unpublished) describes the rate of yolk membrane breakdown.

If at some point during the handling of a contaminated egg, the cumulative percentage loss in membrane integrity reaches $100 \%, S$. Enteritidis is assumed to have sufficient nutrients to grow whenever storage temperature is above minimum growth temperature. If the egg temperature is maintained at a sufficiently low level for the entire period of time following loss of membrane integrity, up to the point of consumption, $S$. Enteritidis is not expected to grow.

The minimum growth temperature is thought to be between $6^{\circ} \mathrm{C}$ and $8^{\circ} \mathrm{C}$, but is not known with precision and is likely to vary among eggs and among $S$. Enteritidis strains. Membrane breakdown followed by storage above the minimum growth temperature can result in growth over time to a maximum population of $10^{10}$ organisms per egg [6], [8], [76], [77], [78].

After eggs are broken, access to yolk nutrients permits $S$. Enteritidis growth, subject to minimum temperature conditions. Growth can also occur among the $S$. Enteritidis that survive cooking when prepared egg dishes are held after cooking before consumption.

\subsubsection{Storage and handling time and temperature}

Based on the Canadian egg industry and the previous unpublished risk assessment [1], the production and handling of table eggs was separated into thirteen sequential stages, from point-of-lay through to storage after preparation of eggs for consumption; distributions characterizing time and temperature were developed for each stage (data not shown). We substitute pre-grading storage temperature that reflects the regulatory change requiring the storage of eggs at $10^{\circ} \mathrm{C}$ (Egg Regulations, C.R.C., c. 284, 2009). Times and temperatures in this synopsis reflect the variability in storage and handling time and temperature for individual eggs. 
- Barn: approximately $75 \%$ of eggs come from operations that collect eggs twice daily -eggs remain in the barn between $1 \mathrm{~h}$ and $12 \mathrm{~h}$ - and the rest of eggs come from operations that collect eggs once daily -they remain in the barn between $1 \mathrm{~h}$ and $24 \mathrm{~h}$; barn temperatures are between $18^{\circ} \mathrm{C}$ and $40^{\circ} \mathrm{C}$.

- On-farm: at any time, between $90 \%$ and $95 \%$ of eggs come from producers who follow recommendations for cold storage, at temperatures between $10^{\circ} \mathrm{C}$ and $14^{\circ} \mathrm{C}$; the other $5 \%-10 \%$ of eggs are stored at room temperature, $15^{\circ} \mathrm{C}$ to $25^{\circ} \mathrm{C}$, before transport to grading; $60 \%-80 \%$ of eggs are picked up from farms at a rate of 2-3 times per week, after 0-4 days storage, while the other $20 \%-40 \%$ of eggs are picked up from farms at a rate of 1-2 times per week, after 0-7 days storage.

- Transport to grading: transport to pre-grading takes $1 / 2 \mathrm{~h}-8 \mathrm{~h}$, with no change in individual egg temperatures.

- Pre-grading storage: eggs remain in pre-grading storage from $1 \mathrm{~h}-24 \mathrm{~h}$, at temperatures between $4^{\circ} \mathrm{C}$ and $10^{\circ} \mathrm{C}$ (by regulation), or, for $1 \%$ of eggs, at temperatures between $10^{\circ} \mathrm{C}$ and $15^{\circ} \mathrm{C}$ (previous regulations).

- Grading and re-packing: eggs transverse grading in $0.1 \mathrm{~h}-1 / 2 \mathrm{~h}$, at temperatures of $15^{\circ} \mathrm{C}-25^{\circ} \mathrm{C}$.

- Post-grading storage: eggs remain in post-grading storage from $1 / 2$ days-7 days, at the pre-grading storage temperature.

- Transport to wholesale and retail: $60 \%-80 \%$ of eggs travel directly to retail stores; the other $20 \%-40 \%$ of eggs go to wholesale storage first; transportation time is $1 \mathrm{~h}-6 \mathrm{~h}$.

- Wholesale storage: when it occurs, wholesale storage is at temperatures of $2^{\circ} \mathrm{C}-10^{\circ} \mathrm{C}$ for $1 / 2$ days- 21 days.

- Retail storage deviations: $0.2 \%-1 \frac{1}{2} \%$ of eggs are held for $1 \mathrm{~h}-12 \mathrm{~h}$ at temperatures between $15^{\circ} \mathrm{C}$ and $30^{\circ} \mathrm{C}$, while waiting for refrigerated storage at retail.

- Retail storage: at retail, egg storage temperatures are between $4^{\circ} \mathrm{C}$ and $12^{\circ} \mathrm{C}$, with sales, generally by the dozen, between $1 / 2$ days and 30 days, before 7 days on average.

- Consumer storage: while $1 / 2 \%-2 \%$ of eggs are stored at room temperature, most eggs are stored at refrigerator temperatures, $4^{\circ} \mathrm{C}$ to $12^{\circ} \mathrm{C}$; carton storage time is between 1 day and 60 days, with mean time approximately 14 days; individual egg storage varies between 0 days and the carton storage time (the last egg(s) used).

- Post-break, pre-cook storage: at home settings, 1\%$10 \%$ of eggs are broken, pooled and held for $1 \mathrm{~h}-6 \mathrm{~h}$ at room temperature, $15^{\circ} \mathrm{C}-25^{\circ} \mathrm{C}$ for $5 \%-20 \%$ of eggs, or at refrigerator temperature, $4^{\circ} \mathrm{C}-12^{\circ} \mathrm{C}$, for the rest; at FSI settings, $1 \%-30 \%$ of eggs are broken, pooled and held for $1 \mathrm{~h}-24 \mathrm{~h}$, at room temperature for $25 \%$ -
$75 \%$ of the eggs or at refrigerator temperatures, $4^{\circ} \mathrm{C}$ $12^{\circ} \mathrm{C}$, for the rest.

- Post-cook storage: at home settings, $1 \%-10 \%$ of prepared egg dishes are stored after preparation for $1 \mathrm{~h}-24 \mathrm{~h}$, at room temperature for $1 \%-10 \%$ of those dishes or at refrigerator temperature for the rest, before consumption; at FSI settings, $1 \%-30 \%$ of egg recipes are stored for $0 \mathrm{~h}-24 \mathrm{~h}$, at room temperature for $5 \%-20 \%$ of the eggs and at refrigerator temperature for the rest, before consumption.

\subsubsection{Cooking effects}

Despite the heat susceptibility of $S$. Enteritidis, consumers are at increased risk of exposure due to preferences for dishes containing raw or lightly cooked eggs. These preparation styles include those intended to maintain a liquid yolk, such as in 'sunny-side-up', 'soft-boiled' or 'poached' eggs. Studies indicate smaller $\log _{10}$ reductions in $S$. Enteritidis when eggs are prepared those ways and larger reductions for eggs fried 'over-easy', scrambled or hard-boiled. Cooking for eggs used in recipes can consist of a continuum of cooking, from several hours of baking (well-cooked), to sauces (lightly-cooked) to inclusion of raw egg without further cooking (dressings) [1], [4], [8], [10], [79], [85].

\subsubsection{Egg preparation practices and egg pooling}

Pooled eggs, or multiple broken shell eggs combined, can be stored for some time before use in a recipe or meal. This is a practice, considered to be more common in FSI cooking, where a relatively large number of eggs are broken and stored in a large pool. Current use of processed egg products rather than pooled shell eggs at home or FSI settings is unknown.

\subsection{Consumption of table eggs}

The total production of eggs in Canada was approximately 6.3 billion in 2005, of which approximately $75 \%$ were sold as table eggs [86]. The remaining eggs were used for processed egg products for domestic or export markets.

\section{Hazard Characterization}

This section draws on the microbiological and epidemiological literature about the nature of the health outcome of illness due to $S$. Enteritidis and the factors that determine the relationship between the amount of the pathogen ingested and the host response in normal and susceptible populations. 


\subsection{Health outcome}

Symptoms of salmonellosis range from mild to severe diarrhoea, abdominal pain and vomiting, and fever accompanied by headache and chills. In cases of severe diarrhoea, dehydration may lead to hypotension, cramps, oliguria and uraemia. The vast majority of cases comprise acute illness that lasts a few days and recovery within a week. Small proportions of cases develop bacteraemia or meningitis, or may develop localized infections causing abscesses, arthritis, cholecystitis, endocarditis, pericarditis, or pneumonia, and death is uncommon. Invasive infections and death are more common in the very young, very old or immunocompromised [87]-[91]. Long-term sequelae resulting from salmonellosis can include malabsorption of essential nutrients (which can result in compromise of the immune system and may lead to infection), allergy, reactive arthritis, autoimmune disorders, and neoplasia. Although reactive arthritis is usually of short duration, some patients may develop chronic disease and patients with certain underlying conditions (e.g., haemoglobin abnormalities) may be prone to complications.

Several analyses of non-typhoid salmonellosis have indicated that $S$. Enteritidis may be slightly less likely than other serovars to result in invasive infections as a result of gastroenteric infections except possibly in susceptible groups, and no more likely than other serovars to result in death [79], [87], [89], [92].

Faecal excretion of salmonellae normally continues for a few weeks after recovery. In children and some individuals with asymptomatic infection, excretion may continue for months or, rarely, years. In less than $1 \%$ of cases, individuals become chronic carriers and continue to excrete for more than a year. Treatment of acute Salmonella gastroenteritis with antimicrobial agents has been discouraged since the illness is usually relatively brief in duration and self-limiting. Furthermore, antimicrobial agents are thought to have little or no effect on the course of infection and may even prolong excretion or carriage of the organism. Antimicrobial therapy is indicated for patients with systemic infection or very severe illness.

\subsection{Susceptible and normal populations}

Health Canada's approach to risk assessment identifies differences in the probability of infection from consuming the same number of $S$. Enteritidis among susceptible populations and normal populations of individuals. For $S$. Enteritidis, a single susceptible population comprises all those individuals at higher risk of salmonellosis: newborns, young children, pregnant women, elderly individuals and immunocompromised individuals. A single normal population includes the remainder of the individuals in the general population. Anecdotal information and consideration of age, prevalence of immunocompromising conditions and pathogen virulence suggest that the size of the susceptible population varies between $15 \%$ and $25 \%$ of the whole population.

\subsection{Dose-response assessment}

The dose-response assessment provides a mathematical link between the number of $S$. Enteritidis organisms consumed and the fraction of a consuming population that would become ill, with the characteristic that the probability that an individual at random from the consuming population becomes ill decreases, without threshold, as the ingested dose decreases.

This dose-response assessment uses the Weibull doseresponse function,

$$
\pi(d ; \theta, b)=1-\exp \left(-\theta d^{-b}\right)
$$

where $\pi(d)$ is the probability of illness from ingesting a dose $d S$. Enteritidis, $\theta$ acts like a location parameter and $b$ acts like a shape parameter. With derivation [1], [93] (data not shown), the specific parameterization follows:

- a meta-analysis of feeding trial data suggests that a Normal(-1.22, .0252) describes how $\ln b$ varies among pathogens;

- a value for

$$
\theta=-\ln (1-P) X^{-b}
$$

follows from the attack rate, $6.6 \%$, in a large $S$. Enteritidis in ice cream outbreak [94], [95], the S. Enteritidis concentration (cfu per g) in contaminated ice cream servings (logNormal, mean .15 and standard deviation .1) and the amount (g) of ice cream consumed (Pert(60,130,260)); and,

- a value for

$$
\theta_{\text {susc }}=\theta_{\text {normal }}\left[\frac{\ln \left[1-\operatorname{Beta}\left(a_{s}, b_{s}\right)\right]}{\ln \left[1-\operatorname{Beta}\left(a_{n}, b_{n}\right)\right]}\right]
$$

where $a_{s}=231, b_{s}=987, a_{n}=749$ and $b_{n}=5966$, given the different attack rates among 1208 exposed children and 6715 adults in a waterborne S. Typhimurium outbreak in Riverside, California [96].

For an individual at random from a susceptible population, the probability of illness is higher than for an individual at random from a normal population:

- approximately 1.7 times higher at doses in the range $1 \mathrm{~S}$. Enteritidis to $10^{3} \mathrm{~S}$. Enteritidis; and, 
- approximately the same at doses $>10^{7.5} \mathrm{~S}$. Enteritidis, where probability of illness approaches 1 for normal and susceptible individuals.

There remains considerable discussion about the shape and parameterization of a dose-response function for S. Enteritidis. S. Enteritidis in eggs risk assessments have used various forms for the dose response function, including the Beta-Poisson dose response function [5]-[8], [11], with various parameterizations, some of which also distinguish between susceptible and normal populations [5], [6] and others that do not [7], [8], [11]. Other risk assessments have used models that distinguish illness severity but not dose-response differences between susceptible and normal populations [7]. Health outcome endpoints vary slightly, but not substantially, among the risk assessments. Figure 3 illustrates the different doseresponse models' representation of how the probability of illness changes between populations and with S. Enteritidis dose ingested.

At low S. Enteritidis doses, the Weibull model used in this risk assessment is more concervative than the BetaPoisson dose-response models that FAO/WHO (Mid) [7], USDA (m.l.e.) [8] and Thomas et al. [11] derived from the same data set of outbreaks. For the normal population, it attributes higher probability of illness at low doses (1-100 S. Enteritidis) and higher probability of illness at high doses $\left(\geq 10^{5.5} \mathrm{~S}\right.$. Enteritidis). For the susceptible population, it attributes higher probability of illness at all S. Enteritidis doses. It attributes lower probability of illness, at all $S$. Enteritidis doses, than the Whiting et al. model [5].

The dose-response model used in this risk assessment suggests virtually $100 \%$ attack rate at $S$. Enteritidis doses above approximately $10^{7} \quad$ S. Enteritidis (susceptible population) and $10^{8} \mathrm{~S}$. Enteritidis (normal population). The FAO/WHO (Mid), USDA (m.l.e.) and Thomas et al. dose-response models confer immunity to approximately $8 \%$ of the population even at $10^{10} \mathrm{~S}$. Enteritidis doses [7], [8], [11].

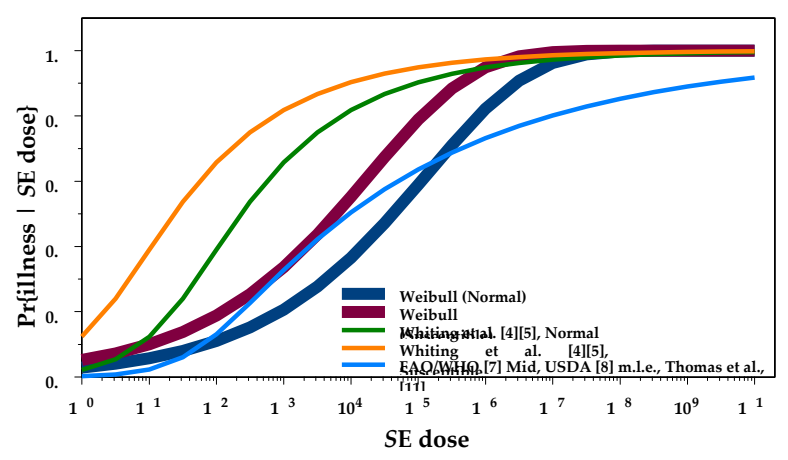

Figure 3. Probability of illness with $S$. Enteritidis dose ingested according to different dose-response models.

\subsection{Severity of illness}

Apportionment of illnesses among all individuals to minor illness, severe illness and death follows the USDA [8] apportionment of illness severity (at mean levels) to $96 \%$ minor, $4.4 \%$ severe (hospitalization and chronic sequelae) and $0.041 \%$ death.

\section{Exposure Assessment}

The exposure assessment describes the frequency of consumers' exposure and the number of $S$. Enteritidis consumed in egg servings prepared from eggs internally contaminated with $S$. Enteritidis. Outputs of interest from the exposure assessment are the prevalence of $S$. Enteritidis contaminated eggs and $S$. Enteritidis ingested in a contaminated egg serving, which are necessary to complete risk characterization (section 5) and to examine risk management options (section 6, 6.2).

Exposure is estimated using a simulation model originally developed for Health Canada [1] that applies structural formula to a set of model inputs (data not shown). The model inputs reflect data updates to flock prevalence, new information from the animal husbandry and microbiological literature, marketing and production information, some handling and storage times and temperatures, meal and recipe allocation among individuals, and cooking $\log _{10}$ reductions.

The section is organized as follows:

- section 4.1 establishes the baseline conditions for the exposure assessment;

- section 4.2 reports exposure outputs from those baseline conditions;

o section 4.2.1 reports results for baseline conditions; and,

o section 4.2.2 reports results for special cases and sensitivity.

\subsection{Baseline conditions}

\subsubsection{Prevalence of S. Enteritidis contaminated eggs consumed}

The prevalence of $S$. Enteritidis contaminated eggs among table eggs consumed is synthesized from information about flock prevalence, prevalence of $S$. Enteritidis positive eggs from a positive flock, the size of positive flocks, the number of eggs produced and the fraction that are sold for the table egg market.

\section{Prevalence of $S$. Enteritidis infected layer flocks}

Available data come from environmental testing of the regulated laying flocks during two years (Table 2). However, flock environment test sensitivity for detecting $S$. Enteritidis across that testing and the presence in the 
Canadian layer flock population of low and high risk factors for S. Enteritidis-infection (section 2.5.1) is not known and not otherwise accounted for.

The baseline specification for flock prevalence is consistent with those results (data not shown), and we use it to describe how flock prevalence varies among years. Information that describes the potential for seasonal variation, regional variation, variation with flock management practices, and variability in flock prevalence across different flock sizes is not available.

Despite a lack of experimental data on variability in flock prevalence across flock size, this special case was considered in section 4.2.2 using alternative specifications.

\section{Within-flock egg prevalence}

Baseline specification for how the prevalence of $S$. Enteritidis contaminated eggs (egg prevalence) laid in a positive flock environment varies (data not shown) is informed by the egg prevalence reported in Poppe et al. and USDA [8], [49].

Baseline conditions do not account for vaccination effects; vaccination effects are considered separately as a risk mitigation strategy (section 6.1.1). While policies mandate that eggs from $S$. Enteritidis positive flocks be diverted to pasteurization, current practices vary among jurisdictions and thus this factor is considered only under examination of risk management options (section 6.1.2).

Alternative specifications for within-flock prevalence are considered in section 4.2.2.

\section{Size of $S$. Enteritidis positive flocks}

The flock size distribution (data not shown) is used as a simple surrogate to describe how the number of eggs laid in a $S$. Enteritidis positive flock environment varies and how that affects how much overall egg prevalence among table eggs varies under the same flock and within flock prevalence.

\section{Egg production}

Baseline specification fixes production statistics at 2005 levels (AAFC, 2006; section 2.7).

\subsubsection{Initial level of S. Enteritidis contamination of eggs}

The initial deposition of $S$. Enteritidis in the albumen at a site near the vitelline membrane, varies with a mean of 7 $S$. Enteritidis per egg, followed by up to $1.5 \log 10$ growth, after which the population is considered stable.

Alternatives' descriptions of the $S$. Enteritidis deposition in contaminated eggs are considered in section 4.2.2.

\subsubsection{Egg storage, handling and preparation}

\section{Storage and handling times and temperatures}

Time- and temperature-related conditions change vitelline membrane permeability and permit growth when $100 \%$ yolk membrane breakdown occurs. Baseline specification describes how production, storage and handling conditions vary among eggs. Following the previous risk assessment [1], we simplified them by a set of sequential stages' times and temperatures (section 2.6.3; data not shown) and we set the same storage and handling conditions of eggs eaten in home settings and in FSI settings. We impute the temperature of the environment in which the egg is held to the egg contents and we ignore the heating or cooling that occurs during transition from an environment with one temperature to an environment with another temperature.

Alternative storage and handling characteristics are considered separately under examination of risk management options (section 6.2.1).

\section{Yolk membrane breakdown and $S$. Enteritidis growth} The rate of yolk membrane breakdown increases with temperature and varies among individual eggs (data not shown). Baseline conditions include the fixed, but uncertain, maximum time to $100 \%$ yolk membrane breakdown from Paoli [1].

After $100 \%$ yolk membrane breakdown occurs, $S$. Enteritidis populations grow if the storage temperature is above minimum growth temperature. Minimum growth temperature varies -by $S$. Enteritidis and egg-between $6^{\circ} \mathrm{C}$ and $8^{\circ} \mathrm{C}$. Growth rate increases with temperature and varies also with $S$. Enteritidis and egg. Maximum population density for $S$. Enteritidis in eggs is $10^{10} \mathrm{cfu}$ per egg.

\section{Cooking}

Baseline specification for how much cooking reduces $S$. Enteritidis populations in an egg meal or recipe follows several precedents' specifications [1], [4], [8], [10] (section 2.6.4), generally as a $\log _{10}$ reduction.

Inactivation varies among different types of egg meals, in which egg is the principal distinguishable component as in fried eggs, omelettes, soft-boiled, poached and hard-boiled eggs, and among different egg recipes, in which egg is generally not a distinguishable component as in dressings, sauces, drinks, and baked products. For egg recipes, we simplify the variation into among-recipe type categories and within egg recipe type categories components. These components capture how $\log _{10}$ reductions vary between raw, lightly cooked and wellcooked eggs and how $\log _{10}$ reductions vary among 
applications of recipes within a recipe type category (data not shown), respectively.

\section{Egg preparation practices}

Inferences from the 24-hour recall data in a series of Federal-Provincial Nutrition Surveys conducted from 1991 to 1999 [97] inform the baseline specification about how individuals' preferences in preparation and consumption of eggs vary for egg meals versus egg recipes, among egg meal types and among egg recipe types among individuals within population groups and between home and FSI settings (data not shown).

Individuals in normal and susceptible groups as described in section 3.2 are not explicitly identified in the Federal-Provincial Nutrition Surveys data [97]. We impute to susceptible populations of individuals, the consumption characteristics of populations of individuals with the same nominal age-sex characteristics that section 3.2 attributes to susceptible individuals; we impute to normal populations of individuals, the consumption characteristics of populations of individuals with the same nominal agesex characteristics that section 6.2.3 attributes to normal individuals.

Effects of changes to egg preparation practices are considered as risk management options in section 6.2.2.

\section{Egg pooling}

Egg pooling frequency and egg pool size variability are known only to the extent that egg pooling before final preparation or consumption into egg dishes (scrambled eggs, omelettes, hard-boiled eggs in egg salad, sauces and dressings, casseroles, and baked products) is a practice that happens more frequently in FSI settings than in home settings and that egg pools in FSI settings are larger than in home settings [1], [8].

Following Paoli [1] and USDA [8], we simplify the practices to represent the number of individuals that would consume a serving from an egg meal or recipe made with pooled eggs (data not shown).

Effects of changes to pooling and preparation practices are considered as risk management options in section 6.1.2.

\section{Growth during preparation}

Sometimes, eggs are broken and held at room or refrigerator temperature before cooking. Also, prepared egg dishes are sometimes held at room or refrigerator temperature before serving. In both cases, $S$. Enteritidis growth occurs (data not shown).

\subsection{Exposure assessment results}

Except where pertinent to discussions, intermediate outputs from the exposure assessment - display of model inputs and intermediate outputs - are not reported. Model inputs are specified by distributions of values rather than single values. They describe how the characteristic that the input describes varies among individuals, conditions, or circumstances, or they describe uncertainty about that variability. Model outputs reflect those distribution inputs. Reporting output variability is done by

- separating results by breakdowns of interest: for home settings and FSI settings, among egg meal and egg recipe types and among recognized $S$. Enteritidis growth scenarios; and,

- assessing variability of results within those breakdowns: summary statistics from the distribution of values that describes how results, typically mean $[5 \%, 50 \%, 95 \%]$ points from the variability distribution.

We lack sufficient information from sources to properly describe most baseline specifications' uncertainty distributions; that makes it not possible to provide fully quantitative measures of uncertainty for the summaries that we provide for outputs of interest. Reporting the uncertainty about the output is done by describing the following:

- sources of uncertainty; and

- differences in risk outputs under different uncertain conditions (storage time and temperature, flock characteristics, prevalence characteristics, egg meal and recipe allocation and egg pooling characteristics, risk mitigation practices.

\subsubsection{Baseline conditions}

\section{S. Enteritidis contaminated egg prevalence}

S. Enteritidis contaminated egg prevalence (egg prevalence) varies, as among-flock prevalence, egg within positive flock prevalence (among positive flocks) and the hens in positive flocks vary among years, or within years (Table 3).

The egg prevalence distribution has mean 1.7 per million table eggs, $5 \%$ point 4 per 10 million eggs and $95 \%$ point 3.1 per million eggs.

\section{$S$. Enteritidis levels in contaminated eggs at preparation}

Before cooking, there is a range of levels of $S$. Enteritidis among contaminated eggs due to the different times and temperatures of storage resulting in different $S$. Enteritidis growth scenarios. The initial level of $S$. 
Enteritidis at lay is $1.75 \log _{10} \mathrm{cfu}$ per egg at $50 \%$ median (1.18 and $2.23 \log _{10} \mathrm{cfu}$ at $5 \%$ and $95 \%$, respectively). S. Enteritidis can reach the maximum density of $10^{10} \mathrm{cfu}$ per egg given sufficient time at a growth permissive temperature. In contaminated eggs in which S. Enteritidis grows following 100\% yolk membrane breakdown, the levels per egg are $1.77 \log _{10} \mathrm{cfu}$ at $50 \%$ median (and 1.19 and $2.33 \log _{10}$ cfu at 5\% and 95\%, respectively).

Table 4 describes how often the frequency of 3 recognizable growth conditions -no growth; growth to $<10^{10} \mathrm{cfu}$ per egg; and growth to $10^{10} \mathrm{cfu}$ per eggvaries among contaminated eggs, given the same set of egg storage and handling conditions. Baseline conditions do not set different storage and handling distributions for eggs consumed in home and FSI settings.

While $6.8 \%$ of $S$. Enteritidis contaminated eggs experience $100 \%$ yolk membrane breakdown before consumption (Table 11, RH column), it happens most often $(85 \%$ of time that yolk membrane breakdown occurs) during consumer storage. That fewer $S$. Enteritidis contaminated eggs (4.5\%) experience growth following yolk membrane breakdown (Table 4) is attributable to

- consumer storage at temperatures below minimum growth temperature; and,

- short enough storage time, at refrigerator temperatures, that $100 \%$ yolk membrane breakdown does not occur.

At baseline specifications, approximately 33\% of consumer refrigerator temperatures and all room temperature storage ( $1 \%$ of cases) exceed minimum

\begin{tabular}{|l|l|}
\hline Prevalence characteristic & Summary statistics \\
\hline SE-flock prevalence & $0.53 \%[.28, .51, .84] \%$ \\
\hline $\begin{array}{l}\text { SE-egg within SE-flock } \\
\text { prevalence }\end{array}$ & $0.31 \%[.00074, .13,1.2] \%$ \\
\hline SE-egg prevalence & $\begin{array}{l}1.7 \times 10^{-6} \quad\left[4.0 \times 10^{-7}, 1.7 \times 10^{-6},\right. \\
\left.3.1 \times 10^{-6}\right]\end{array}$ \\
\hline
\end{tabular}

Table 3. Baseline prevalence of $S$. Enteritidis among flocks, $S$. Enteritidis-contaminated eggs from $S$. Enteritidis-infected flocks, and eggs contaminated with $S$. Enteritidis. Summary statistics (mean $[5 \%, 50 \%, 95 \%]$ points) from distributions.

\begin{tabular}{|c|c|c|}
\hline No growth & $\begin{array}{c}\text { Growth, to }<10^{10} \mathrm{cfu} \\
\text { egg }^{-1}\end{array}$ & $\begin{array}{c}\text { Growth, to } 10^{10} \mathrm{cfu} \\
\text { egg }^{-1}\end{array}$ \\
\hline $95.5[92.9,97.6]$ & $3.9[2.0,6.4]$ & $.57[.042,1.6]$ \\
\hline
\end{tabular}

Table 4. Frequency (\%) of growth conditions for S. Enteritidis contaminated eggs. Entries are a point estimate, $5 \%$ and $95 \%$ points from its uncertainty distribution (by simulation). growth temperature for $S$. Enteritidis. Final $S$. Enteritidis populations reach the maximum density in approximately $.6 \%$ of all contaminated eggs, approximately $13 \%$ of contaminated eggs that experience $100 \%$ yolk membrane breakdown.

\section{S. Enteritidis in serving}

Contaminated serving

The $S$. Enteritidis in a contaminated serving at random, from a contaminated egg at random, when the serving contains 1 or more $S$. Enteritidis (Table 5ab, $3^{\text {rd }}$ column, $S E$ ingested $\mid S E>0$ ) varies with:

- growth condition;

○ S. Enteritidis levels increase from initial contamination levels at lay only when $100 \%$ yolk membrane breakdown occurs (Table 4); and,

o $S$. Enteritidis levels in a only small fraction of contaminated eggs reach maximum population densities;

- susceptible and normal population;

o baseline specifications attribute small differences in normal and susceptible individuals' preferences for boiled eggs styles soft-boiled, poached, hard-boiled; otherwise, baseline conditions do not distinguish differences in the distributions of cooking reduction that susceptible individuals and normal individuals apply to an egg prepared in the same style type (data not shown);

- egg meal and egg recipe type (data not shown);

$\circ$ baseline specifications attribute different $\log _{10}$ reductions in $S$. Enteritidis populations to different styles of cooking egg meals and to different styles of cooking egg recipes;

o baseline specifications attribute different pooling characteristics --frequency of pooled egg dishes, number of servings from the egg dish - to different egg meal and egg recipe types;

- site (data not shown);

o baseline specifications attribute small differences in normal and susceptible individuals' preferences for boiled eggs styles soft-boiled, poached, hard-boiled;

o baseline specifications attribute substantial differences in the egg pooling characteristics associated with some egg meals and egg recipes, sharing the $S$. Enteritidis that survive cooking among more (FSI settings) or fewer (Home settings) for some egg meals and egg recipes. 


\begin{tabular}{|c|c|c|c|c|c|}
\hline \multicolumn{2}{|c|}{ Meal or recipe } & $\begin{array}{l}\text { Growth } \\
\text { conditions }\end{array}$ & $\begin{array}{l}S E \text { ingested } \mid S E>0, \\
\text { among } S E \text {-servings }\end{array}$ & $\begin{array}{l}\operatorname{Pr}\{S E>0 \mid S E-e g g\} \\
\text { among servings from } S E-e g g\end{array}$ & $\begin{array}{l}\operatorname{Pr}\{S E>0 \mid \text { egg }\} \\
\text { among eggs }\end{array}$ \\
\hline \multirow{3}{*}{\multicolumn{2}{|c|}{ Fried }} & No growth & $1.1[1,1,1]$ & $.062\left[6.3 \times 10^{-5}, 5.4 \times 10^{-3}, .37\right]$ & $1.1 \times 10^{-7}\left[7.8 \times 10^{-11}, 7.8 \times 10^{-9}, 5.9 \times 10^{-7}\right]$ \\
\hline & & Some growth & $7.1 \times 10^{4}[1,1,2]$ & $.083\left[6.6 \times 10^{-5}, 6.1 \times 10^{-3}, .53\right]$ & $1.4 \times 10^{-7}\left[8.2 \times 10^{-11}, 8.9 \times 10^{-9}, 8.6 \times 10^{-7}\right]$ \\
\hline & & $\begin{array}{l}\text { Growth to } \\
\text { saturation } \\
\end{array}$ & $\begin{array}{c}1.4 \times 10^{7}\left[1.4 \times 10^{4}, 1.0 \times 10^{6},\right. \\
\left.7.3 \times 10^{7}\right]\end{array}$ & $1[1,1,1]$ & $1.7 \times 10^{-6}\left[4.0 \times 10^{-7}, 1.7 \times 10^{-6}, 3.1 \times 10^{-6}\right]$ \\
\hline \multirow{3}{*}{\multicolumn{2}{|c|}{ Scrambled }} & No growth & $1.0[1,1,1]$ & $\begin{array}{c}1.7 \times 10^{-3}\left[1.3 \times 10^{-6}, 1.6 \times 10^{-4},\right. \\
\left.8.1 \times 10^{-3}\right]\end{array}$ & $\begin{array}{c}2.9 \times 10^{-9}\left[1.7 \times 10^{-12}, 2.4 \times 10^{-10},\right. \\
\left.1.4 \times 10^{-8}\right]\end{array}$ \\
\hline & & Some growth & $1.4 \times 10^{3}[1,1,1]$ & $.016\left[1.4 \times 10^{-6}, 1.8 \times 10^{-4}, .01\right]$ & $\begin{array}{c}2.8 \times 10^{-8}\left[1.8 \times 10^{-12}, 2.7 \times 10^{-10},\right. \\
\left.2.3 \times 10^{-8}\right]\end{array}$ \\
\hline & & $\begin{array}{l}\text { Growth to } \\
\text { saturation }\end{array}$ & $2.8 \times 10^{5}\left[275,3.0 \times 10^{4}, 1.3 \times 10^{6}\right]$ & $1[1,1,1]$ & $1.7 \times 10^{-6}\left[4.0 \times 10^{-7}, 1.7 \times 10^{-6}, 3.1 \times 10^{-6}\right]$ \\
\hline \multirow{6}{*}{ Boiled } & \multirow{3}{*}{ Normal } & No growth & $3.6[1,1,15]$ & $.44\left[2.6 \times 10^{-7}, .33,1\right]$ & $7.6 \times 10^{-7}\left[3.4 \times 10^{-13}, 3.9 \times 10^{-7}, 2.5 \times 10^{-6}\right]$ \\
\hline & & Some growth & $2.3 \times 10^{6}[1,1,21]$ & $.46\left[2.7 \times 10^{-7}, .36,1\right]$ & $7.8 \times 10^{-7}\left[3.5 \times 10^{-13}, 4.2 \times 10^{-7}, 2.6 \times 10^{-6}\right]$ \\
\hline & & $\begin{array}{l}\text { Growth to } \\
\text { saturation }\end{array}$ & $4.2 \times 10^{8}\left[54,7.7 \times 10^{7}, 2.0 \times 10^{9}\right]$ & $1[1,1,1]$ & $1.7 \times 10^{-6}\left[4.0 \times 10^{-7}, 1.7 \times 10^{-6}, 3.1 \times 10^{-6}\right]$ \\
\hline & \multirow{3}{*}{ Susceptible } & No growth & $3.1[1,1,14]$ & $.37\left[1.8 \times 10^{-7}, .12,1\right]$ & $6.3 \times 10^{-7}\left[2.2 \times 10^{-13}, 1.6 \times 10^{-7}, 2.4 \times 10^{-6}\right]$ \\
\hline & & Some growth & $1.9 \times 10^{6}[1,1,19]$ & $.38\left[1.8 \times 10^{-7}, .14,1\right]$ & $6.5 \times 10^{-7}\left[2.3 \times 10^{-13}, 1.7 \times 10^{-7}, 2.4 \times 10^{-6}\right]$ \\
\hline & & $\begin{array}{l}\text { Growth to } \\
\text { saturation }\end{array}$ & $3.5 \times 10^{8}\left[43,2.6 \times 10^{7}, 1.9 \times 10^{9}\right]$ & $1[1,1,1]$ & $1.7 \times 10^{-6}\left[4.0 \times 10^{-7}, 1.7 \times 10^{-6}, 3.1 \times 10^{-6}\right]$ \\
\hline \multirow{3}{*}{\multicolumn{2}{|c|}{ Recipe, raw }} & No growth & $2.1[1,1,5]$ & $.66[.25, .69, .98]$ & $1.1 \times 10^{-6}\left[2.0 \times 10^{-7}, 1.0 \times 10^{-6}, 2.4 \times 10^{-6}\right]$ \\
\hline & & Some growth & $1.9 \times 10^{6}[1,2,6]$ & $.67[.26, .70, .99]$ & $1.2 \times 10^{-6}\left[2.1 \times 10^{-7}, 1.0 \times 10^{-6}, 2.5 \times 10^{-6}\right]$ \\
\hline & & $\begin{array}{l}\text { Growth to } \\
\text { saturation }\end{array}$ & $\begin{array}{c}2.7 \times 10^{8}\left[1.5 \times 10^{8}, 2.0 \times 10^{8},\right. \\
\left.2.9 \times 10^{8}\right]\end{array}$ & $1[1,1,1]$ & $1.7 \times 10^{-6}\left[4.0 \times 10^{-7}, 1.7 \times 10^{-6}, 3.1 \times 10^{-6}\right]$ \\
\hline \multirow{3}{*}{\multicolumn{2}{|c|}{ Recipe, lightly cooked }} & No growth & $1.0[1,1,1]$ & $.019\left[5.7 \times 10^{-4}, 7.3 \times 10^{-3}, .081\right]$ & $3.3 \times 10^{-8}\left[6.3 \times 10^{-10}, 1.1 \times 10^{-8}, 1.4 \times 10^{-7}\right]$ \\
\hline & & Some growth & $1.9 \times 10^{4}[1,1,1]$ & $.043\left[5.9 \times 10^{-4}, 8.0 \times 10^{-3}, .13\right]$ & $7.4 \times 10^{-8}\left[6.5 \times 10^{-10}, 1.2 \times 10^{-8}, 2.2 \times 10^{-7}\right]$ \\
\hline & & $\begin{array}{l}\text { Growth to } \\
\text { saturation } \\
\end{array}$ & $\begin{array}{c}3.5 \times 10^{6}\left[1.5 \times 10^{5}, 1.5 \times 10^{6},\right. \\
\left.9.9 \times 10^{6}\right]\end{array}$ & $1[1,1,1]$ & $1.7 \times 10^{-6}\left[4.0 \times 10^{-7}, 1.7 \times 10^{-6}, 3.1 \times 10^{-6}\right]$ \\
\hline \multirow{3}{*}{\multicolumn{2}{|c|}{ Recipe, well cooked }} & No growth & $1.0[1,1,1]$ & $\begin{array}{c}5.6 \times 10^{-8}\left[8.2 \times 10^{-14}, 1.1 \times 10^{-10},\right. \\
\left.1.6 \times 10^{-7}\right]\end{array}$ & $\begin{array}{c}9.4 \times 10^{-14}\left[1.1 \times 10^{-19}, 1.7 \times 10^{-16},\right. \\
\left.2.4 \times 10^{-13}\right]\end{array}$ \\
\hline & & Some growth & $1.1[1,1,1]$ & $\begin{array}{c}1.9 \times 10^{-3}\left[8.8 \times 10^{-14}, 1.3 \times 10^{-10},\right. \\
\left.2.8 \times 10^{-7}\right]\end{array}$ & $\begin{array}{c}3.5 \times 10^{-9}\left[1.2 \times 10^{-19}, 2.0 \times 10^{-16},\right. \\
\left.4.4 \times 10^{-13}\right]\end{array}$ \\
\hline & & $\begin{array}{l}\text { Growth to } \\
\text { saturation }\end{array}$ & $8.8[1,1,26]$ & $.25\left[1.6 \times 10^{-5}, .016,1\right]$ & $4.3 \times 10^{-7}\left[2.1 \times 10^{-11}, 2.5 \times 10^{-8}, 2.2 \times 10^{-6}\right]$ \\
\hline
\end{tabular}

Table 5a. S. Enteritidis levels in serving, FSI settings. Summary statistics (mean [5\%, 50\%, 95\%] points) from distributions. 


\begin{tabular}{|c|c|c|c|c|c|}
\hline \multicolumn{2}{|c|}{ Meal or recipe } & $\begin{array}{c}\text { Growth } \\
\text { conditions }\end{array}$ & $\begin{array}{l}S E \text { ingested } \mid S E>0, \\
\text { among } S E \text {-servings }\end{array}$ & $\begin{array}{c}\operatorname{Pr}\{S E>0 \mid S E-e g g\} \\
\text { among servings from } S E-e g g\end{array}$ & $\begin{array}{c}\operatorname{Pr}\{S E>0 \mid \text { egg }\} \\
\text { among eggs }\end{array}$ \\
\hline \multirow{3}{*}{\multicolumn{2}{|c|}{ Fried }} & No growth & $1.1[1,1,1]$ & $.062\left[6.3 \times 10^{-5}, 5.4 \times 10^{-3}, .37\right]$ & $\begin{array}{c}1.1 \times 10^{-7}\left[7.8 \times 10^{-11}, 7.8 \times 10^{-9},\right. \\
\left.5.9 \times 10^{-7}\right]\end{array}$ \\
\hline & & Some growth & $7.1 \times 10^{4}[1,1,2]$ & $.083\left[6.6 \times 10^{-5}, 6.1 \times 10^{-3}, .53\right]$ & $\begin{array}{c}1.4 \times 10^{-7}\left[8.2 \times 10^{-11}, 8.9 \times 10^{-9},\right. \\
\left.8.6 \times 10^{-7}\right]\end{array}$ \\
\hline & & $\begin{array}{l}\text { Growth to } \\
\text { saturation }\end{array}$ & $\begin{array}{c}1.4 \times 10^{7}\left[1.4 \times 10^{4}, 1.0 \times 10^{6},\right. \\
\left.7.3 \times 10^{7}\right]\end{array}$ & $1[1,1,1]$ & $\begin{array}{c}1.7 \times 10^{-6}\left[4.0 \times 10^{-7}, 1.7 \times 10^{-6},\right. \\
\left.3.1 \times 10^{-6}\right]\end{array}$ \\
\hline \multirow{3}{*}{\multicolumn{2}{|c|}{ Scrambled }} & No growth & $1.0[1,1,1]$ & $\begin{array}{c}2.2 \times 10^{-3}\left[1.8 \times 10^{-5}, 4.1 \times 10^{-4},\right. \\
.010]\end{array}$ & $\begin{array}{c}3.7 \times 10^{-9}\left[2.0 \times 10^{-11}, 6.1 \times 10^{-10},\right. \\
\left.1.7 \times 10^{-8}\right]\end{array}$ \\
\hline & & Some growth & $1.4 \times 10^{3}[1,1,1]$ & $.018\left[1.8 \times 10^{-5}, 4.6 \times 10^{-4}, .016\right]$ & $\begin{array}{c}3.2 \times 10^{-8}\left[2.1 \times 10^{-11}, 6.7 \times 10^{-10},\right. \\
\left.3.0 \times 10^{-8}\right]\end{array}$ \\
\hline & & $\begin{array}{l}\text { Growth to } \\
\text { saturation }\end{array}$ & $\begin{array}{c}2.8 \times 10^{5}\left[275,3.0 \times 10^{4},\right. \\
\left.1.3 \times 10^{6}\right] \\
\end{array}$ & $1[1,1,1]$ & $\begin{array}{c}1.7 \times 10^{-6}\left[4.0 \times 10^{-7}, 1.7 \times 10^{-6},\right. \\
\left.3.1 \times 10^{-6}\right]\end{array}$ \\
\hline \multirow{6}{*}{ Boiled } & \multirow{3}{*}{ Normal } & No growth & $3.6[1,1,15]$ & $.34\left[1.6 \times 10^{-7}, .067,1\right]$ & $\begin{array}{c}5.9 \times 10^{-7}\left[2.0 \times 10^{-13}, 8.4 \times 10^{-8},\right. \\
\left.2.4 \times 10^{-6}\right]\end{array}$ \\
\hline & & Some growth & $2.3 \times 10^{6}[1,1,21]$ & $.36\left[1.7 \times 10^{-7}, .078,1\right]$ & $\begin{array}{c}6.1 \times 10^{-7}\left[2.0 \times 10^{-13}, 9.9 \times 10^{-8},\right. \\
\left.2.4 \times 10^{-6}\right]\end{array}$ \\
\hline & & $\begin{array}{l}\text { Growth to } \\
\text { saturation }\end{array}$ & $4.2 \times 10^{8}\left[54,7.7 \times 10^{7}, 2.0 \times 10^{9}\right]$ & $1[1,1,1]$ & $\begin{array}{c}1.7 \times 10^{-6}\left[4.0 \times 10^{-7}, 1.7 \times 10^{-6},\right. \\
\left.3.1 \times 10^{-6}\right]\end{array}$ \\
\hline & \multirow{3}{*}{ Susceptible } & No growth & $3.1[1,1,14]$ & $.34\left[1.6 \times 10^{-7}, .050,1\right]$ & $\begin{array}{c}5.7 \times 10^{-7}\left[2.0 \times 10^{-13}, 6.2 \times 10^{-8},\right. \\
\left.2.4 \times 10^{-6}\right]\end{array}$ \\
\hline & & Some growth & $1.9 \times 10^{6}[1,1,19]$ & $.35\left[1.6 \times 10^{-7}, .060,1\right]$ & $\begin{array}{c}5.9 \times 10^{-7}\left[2.0 \times 10^{-13}, 7.6 \times 10^{-8},\right. \\
\left.2.4 \times 10^{-6}\right]\end{array}$ \\
\hline & & $\begin{array}{l}\text { Growth to } \\
\text { saturation }\end{array}$ & $3.5 \times 10^{8}\left[43,2.6 \times 10^{7}, 1.9 \times 10^{9}\right]$ & $1[1,1,1]$ & $\begin{array}{c}1.7 \times 10^{-6}\left[4.0 \times 10^{-7}, 1.7 \times 10^{-6},\right. \\
\left.3.1 \times 10^{-6}\right]\end{array}$ \\
\hline \multirow{3}{*}{\multicolumn{2}{|c|}{ Recipe, raw }} & No growth & $2.1[1,1,5]$ & $.999[.999,1,1]$ & $\begin{array}{c}1.7 \times 10^{-6}\left[4.0 \times 10^{-7}, 1.7 \times 10^{-6},\right. \\
\left.3.1 \times 10^{-6}\right]\end{array}$ \\
\hline & & Some growth & $1.9 \times 10^{6}[1,2,6]$ & $.999[1,1,1]$ & $\begin{array}{c}1.7 \times 10^{-6}\left[4.0 \times 10^{-7}, 1.7 \times 10^{-6},\right. \\
\left.3.1 \times 10^{-6}\right]\end{array}$ \\
\hline & & $\begin{array}{l}\text { Growth to } \\
\text { saturation }\end{array}$ & $\begin{array}{c}2.7 \times 10^{8}\left[1.5 \times 10^{8}, 2.0 \times 10^{8},\right. \\
\left.2.9 \times 10^{8}\right]\end{array}$ & $1[1,1,1]$ & $\begin{array}{c}1.7 \times 10^{-6}\left[4.0 \times 10^{-7}, 1.7 \times 10^{-6},\right. \\
\left.3.1 \times 10^{-6}\right]\end{array}$ \\
\hline \multirow{3}{*}{\multicolumn{2}{|c|}{ Recipe, lightly cooked }} & No growth & $1.0[1,1,1]$ & $.25[.012, .15, .87]$ & $\begin{array}{c}4.3 \times 10^{-7}\left[1.3 \times 10^{-8}, 2.2 \times 10^{-7},\right. \\
\left.1.6 \times 10^{-6}\right]\end{array}$ \\
\hline & & Some growth & $1.9 \times 10^{4}[1,1,1]$ & $.27[.012, .16, .96]$ & $\begin{array}{c}4.7 \times 10^{-7}\left[1.3 \times 10^{-8}, 2.3 \times 10^{-7},\right. \\
\left.1.8 \times 10^{-6}\right]\end{array}$ \\
\hline & & $\begin{array}{l}\text { Growth to } \\
\text { saturation }\end{array}$ & $\begin{array}{c}3.5 \times 10^{6}\left[1.5 \times 10^{5}, 1.5 \times 10^{6},\right. \\
\left.9.9 \times 10^{6}\right]\end{array}$ & $1[1,1,1]$ & $\begin{array}{c}1.7 \times 10^{-6}\left[4.0 \times 10^{-7}, 1.7 \times 10^{-6},\right. \\
\left.3.1 \times 10^{-6}\right]\end{array}$ \\
\hline \multirow{3}{*}{\multicolumn{2}{|c|}{ Recipe, well cooked }} & No growth & $1.0[1,1,1]$ & $\begin{array}{c}1.1 \times 10^{-6}\left[1.8 \times 10^{-12}, 2.3 \times 10^{-9},\right. \\
\left.3.3 \times 10^{-6}\right]\end{array}$ & $\begin{array}{c}1.9 \times 10^{-12}\left[2.2 \times 10^{-18}, 3.4 \times 10^{-15},\right. \\
\left.5.0 \times 10^{-12}\right]\end{array}$ \\
\hline & & Some growth & $1.1[1,1,1]$ & $\begin{array}{c}3.9 \times 10^{-3}\left[1.9 \times 10^{-12}, 2.7 \times 10^{-9},\right. \\
\left.5.8 \times 10^{-6}\right]\end{array}$ & $\begin{array}{c}6.7 \times 10^{-9}\left[2.4 \times 10^{-18}, 4.1 \times 10^{-15},\right. \\
\left.9.2 \times 10^{-12}\right]\end{array}$ \\
\hline & & $\begin{array}{l}\text { Growth to } \\
\text { saturation }\end{array}$ & $8.8[1,1,26]$ & $.48\left[3.2 \times 10^{-4}, .31,1\right]$ & $\begin{array}{c}8.1 \times 10^{-7}\left[4.2 \times 10^{-10}, 3.9 \times 10^{-7},\right. \\
\left.2.6 \times 10^{-6}\right]\end{array}$ \\
\hline
\end{tabular}

Table 5b. S. Enteritidis levels in serving, Home settings. Summary statistics (mean [5\%, 50\%, 95\%] points) from distributions. 


\begin{tabular}{|c|c|c|c|c|c|c|c|}
\hline \multirow{2}{*}{$\begin{array}{l}\text { Population } \\
\text { group }\end{array}$} & \multirow[b]{2}{*}{ Meal or recipe } & \multicolumn{3}{|c|}{ Food Service and Institution settings } & \multicolumn{3}{|c|}{ Home settings } \\
\hline & & No growth & $\begin{array}{c}\text { Growth, not to } \\
\text { saturation }\end{array}$ & $\begin{array}{l}\text { Growth to } \\
\text { saturation }\end{array}$ & No growth & \begin{tabular}{|c|}
$\begin{array}{c}\text { Growth, not to } \\
\text { saturation }\end{array}$ \\
\end{tabular} & $\begin{array}{l}\text { Growth to } \\
\text { saturation }\end{array}$ \\
\hline \multirow{6}{*}{ Normal } & Fried & 27 & 1.2 & .17 & 32 & 1.4 & .20 \\
\hline & Scrambled & 11 & .47 & .07 & 14 & .59 & .09 \\
\hline & Boiled & 17 & .74 & .11 & 20 & .87 & .13 \\
\hline & Recipe, raw & .043 & .0018 & .00027 & .041 & .0018 & .00026 \\
\hline & Recipe, lightly cooked & 5.6 & .24 & .035 & 3.7 & .16 & .023 \\
\hline & Recipe, well cooked & 35 & 1.5 & .22 & 26 & 1.1 & .16 \\
\hline \multirow{6}{*}{ Susceptible } & Fried & 17 & .76 & .11 & 29 & 1.2 & .18 \\
\hline & Scrambled & 17 & .75 & .11 & 15 & .65 & .094 \\
\hline & Boiled & 18 & .78 & .11 & 20 & .86 & .12 \\
\hline & Recipe, raw & .017 & .00074 & .00011 & .080 & .0035 & .00050 \\
\hline & Recipe, lightly cooked & 4.6 & .20 & .029 & 4.1 & .18 & .026 \\
\hline & Recipe, well cooked & 38 & 1.6 & .24 & 28 & 1.2 & .17 \\
\hline
\end{tabular}

Table 6. Frequencies (\%) of $S$. Enteritidis growth scenarios among table eggs used in egg meals or recipes at food service and institution or home settings.

Regardless of the egg meal or egg recipe type, $95 \%$ of the contaminated servings contain fewer than 20 $S$. Enteritidis when the contaminated egg experienced no growth or only moderate growth from initial contamination levels. When the contaminated egg experienced growth to maximum levels, a serving at random frequently has a large number of $S$. Enteritidis, except for well-cooked egg recipes.

\section{Serving from contaminated egg}

The $S$. Enteritidis in a serving from a contaminated egg at random varies with:

- growth condition (Table 4);

- susceptible and normal population (boiled egg preferences);

- egg meal and egg recipe type (cooking reduction, pooling practices);

- $\quad$ site (egg meal and egg recipe preferences; pooling)

and includes some cases for which the $S$. Enteritidis ingested is exactly 0 (Table $5 \mathrm{ab}, 4^{\text {th }}$ column, $\operatorname{Pr}\{S E>0 \mid S E-$ egg\}) and some cases for which the $S$. Enteritidis ingested is 1 or more, with distribution shown in Table $5 \mathrm{ab}, 3^{\text {rd }}$ column. The distribution for the number of $S$. Enteritidis consumed in a serving from an egg meal or egg recipe at random made from a contaminated egg (Table 5ab, $4^{\text {th }}$ column) includes a "spike", small or large, of servings with exactly $0 \mathrm{~S}$. Enteritidis, even in the case of eggs that experience growth to maximum $S$. Enteritidis levels used in a well-cooked egg recipe. In most cases that Table 5ab describe, however, servings from contaminated eggs that experience growth to maximum levels include at least some $S$. Enteritidis. The $S$. Enteritidis level, though, varies with egg meal and egg recipe type, with probability as small as $6 \times 10^{-8}$ (Table $5 \mathrm{a}, 4^{\text {th }}$ column, recipe well-cooked, distribution mean) or $1 \times 10^{-6}$ (Table $5 \mathrm{~b}, 4^{\text {th }}$ column, recipe well-cooked, distribution mean) to probability 1 (Table $5 b, 4^{\text {th }}$ column, recipe raw, growth to saturation) or nearly 1 (Table $5 b, 4^{\text {th }}$ column, recipe raw, growth not to saturation).

\section{Serving from egg, at random}

A final component of the exposure distribution is the fraction of egg servings that contain 1 or more S. Enteritidis (Table 5ab, right hand column, $\operatorname{Pr}\{S E>0 \mid$ egg $\}$ ). The $S$. Enteritidis in a serving from an egg at random varies, as noted above, with growth condition, susceptible and normal population, egg meal and egg recipe type, site, and also with egg prevalence.

There are two ways that a serving from an egg at random can have no $S$. Enteritidis:

- all servings from single eggs or pools of eggs, none of which is contaminated, since we ignore crosscontamination; Table 3's SE-egg prevalence row describes how the frequency of $S$. Enteritidis contaminated eggs varies; and,

- some servings from single eggs or pools of eggs, one or more of which is contaminated, more when the level of $S$. Enteritidis in the single egg or pool is low, fewer when the level of $S$. Enteritidis in the single egg or pool is high.

\section{Pathway frequencies}

Table 6 reports the frequency of each pathway in Table 5ab's $1^{\text {st }}$ and $2^{\text {nd }}$ columns for FSI settings and for Home settings. Pathway frequencies for population groups and meals or recipes are part of the baseline specification (data not shown); pathway frequencies for growth conditions are derived by simulation from the storage and handling conditions, the rate at which yolk membrane breakdown occurs and the rate of growth of $S$. Enteritidis in a contaminated egg after $100 \%$ yolk membrane breakdown occurs. 


\subsubsection{Special cases and sensitivity}

\section{S. Enteritidis egg prevalence}

The influential variables for contaminated egg prevalence and their order of importance remain the same as in the previous risk assessment [1]: flock prevalence, sizes of $S$. Enteritidis positive flocks, egg-within flock prevalence.

Different flock prevalence (Table $7,2^{\text {nd }}$ column) for the same within-flock egg prevalence, whether the number of hens in $S$. Enteritidis positive flocks is fixed at a particular total size or not (Table 7, $3^{\text {rd }}$ column) and different withinflock egg prevalence yield different $S$. Enteritidis egg prevalence (Table 7, RH column; Table 9, RH column).

The level of $S$. Enteritidis contaminated egg prevalence, such as at the mean and median, increase when:

- flock prevalence increases (Table 7, among rows with different flock prevalence);

- within-flock egg prevalence increases (Table 9, among rows with different within-flock egg prevalence); and,

- the number of hens in an infected flock increases (Table 8, among rows)

and the level of $S$. Enteritidis egg prevalence, such as at the median and mean decrease when:

- flock prevalence decreases (Table 7, among rows with different flock prevalence);

- within-flock egg prevalence decreases (Table 9, among rows with different within-flock egg prevalence); and,

- the number of hens in an infected flock decreases (Table 8, among rows)

Smallest S. Enteritidis contaminated egg prevalence occurs when flock prevalence is smallest, within flock prevalence is smallest and the number of hens in positive flocks is smallest.

Variability in the contaminated egg prevalence per $10^{6}$ eggs increases when:

- flock prevalence is larger (Table $7,1^{\text {st }}$ row versus last row);
- flock prevalence specification covers variability [1], [39], [54] and is more variable, itself [1] than when flock prevalence refers to a single, fixed value from that flock prevalence distribution (Table $7,3^{\text {rd }}$ row versus last row; Table $7,5^{\text {th }}$ row versus last row);

- total size of $S$. Enteritidis positive flocks varies (Table 7, rows containing ${ }^{* *}$ in $3^{\text {rd }}$ column; Table 8 versus Table $7,2^{\text {nd }}$ row) or is more variable, itself; and,

- egg within flock prevalence is more variable (Table 9, $3^{\text {rd }}$ column).

Additional comments describe how egg prevalence changes under flock prevalence and egg within flock prevalence characteristics that are not described in the microbiological and animal husbandry literature.

\section{S. Enteritidis flock prevalence}

Baseline specifications for flock prevalence use the two reported flock prevalences [39], [54] from Table 1 to describe how flock prevalence varies among, nominally, years. Other flock prevalence descriptions (Table 7 and Figure 4) come from:

- Paoli representation [1] of data from Poppe et al. [48];

- the environmental test results from CEMA for a single year [39]; and,

- the environmental test results from CEMA for a single year [54].

The CEMA point flock prevalence, $.28 \%$, for the flocks tested [54], is at the $5 \%$ point of the baseline flock prevalence distribution; the CEMA point flock prevalence, $.77 \%$, for the flocks tested [39], is at the $91 \%$ point of the baseline flock prevalence distribution; the mean value, $2.9 \%$, from the flock prevalence distribution in Paoli [1], is at a very high quantile of the baseline flock prevalence distribution.

Additional information from Table 2's flock environment testing results -the total number of hens in the positive flocks - fixes what acts as a single observation on egg prevalence in a nominal year within the egg prevalence distributions, subject to unknown test sensitivity.

\begin{tabular}{|c|c|c|c|c|}
\hline Specification source & $\begin{array}{c}\text { Flock prevalence } \\
\text { specification }\end{array}$ & Flock prevalence (\%) & $\begin{array}{l}\text { Hens in } \\
\text { SE-flocks }\end{array}$ & $\begin{array}{l}\text { SE-egg prevalence } \\
\text { per } 10^{6} \text { eggs }\end{array}$ \\
\hline Paoli (2001) [1] & $\operatorname{Beta}(9,306)$ & $2.9[1.5,2.8,4.5]$ & $* *$ & $8.9[3.5,8.8,14]$ \\
\hline \multirow{2}{*}{ CEMA (2007a) [39] } & \multirow{2}{*}{$12 / 1550$} & \multirow{2}{*}{.77} & 154664 & $3.1[2.8,3.1,3.4]$ \\
\hline & & & $* *$ & $2.4[1.0,2.4,3.8]$ \\
\hline \multirow{2}{*}{ CEMA (2007b) [54] } & \multirow{2}{*}{$4 / 1437$} & \multirow{2}{*}{.28} & 137940 & $2.1[1.8,2.1,2.5]$ \\
\hline & & & $* *$ & $.91[.19, .89,1.7]$ \\
\hline CEMA (2007ab) baseline & $\operatorname{Beta}(9.44,1766.9)$ & $.53[.28, .51, .84]$ & ** & $1.7[.40,1.7,3.1]$ \\
\hline
\end{tabular}

Table 7. Egg prevalence distribution (among years or within years) summary statistics (mean [5\%, 50\%, 95\%] points) under various flock prevalence specifications, with within-flock egg prevalence at baseline specification. Italicized row is the baseline specification. 


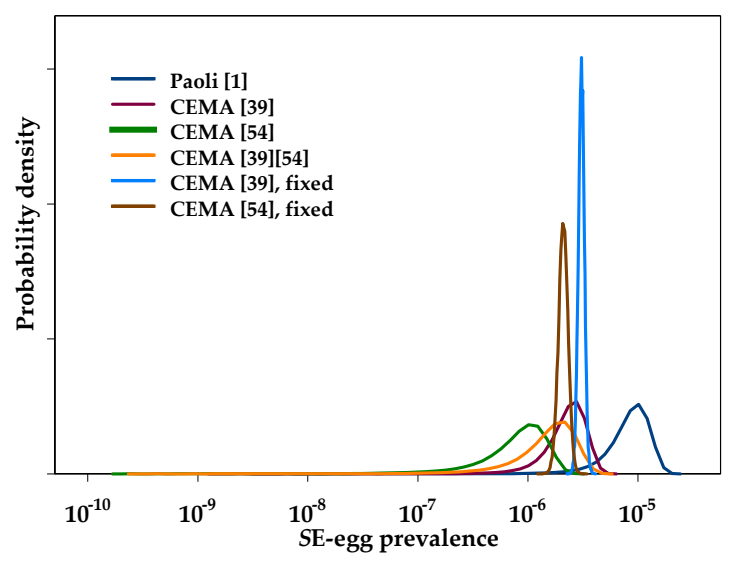

Figure 4. Prevalence of contaminated eggs under different flock prevalence specifications.

Table 7 provides a contrast in the egg prevalence within the table eggs consumed. At baseline within flock egg prevalence, contaminated egg prevalence has a distribution with

- identically 0 contaminated eggs per $10^{6}$ eggs among table eggs from not infected flocks;

- mean prevalence 8.9 contaminated eggs per $10^{6}$ eggs among table eggs from a population of flocks with mean flock prevalence 2.9\% (Paoli, 2001 baseline [1]);

- mean prevalence 3.1 contaminated eggs per $10^{6}$ eggs from table eggs from a population of flocks with mean flock prevalence $.77 \%$;

- mean prevalence 2.1 contaminated eggs per $10^{6}$ eggs from table eggs from a population of flocks with mean flock prevalence $.28 \%$;

- mean prevalence 2.4 contaminated eggs per $10^{6}$ eggs among table eggs from a population of flocks with mean flock prevalence .53\% (baseline); etc.

\section{S. Enteritidis flock size}

The effect of the number of hens in positive flocks on egg prevalence is illustrated in Table 8, for which we selected the values in the left column to correspond to quantiles of the distribution of the sum of 12 flock sizes from the nominal flock size distribution. The $5 \%$ point from the distribution of the sum of 12 flocks' sizes at random is approximately 88000 hens; the $95 \%$ point from the distribution of the sum of 12 flocks' sizes at random is approximately 225000 hens. The 12 corresponds to the number of positive flocks identified in 2005 (Table 7). For the calculation in Table 8, we simply fix flock prevalence at CEMA reported level, .77\% [39].

\begin{tabular}{|c|c|}
\hline $\begin{array}{c}\text { Hens in SE-positive } \\
\text { flocks }\end{array}$ & SE-egg prevalence $\times 10^{-6}$ \\
\hline 88000 & $1.8[1.4,1.8,2.0]$ \\
\hline 100000 & $2.0[1.6,2.0,2.3]$ \\
\hline 140000 & $2.8[2.3,2.8,3.2]$ \\
\hline 175000 & $3.5[2.9,3.5,4.0]$ \\
\hline 200000 & $4.0[3.3,4.1,4.5]$ \\
\hline 225000 & $4.5[3.7,4.6,5.1]$ \\
\hline
\end{tabular}

Table 8. Effect on $S$. Enteritidis-egg prevalence distribution (among years or within years) summary statistics (mean [5\%, $50 \%, 95 \%$ ] points) of varying the number of hens in 12 S. Enteritidis positive flocks.

Results in Table 8 illustrate, for example, how the egg prevalence changes as probability of flock infection changes across the flock population. When flock prevalence increases with flock size, then the distribution of $S$. Enteritidis infected flock sizes takes on larger values with increasing probability, the distribution of total hens in positive flocks has more density at higher values and egg prevalence takes on values more like those at the bottom of Table 8 . When flock prevalence decreases with flock size, then the distribution of $S$. Enteritidis infected flock sizes takes on smaller values with increasing probability, the distribution of total hens in positive flocks has more density at lower values and egg prevalence takes on values more like those at the top of Table 8.

The animal husbandry and microbiological literature are inconclusive as to whether flock prevalence changes with flock size.

\section{S. Enteritidis egg within flock prevalence}

Alternative descriptions for how within-flock contaminated egg prevalence varies come from

- Paoli [1] representation of data from the United States Pennsylvania Pilot Project [8][59];

- Paoli [1] representation of data from Poppe et al. [49]; and,

- a description of the variability across those data sets and lead to different egg prevalence (Table 9).

Test and divert programs, which also limit egg within flock prevalence, are treated in more detail, as a risk management option (section 6.1.2).

Table 9 also contrasts between the egg prevalence within the table eggs consumed. Egg prevalence is

- identically 0 contaminated eggs per $10^{6}$ eggs among the table eggs from not infected flocks;

- mean prevalence 3 contaminated eggs per $10^{4}$ eggs among the table eggs from infected flocks (the within-flock contaminated egg prevalence); and,

- mean prevalence 1.7 contaminated eggs per $10^{6}$ eggs among eggs, at random, ignoring flock infection status. 


\begin{tabular}{|l|c|c|}
\hline \multicolumn{1}{|c|}{ Specification Source } & $\begin{array}{c}\text { Within-flock } \\
\text { prevalence (\%) }\end{array}$ & $\begin{array}{c}\text { SE-egg } \\
\text { prevalence per } \\
10^{6} \text { eggs }\end{array}$ \\
\hline Pennsylvania Pilot Project & $\begin{array}{c}.034[.025, .034, \\
.044]\end{array}$ & $1.9[.44,1.8,3.4]$ \\
\hline Canadian & $\begin{array}{c}.012[.0021, .010, \\
.029]\end{array}$ & $.69[.13, .67,1.3]$ \\
\hline $\begin{array}{l}\text { Combined Cdn, PA prior } \\
\text { (Paoli, 2001) baseline }\end{array}$ & $\begin{array}{c}.031[.023, .031, \\
.040]\end{array}$ & $1.7[.40,1.7,3.1]$ \\
\hline
\end{tabular}

Table 9. Egg prevalence distribution (among years or within years) summary statistics (mean $[5 \%, 50 \%, 95 \%]$ points) under alternative within-flock prevalence specifications, with flock prevalence at baseline specification. Italicized row is the baseline specification.

Under the same baseline specifications for flock prevalence, egg within flock and flock size distributions, the egg prevalence distribution changes when egg within flock and flock size are related (Figure 5). For example, when large infected flocks have larger within flock prevalence and small infected flocks have smaller within flock prevalence, then the egg prevalence distribution shifts to higher values; smaller egg prevalence values occur less frequently and larger egg prevalence values occur more frequently than under the baseline specifications. When small infected flocks have larger within flock prevalence and large infected flocks have smaller within flock prevalence, then the egg prevalence distribution shifts to smaller values; smaller egg prevalence values occur more frequently and larger egg prevalence values occur less frequently than under the baseline specifications.

The animal husbandry and microbiological literature are inconclusive as to whether egg within flock prevalence changes with the size of the infected flock. If egg within flock prevalence increases with larger infected flocks, then the egg prevalence distribution has summary statistics closer to the ones at the bottom of Table 8. If egg within flock prevalence decreases with larger infected flock size, then the egg prevalence distribution shifts towards those at the top of Table 8 .

\section{Initial S. Enteritidis in egg}

Alternative specifications for $S$. Enteritidis deposition at lay [5] and growth immediately after lay [8] lead to $17 \%$ higher and $41 \%$ lower mean stable $S$. Enteritidis in albumen concentrations and to $13 \%$ higher, nominally at the mean, and $7 \%$ lower, nominally at the mean over the distribution of $S$. Enteritidis levels in an egg at point of preparation, than in the baseline specifications used here.

USDA synthesized a distribution of locations within the egg where S. Enteritidis is deposited at lay, some in the albumen far from the yolk, some in the albumen near the yolk, some in the albumen on the vitelline membrane and some (rarely) in the yolk [8]. That method of modeling the concentration of $S$. Enteritidis at lay provides a less

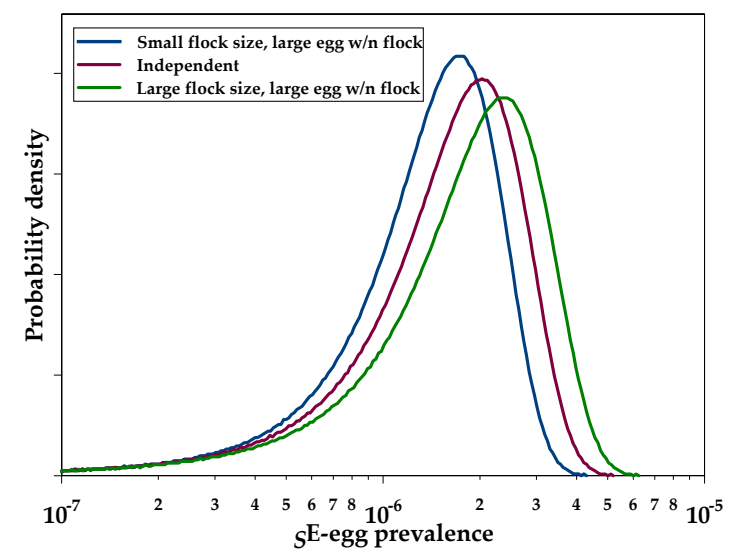

Figure 5. Prevalence of contaminated eggs under different egg within flock prevalence specifications.

conservative $S$. Enteritidis concentration in the contaminated egg than the baseline specification used here.

\section{Impact of storage and handling conditions}

Within baseline conditions

Time to $100 \%$ yolk membrane breakdown (YMB) varies from egg to egg -rates of yolk membrane breakdown at the same temperature vary from egg to egg and storage and holding temperatures vary from egg to egg. Among eggs, $100 \%$ yolk membrane breakdown seldom occurs before five days except when the egg is stored at temperatures above $25^{\circ} \mathrm{C}$ (Table $10,2^{\text {nd }}$ column; Table 10 , columns 3-6). However, even at $10^{\circ} \mathrm{C}$, the average over the distribution of percentage yolk membrane breakdown is larger than $50 \%$ after approximately 24 days (Table 10 ).

$100 \%$ yolk membrane breakdown seldom occurs among baseline egg storage and handling conditions and rarely occurs before consumer storage (Table 11, RH column). Approximately $5 \%$ of eggs have $\geq 71 \%$ membrane integrity loss by the end of the retail storage (Table 11, $4^{\text {th }}$ column), at the time of purchase by the consumer. However, under conditions where an egg has been stored or handled at high enough temperatures for long enough periods, $100 \%$ yolk membrane breakdown can occur in short periods of time if the consumer stores the egg at elevated temperatures for further lengths of time.

Little further yolk membrane breakdown occurs if consumer storage temperatures are low and $100 \%$ yolk membrane breakdown is not reached if the consumer eats the egg within a short time after purchase.

Storage and handling conditions examined in a simulation experiment showed that yolk membrane breakdown frequency is insensitive to baseline storage and handling conditions misspecification and to the more influential stage time and temperature components: 


\begin{tabular}{|c|c||c|c|c|c|c|c|c|c|}
\hline \multirow{2}{*}{$\begin{array}{c}\text { Temp. } \\
\left({ }^{\circ} \mathrm{C}\right)\end{array}$} & \multicolumn{2}{|c||}{$\begin{array}{c}\text { Days to } \\
100 \% \text { YMB }\end{array}$} & \multicolumn{3}{c|}{ Time to $100 \%$ YMB less than } & \multicolumn{4}{c|}{ Mean percentage YMB in } \\
\hline 5 & $78[44,74,125]$ & $2 \mathrm{~d}$ & $5 \mathrm{~d}$ & $10 \mathrm{~d}$ & $15 \mathrm{~d}$ & $5 \mathrm{~d}$ & $10 \mathrm{~d}$ & $15 \mathrm{~d}$ & $20 \mathrm{~d}$ \\
\hline 5 & & & & & 7 & 14 & 21 & 28 \\
\hline 10 & $49[28,47,79]$ & & & & .013 & 11 & 22 & 34 & 45 \\
\hline 15 & $31[18,30,50]$ & & & .033 & 1.6 & 18 & 36 & 53 & 71 \\
\hline 20 & $20[11,19,31]$ & & & 2.4 & 25 & 28 & 56 & 85 & 100 \\
\hline 25 & $12[7,12,20]$ & & .34 & 30 & 78 & 45 & 89 & 100 & 100 \\
\hline 30 & $7.8[4.4,7.4,12]$ & & 11 & 83 & 99 & 71 & 100 & 100 & 100 \\
\hline 45 & $2.0[1.1,1.9,3.1]$ & 59 & 100 & 100 & 100 & 100 & 100 & 100 & 100 \\
\hline
\end{tabular}

Table 10. Temperature-dependent characteristics, as they vary among eggs, of time to yolk membrane breakdown (YMB). Entries for Days to $Y M B$ are mean $[5 \%, 50 \%, 95 \%$ ] points from that distribution and ignore maximum time to $100 \%$ yolk membrane breakdown.

\begin{tabular}{|l|c|c|c|c|}
\hline \multicolumn{1}{|c|}{ Stage } & Stage duration $(\mathrm{h})$ & \% YMB in stage & $\begin{array}{c}\text { Cumulative\% YMB, } \\
\text { end stage }\end{array}$ & $\begin{array}{c}\text { Frequency of complete YMB } \\
\text { by end stage (\%) }\end{array}$ \\
\hline Barn & $8.1[1.6,7.4,20]$ & $3.7[1.9,3.5,6.1]$ & $3.7[.55,2.8,9.9]$ & 0 \\
\hline On farm & $43[3.9,40,104]$ & $6.0[3.6,5.6,9.8]$ & $9.6[2.4,8.2,21]$ & 0 \\
\hline Transport 1 & $2.7[.94,2.6,5.2]$ & $.38[.24, .36, .61]$ & $10[2.7,8.5,22]$ & 0 \\
\hline Pre-grading & $13[2.2,13,23]$ & $1.3[.95,1.2,1.8]$ & $11[3.7,10,23]$ & 0 \\
\hline Grading & $.23[.13, .23, .36]$ & $.056[.031, .053, .091]$ & $11[3.8,10,23]$ & $2.0 \times 10^{-3}$ \\
\hline Post-grading & $62[23,59,112]$ & $6.4[4.8,6.1,8.9]$ & $17[8.0,16,32]$ & $2.0 \times 10^{-3}$ \\
\hline Transport 2 & $3.5[1.3,3.5,5.7]$ & $.36[.27, .35, .50]$ & $18[8.3,16,32]$ & .022 \\
\hline Wholesale & $41[0,0,213]$ & $4.1[1.7,3.9,7.2]$ & $22[8.9,19,43]$ & .022 \\
\hline Retail deviations & $.049[0,0,0]$ & $.012[0,0, .077]$ & $22[8.9,19,43]$ & 1.1 \\
\hline Retail storage & $142[21,96,437]$ & $15[9.6,14,22]$ & $36[15,32,71]$ & 6.8 \\
\hline Consumer storage & $164[12,119,466]$ & $18[11,17,28]$ & $53[22,47,102]$ & \\
\hline
\end{tabular}

Table 11. Storage and handling stage yolk membrane breakdown characteristics' among eggs distribution summary statistics (mean $[5 \%, 50 \%, 95 \%])$.

- higher versus lower temperature settings for the most influential stages (Barn, On-farm, Retail, Consumer) and higher versus lower time settings for the most influential stages (Grade, Post-grade, Consumer) account for smaller than $5 \%$ increase (higher settings) or $5 \%$ decrease (lower settings) in the yolk membrane breakdown conditions and growth to final $S$. Enteritidis levels in a contaminated egg;

- storage time and temperature in ultimate stages (Retail, Consumer) are most influential, and are more influential still when time and temperature conditions in previous storage and handling stages lead to some yolk membrane breakdown; and,

- storage time and temperature in retail and consumer stages are more influential for the $S$. Enteritidis levels in a contaminated egg when storage and handling prior to those stages has led to yolk membrane breakdown approaching 100\%; storage time and temperature in retail and consumer stages are less influential for the $S$. Enteritidis levels in a contaminated egg when storage and handling prior to those stages has led to yolk membrane breakdown much less than $100 \%$.

Baseline specifications constrain time to $100 \%$ yolk membrane breakdown, at any temperature or any combination of stage times and temperatures, to a fixed, but uncertain, maximum length as $\operatorname{Pert}(42,50,62)$ days. Cumulative time from production to consumption exceeds the uncertain maximum time for $.91 \%$ [.36\%, $2.0 \%$ ] $\left(2 \frac{1}{2} \%\right.$ and $97 \frac{1}{2} \%$ points from uncertainty distribution, by simulation) of table eggs under baseline conditions. The uncertain maximum constrains the time to $100 \%$ yolk membrane breakdown, conservatively, but by only a small amount.

$S$. Enteritidis growth does not occur until after the yolk vitelline membrane breaks down (section 2.6) and after an additional lag time after vitelline membrane breakdown [78]. Therefore, most growth occurs during consumer storage of the eggs [1], [4], [6], [8], [78] since there is rarely sufficient time (at temperature) for yolk membrane breakdown to occur before consumer storage.

Other storage times and temperatures

Using alternative consumer refrigerator temperature information:

- at refrigerator temperatures attributed to American homes [98], 100\% yolk membrane breakdown would occur less frequently and temperatures that would permit $S$. Enteritidis growth would appear markedly less frequently than is the case in the baseline storage and handling conditions $(7 \%$ of refrigerator temperatures versus $33 \%$ of cases exceeding $8^{\circ} \mathrm{C} ; 2 \%$ versus $4.4 \%$ exceeding $10^{\circ} \mathrm{C}$ ); and,

- alternative information that indicates more frequent, higher consumer refrigerator storage temperature in several European countries [99], [102] that would 
lead to more frequent $100 \%$ yolk membrane breakdown at the consumer phase and more $S$. Enteritidis in contaminated eggs, are deemed to be not applicable to the Canadian consumer.

Using alternative information about consumer egg storage time in home settings:

- from American consumers for an individual egg [6], [8], [10] has approximately the same mean but shorter upper tail and would lead to slightly less frequent yolk membrane breakdown and less frequent $S$. Enteritidis growth in a contaminated egg before consumption; and,

- from Finnish consumers for a dozen eggs [102] has a larger mean and longer upper tail and would lead to more frequent yolk breakdown and more frequent $S$. Enteritidis growth to high levels before consumption.

The effect on risk estimates for FSI settings under different storage time specifications is examined as part of risk management options considered in section 6.2.1.

Other yolk membrane breakdown characteristics

Time at storage temperature for $100 \%$ yolk membrane breakdown in Whiting et al. [5] is nearly identical to that of this risk assessment. USDA [8] derived a distribution for the time to $100 \%$ yolk membrane breakdown as a function of storage temperature that represents longer mean time to yolk membrane breakdown than the derivation used here. Under longer mean time to yolk membrane breakdown, less $S$. Enteritidis growth occurs, in fewer storage and handling scenarios and leads to lower levels of contamination in contaminated eggs at point of consumption.

\section{Other S. Enteritidis growth characteristics}

There are only small differences in the amount of S. Enteritidis growth after $100 \%$ yolk membrane breakdown under alternative $S$. Enteritidis growth rates in Whiting et al. [5], under the alternative growth rates in USDA [8] and under the $S$. Typhimurium alternative [10], [11].

Alternatives' maximum population density specifications allow the same [5], slightly more [8] and slightly less [10] maximum population densities in a contaminated egg than does the baseline specification here.

\section{Cooking reduction}

Figure 6 illustrates the quite different cooking effect that preparing different types of egg meals and egg recipes has on the $S$. Enteritidis levels in a contaminated egg, there, for a normal individual at home settings. Cooking $\log _{10}$ reductions vary both between meal types -fried eggs, scrambled eggs, boiled eggs- and recipe types -raw, lightly cooked, well cooked- and within meal and recipe types.
The cooking reduction for boiled eggs also accounts for the quite sizable differences between soft-boiled and poached eggs and hard-boiled eggs cooking effects: its distribution of $\log _{10}$ reductions is the mixture of 2 distributions, 1 centred at approximately $8 \log _{10}$ for hardboiled eggs and the other centred at approximately $2 \log _{10}$ for soft-boiled eggs and poached eggs. Eggs consumed raw do not have a cooking reduction.

There are small differences between home and FSI settings and between normal and susceptible individuals -boiled egg and egg recipe preferences in the baseline specification vary between population groups and between sites. Given large differences in cooking reductions for different egg meal types and egg recipe types, small changes in cooking preferences would effect changes in the $S$. Enteritidis in servings from contaminated eggs. Changing the distribution of egg meal and egg recipe preferences from ones with low cooking effects to ones with high cooking effects reduces the frequency of servings with $S$. Enteritidis; removing all raw egg consumption and all lightly cooked egg recipe consumption, for example, removes two of the larger contributors of servings with low cooking effects.

\section{Egg preparation practices}

Inferences from the 24-hour recall data in a series of Federal-Provincial Nutrition Surveys conducted from 1991 to 1999 [97] inform the baseline specification about how individual preferences in preparation and consumption of eggs vary for egg meals versus egg recipes, among egg meal types and among egg recipe types among individuals within population groups and between home and FSI settings. Alternatives for preparation practices from other precedent risk assessments describe preparation preferences for other countries' populations and are not deemed appropriate for Canada.

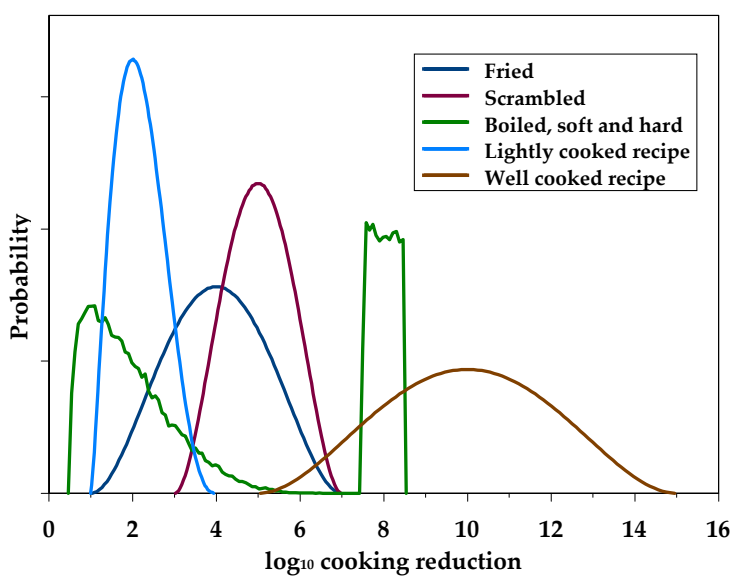

Figure 6. S. Enteritidis levels in a contaminated egg under cooking effects from different meal and recipe types. 


\begin{tabular}{|c|c|c|}
\hline Egg prevalence & \multicolumn{2}{|l|}{$1.7 \times 10^{-6}\left[4.0 \times 10^{-7}, 1.7 \times 10^{-6}, 3.1 \times 10^{-6}\right]$} \\
\hline Illnesses per contaminated egg & \multicolumn{2}{|l|}{$.014\left[5.1 \times 10^{-3}, 8.2 \times 10^{-3}, .046\right]$} \\
\hline Home settings & \multicolumn{2}{|l|}{$8.9 \times 10^{-3}\left[1.6 \times 10^{-3}, 4.0 \times 10^{-3}, .017\right]$} \\
\hline FSI settings & \multicolumn{2}{|l|}{$.029[.012, .021, .067]$} \\
\hline Pr\{illness | SE-egg \} & Normal & Susceptible \\
\hline Home settings & $7.0 \times 10^{-3}\left[6.2 \times 10^{-13}, 1.6 \times 10^{-5}, .035\right]$ & $.012\left[1.2 \times 10^{-12}, 1.8 \times 10^{-5}, .063\right]$ \\
\hline FSI settings & $7.5 \times 10^{-3}\left[2.0 \times 10^{-14}, 5.6 \times 10^{-6}, .037\right]$ & $9.2 \times 10^{-3}\left[2.5 \times 10^{-14}, 7.4 \times 10^{-7}, .055\right]$ \\
\hline Pr\{illness | egg $\}$ & Normal & Susceptible \\
\hline Home settings & $1.4 \times 10^{-8}\left[1.4 \times 10^{-18}, 2.2 \times 10^{-11}, 5.7 \times 10^{-8}\right]$ & $1.9 \times 10^{-8}\left[2.0 \times 10^{-18}, 2.7 \times 10^{-11}, 9.6 \times 10^{-8}\right]$ \\
\hline FSI settings & $1.2 \times 10^{-8}\left[2.6 \times 10^{-20}, 8.9 \times 10^{-12}, 5.5 \times 10^{-8}\right]$ & $1.6 \times 10^{-8}\left[3.7 \times 10^{-20}, 1.0 \times 10^{-12}, 8.6 \times 10^{-8}\right]$ \\
\hline Number of illnesses per year & \multicolumn{2}{|l|}{$120[20,102,280]$} \\
\hline Minor, severe, fatalities & \multicolumn{2}{|l|}{$115[20,97,268] ; 5.3[0,4,14] ; .050[0,0,0]$} \\
\hline
\end{tabular}

Table 12. Summary statistics (mean $[5 \%, 50 \%, 95 \%]$ point) of distributions at baseline conditions.

The Canadian Community Health Survey nutrition component [103] provides information from a more recent survey than the Federal-Provincial Nutrition Surveys [97] used for this work.

\section{Pooling characteristics}

Alternative pooling characteristics (data not shown) in home and FSI settings from USDA [8] set home pooling frequency at $10 \%$ of and FSI pooling frequency at $20 \%$ of the baselines used here. USDA [8] specification for home egg meal and egg recipes when eggs are pooled implicates approximately 2 times as many servings (mean value) than the baseline specification here and USDA [8] for FSI egg meal and egg recipes when eggs are pooled implicates $1 / 2$ as many servings (mean value) than the baseline specification here.

\section{Risk Characterization}

Primary outputs from the risk assessment model are distributions of the prevalence of contaminated eggs, the number of illnesses per S. Enteritidis contaminated egg and the number of illnesses that would accrue under those conditions. The outputs are distributions: they describe how the risk outputs vary over the conditions established in the baseline set of conditions.

To facilitate reporting and to describe how the risk outputs vary, we

- differentiate between the distributions for FSI and home settings; among the distributions for meal or recipe types; among the distributions for growth conditions and between the distributions for population groups; and,

- report summary statistics from the distributions that describe how the risk varies within those breakdowns

plus report some further summaries (Table 12).

The outputs refer to the baseline set of conditions established in the exposure assessment (section 4.1). For those baseline conditions, the average prevalence of contaminated eggs is 1.7 per million eggs and the average number of illnesses is 120 per year, $95 \%$ of which are minor illnesses (Table 12).

Risks vary with the following:

- flock and within-flock egg prevalence, infected flocks' total size;

- S. Enteritidis level in a contaminated egg: initial contamination and short-term growth, and storage and handling time- and temperature-dependent yolk membrane breakdown and $S$. Enteritidis growth;

- cooking reduction: with type of egg meal (fried, scrambled, boiled) and with type of recipe (raw, lightly-cooked, well-cooked), among meals or recipes of the same type, and with relative frequency of meals and recipes consumed at home settings and food service \& institution settings;

- illness from consuming $S$. Enteritidis in an egg serving: $S$. Enteritidis in one of multiple servings from a preparation from a contaminated egg and among-population groups' (normal, susceptible) response to $S$. Enteritidis consumed.

Risk outputs are subject to uncertainty from the following:

- flock prevalence, within flock prevalence, egg within hen prevalence, egg within flock prevalence, environment prevalence, and test sensitivity;

- current risk management practices directed against prevalence and how those practices are followed;

- S. Enteritidis initial contamination, and growth dynamics (yolk membrane breakdown, minimum growth temperature, growth rate and maximum population density as a function of temperature);

- cooking reduction, relative proportion of egg meals, egg recipes, meal types among egg meals and recipe types among recipes;

- multiple servings from a preparation from an $S$. Enteritidis contaminated egg; and,

- dose-response for a population of normal and a population of susceptible individuals,

none of which is fully quantifiable given the information available. 


\subsection{Baseline conditions}

\subsubsection{Basic risk results}

\section{Contaminated egg prevalence}

Contaminated egg prevalence among all table eggs varies, nominally over a year's egg production. Mean prevalence from that distribution is 1.7 contaminated eggs per million table eggs consumed; $5 \%$ of the time, egg prevalence would be $\leq 4$ contaminated eggs per 10 million table eggs consumed and $5 \%$ of the time, egg prevalence would be $\geq 3.1$ contaminated eggs per million table eggs consumed, under the same baseline conditions (Table 12).

Results in sections 4.2.1 and 4.2.2 provided also several contrasts between the egg prevalence within the table eggs consumed. Egg prevalence is

- identically 0 contaminated eggs per $10^{6}$ eggs among the table eggs from not infected flocks;

- mean prevalence 3 contaminated eggs per $10^{4}$ eggs among the table eggs from infected flocks (the within-flock contaminated egg prevalence); that prevalence holds in the table egg production from the $.53 \%$ (flock prevalence distribution mean) of flocks that are infected; and,

- mean prevalence 1.7 contaminated eggs per $10^{6}$ eggs among eggs, at random, ignoring flock infection status.

\section{Illnesses per contaminated egg}

We distinguish the number of illnesses per contaminated egg by eggs consumed in home settings and eggs consumed in FSI settings. The number of illnesses per contaminated egg in home settings varies, nominally among contaminated eggs, independently of egg prevalence. In home settings, mean number of illnesses per contaminated egg is 0.0089 , approximately 9 illnesses per 1,000 contaminated table eggs consumed. In about $5 \%$ of contaminated eggs, the number of illnesses per contaminated egg is at or higher than 17 per 1,000 contaminated eggs. The mean number of illnesses per contaminated egg in FSI settings is 29 per 1,000 contaminated eggs. For $5 \%$ of contaminated eggs, the number of illnesses per contaminated egg is $\leq 12$ per 1,000 contaminated eggs and for $5 \%$ of contaminated eggs, the number of illnesses per contaminated eggs is $\geq 67$ per 1,000 contaminated eggs.

\section{Probability of illness from a contaminated egg}

The probability of illness from consuming a serving, at random, from a contaminated egg, at random (either the whole egg or some part of an egg when the egg is pooled and shared among several servings) varies over 11 or 12 orders of magnitude. In approximately $5 \%$ of the cases, the probability of illness from a single contaminated egg is smaller than approximately $10^{-13}$ or $10^{-14}$; in approximately $5 \%$ of the cases, the probability of illness from a single contaminated egg is larger than .04 (normal individual) or .06 (susceptible individual). A contaminated serving, at random, encompasses all cases described by egg meal and egg recipe types, their cooking reductions and egg pooling characteristics. A contaminated egg, at random, covers all cases described by storage and handling, yolk membrane breakdown and growth and initial deposition at lay.

The probability of illness from consuming a serving from a contaminated egg, at random, is 1.2 times (FSI settings) and 1.7 times (home settings) higher (at mean, Table 12) for susceptible individuals than for normal individuals. Susceptible individuals have different egg consumption patterns than a normal individual; susceptible individuals have higher probability of illness from consuming the same number of $S$. Enteritidis than does a normal individual.

\section{Probability of illness from an egg at random}

The probability of illness from consuming a serving, at random, from an egg, at random, accounts for both the probability of illness from consuming a serving, at random, from a contaminated egg, at random, and the prevalence of contaminated eggs. The probability of illness from consuming a serving from an egg at random (either the whole egg or some part of an egg when the egg is pooled and shared among several servings) varies over several orders of magnitude. Average probability of illness varies from $1.2 \times 10^{-8}$ (normal individual, FSI setting) to $1.9 \times 10^{-8}$ (susceptible individual, home setting). In approximately $5 \%$ of cases, the probability of illness is smaller than $10^{-18}$ to $10^{-19}$; in approximately $5 \%$ of cases, the probability of illness is larger than approximately $10^{-7}$.

\section{Number of illnesses per year}

Under baseline conditions for $S$. Enteritidis egg prevalence, with $S$. Enteritidis levels in a contaminated egg (section 4.1-4.2), the number of S. Enteritidis-egg associated illnesses varies. The distribution has mean 120 illnesses per nominal year. In $5 \%$ of nominal years, the number of illnesses is less than 20 and in $5 \%$ of nominal years, the number of illnesses is larger than 280 .

Following the specification in section 3.4, most illnesses are minor. The average number of severe illnesses is 5.3 per year; the average number of deaths is .05 per year.

\subsubsection{Among sites, among meal and recipe types and among growth conditions}

Distributions for the number of illnesses among sites (Table 13), among egg meal and egg recipe types (Table 14, Table 15) and among $S$. Enteritidis growth scenarios (Table 16, Table 17) indicate the breakdowns that have a larger share of the number of illnesses than share of 


\begin{tabular}{|c|c|c|}
\hline Site & \% Illnesses & \% Exposure \\
\hline FSI settings & $42[13,39,78]$ & $24[22,24,27]$ \\
\hline Home settings & $58[22,61,87]$ & $76[73,76,78]$ \\
\hline
\end{tabular}

Table 13. Summary statistics (mean $[5 \%, 50 \%, 95 \%]$ ) for site distributions of fractions of illnesses and exposures.

exposure to contaminated eggs. These breakdowns are due to (combinations of) differences in cooking reductions of $S$. Enteritidis in contaminated eggs prior to consumption (Table 13, Table 15), among-individual exposure to $S$. Enteritidis in a contaminated egg (Table 13, Table 15), and growth conditions for $S$. Enteritidis (Table 17).

\section{Among sites}

Egg meal and egg recipe consumption at FSI settings make up $24 \%$ of table egg consumption, compared to larger proportions (42\%) of the number of $S$. Enteritidis in egg associated illnesses (Table 13).

For some types of egg meals and egg recipes, model inputs specify small pools $(\leq 3$ implicated servings per egg) for home settings and large pools (25-75 implicated servings per egg) for FSI settings. At FSI settings, several illnesses could accrue from a single contaminated egg when used in a pooled egg recipe or meal that makes a large number of servings and

- under conditions with no $S$. Enteritidis growth: very rarely from a well-cooked, rarely from a lightly-cooked egg meal or recipe, but occasionally from an uncooked recipe;

- under conditions of moderate $S$. Enteritidis growth: rarely from a well-cooked meal or recipe, occasionally from a lightly-cooked egg recipe and commonly from a raw recipe; and,

- under conditions of maximum $S$. Enteritidis growth: occasionally from a well-cooked egg recipe and frequently to almost always from an egg meal or uncooked or lightly-cooked egg recipe.

Table 13's results, then, show also how much the fraction of illnesses attributable to FSI settings varies among, nominally, years. In some $5 \%$ of nominal years, fewer than $13 \%$ of illnesses are attributable to FSI settings; in some $5 \%$ of nominal years, more than $78 \%$ of illnesses are attributable to FSI settings.

\section{Among egg meal and recipe types}

As per Table 14, accounting for preparation practices, pooling practices, cooking practices (illnesses per contaminated egg, consumption) and consumption practices (illnesses):

- egg meals -fried, scrambled, boiled or poachedaccount for higher proportions of $S$. Enteritidis in egg associated illnesses than egg recipes for home consumption, but egg recipes account for higher proportions of $S$. Enteritidis in egg associated illnesses than egg meals for FSI consumption;

- egg meals and egg recipes that involve less cooking and smaller $S$. Enteritidis reduction (boiled eggs, raw and lightly cooked egg recipes), and larger egg pools (FSI consumption egg meals and egg recipes) have larger number of illnesses per contaminated egg; and,

- egg meals and egg recipes that make up smaller proportions of total shell egg consumption (raw egg recipes, scrambled egg meals, susceptible population) lead to smaller number of illnesses, although sometimes disproportionately to the consumption frequency (raw and lightly cooked egg recipes, boiled egg meals at home settings, scrambled egg meals at FSI settings).

Table 15's results show also how much the fraction of illnesses attributable to different egg meals and egg recipes varies among, nominally, years. In some $5 \%$ of nominal years, $0 \%$ of illnesses are attributable to eggs consumed raw, owing to low exposure to egg meals and recipes with raw eggs; in some $5 \%$ of nominal years, more than $6.7 \%$ of illnesses are attributable to eggs consumed raw, despite low exposure to egg meals and egg recipes with raw eggs. In some $5 \%$ of nominal years, fewer than $16 \%$ of illnesses are attributable to boiled eggs; in some $5 \%$ of nominal years, more than $64 \%$ of illnesses are attributable to boiled eggs.

\begin{tabular}{|c|c|c|c|c|}
\hline \multicolumn{2}{|c|}{ Meals \& recipes } & & Pr\{illness | SE-egg\} & Illnesses per $S E$-egg \\
\hline \multirow{6}{*}{$\frac{\frac{n}{\pi}}{\sum^{\pi}}$} & \multirow{2}{*}{ Fried } & Normal & $6.5 \times 10^{-3}\left[1.8 \times 10^{-6}, 1.6 \times 10^{-4}, .013\right]$ & \multirow{2}{*}{$.0064[0, .0056, .0056]$} \\
\hline & & Susceptible & $9.1 \times 10^{-3}\left[3.3 \times 10^{-6}, 2.9 \times 10^{-4}, .022\right]$ & \\
\hline & \multirow{2}{*}{ Scrambled } & Normal & $3.4 \times 10^{-3}\left[2.4 \times 10^{-7}, 1.1 \times 10^{-5}, 3.3 \times 10^{-4}\right]$ & \multirow{2}{*}{$.012[0, .0056, .036]$} \\
\hline & & Susceptible & $4.7 \times 10^{-3}\left[3.8 \times 10^{-7}, 1.8 \times 10^{-5}, 5.7 \times 10^{-4}\right]$ & \\
\hline & \multirow{2}{*}{ Boiled } & Normal & $.021\left[5.1 \times 10^{-9}, 3.4 \times 10^{-3}, .066\right]$ & \multirow{2}{*}{$.021[0, .0056, .038]$} \\
\hline & & Susceptible & $.031\left[8.2 \times 10^{-9}, 3.3 \times 10^{-3}, .11\right]$ & \\
\hline \multirow{6}{*}{ 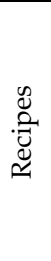 } & \multirow{2}{*}{ Raw } & Normal & $.068[.013, .063, .11]$ & \multirow{2}{*}{$.50[.061, .31,1.4]$} \\
\hline & & Susceptible & $.13[.058, .12, .20]$ & \\
\hline & \multirow{2}{*}{ Lightly cooked } & Normal & $.011\left[4.0 \times 10^{-5}, 1.9 \times 10^{-3}, .028\right]$ & \multirow{2}{*}{$.12[.057, .086, .25]$} \\
\hline & & Susceptible & $.017\left[1.1 \times 10^{-4}, 4.5 \times 10^{-3}, .051\right]$ & \\
\hline & \multirow{2}{*}{ Well cooked } & Normal & $1.4 \times 10^{-4}\left[1.2 \times 10^{-14}, 2.7 \times 10^{-11}, 6.8 \times 10^{-8}\right]$ & \multirow{2}{*}{$.020[0,0, .011]$} \\
\hline & & Susceptible & $3.3 \times 10^{-4}\left[2.7 \times 10^{-14}, 5.6 \times 10^{-11}, 1.3 \times 10^{-7}\right]$ & \\
\hline
\end{tabular}

Table 14. Summary statistics (mean [5\%, 50\%, 95\%]) for egg meal and recipe distributions of the probability of illnesses from consumption of $S$. Enteritidis contaminated egg and illnesses per $S$. Enteritidis contaminated egg. 


\begin{tabular}{|c|c|c|c|}
\hline \multicolumn{2}{|c|}{ Meals \& recipes } & \% Illnesses & \% Exposure \\
\hline \multirow{3}{*}{ Meals } & Fried & $17[5.8,17,31]$ & $31[29,31,34]$ \\
\cline { 2 - 4 } & Scrambled & $9.8[0,6.9,30]$ & $14[12,14,16]$ \\
\cline { 2 - 4 } & Boiled & $39[16,38,64]$ & $20[17,20,24]$ \\
\hline \multirow{3}{*}{ Recipes } & Raw & $1.8[0, .85,6.7]$ & $.048[0, .044, .11]$ \\
\cline { 2 - 4 } & $\begin{array}{c}\text { Lightly } \\
\text { cooked }\end{array}$ & $28[6.7,22,63]$ & $4.4[3.4,4.4,5.4]$ \\
\cline { 2 - 4 } & Well cooked & $3.8[0,2.4,13]$ & $30[28,30,32]$ \\
\hline
\end{tabular}

Table 15. Summary statistics (mean $[5 \%, 50 \%, 95 \%]$ ) for egg meal and recipe distributions of fractions of illnesses and exposures.

Table 15's results show also how the effect of cooking reduces the risk of illness from consuming an $S$. Enteritidis contaminated egg. However, some illnesses accrue, still, from even those egg meals and egg recipes to which one attributes the strongest protection.

\section{Among growth conditions}

Under baseline storage and handling time and temperature specifications, $100 \%$ yolk membrane breakdown seldom occurs, $S$. Enteritidis growth seldom occurs $(4.5 \%)$ and $S$. Enteritidis growth to saturation seldom occurs $(.6 \%)$. However, contaminated eggs stored and handled under conditions that permit $S$. Enteritidis growth to high levels account for disproportionately higher fractions of the number of illnesses (Table 17).

Absolute control over the duration and temperature of storage of eggs to prevent $S$. Enteritidis growth in contaminated eggs would reduce the number of $S$. Enteritidis in egg associated illnesses, but would be limited to that of the baseline levels indicated in the No growth columns of Table 17. This is partly due to eliminating the occurrence of large numbers of illnesses from a single $S$. Enteritidis contaminated egg in a pooled egg meal or recipe.

\subsubsection{Sensitivity}

The influential variables for the number of illnesses, illnesses per contaminated egg and egg prevalence and their order of importance are:

- number of illnesses: egg prevalence, illnesses per $S$. Enteritidis egg;
- illnesses per $S$. Enteritidis egg: servings per egg and Pr\{illness ISE-egg\}; and,

- egg prevalence (section 4.2.2): flock prevalence, sizes of $S$. Enteritidis positive flocks, egg-within flock prevalence.

$S$. Enteritidis egg prevalence and the number of illnesses per $S$. Enteritidis egg have multiplicative effects on the number of illnesses: twice the egg prevalence effects twice the number of illnesses; twice the number of illnesses per contaminated egg effects twice the number of illnesses.

\section{$S$. Enteritidis-egg prevalence}

The number of illnesses is proportional to the egg prevalence such that when egg prevalence decreases by some percentage, the number of illnesses decreases by the same percentage; when egg prevalence increases by some percentage, the number of illnesses increases by the same percentage. Figure 7 illustrates the conditional distribution of the number of illnesses given egg prevalence in the lower $20 \%$ of the egg prevalence distribution, the next $20 \%$ of the egg prevalence distribution, etc.

Larger egg prevalence shifts the distribution for the number of illnesses to higher values, but makes the number of illnesses, nominally in a year, also more variable. Contrast the spread of the distribution for the number of illnesses conditional on the lower one-fifth of the egg prevalence distribution and the spread of the distribution for the number of illnesses conditional on the upper fifth of the egg prevalence distribution.

All of the following characteristics that have a multiplicative effect on the egg prevalence (section 4.2.2)

- doubling flock prevalence doubles egg prevalence; halving flock prevalence halves egg prevalence;

- doubling egg within flock prevalence doubles egg prevalence, halving egg within flock prevalence halves egg prevalence; and,

- doubling the sizes of infected flocks doubles the number of illnesses, halving the size of infected flocks halves egg prevalence

also have a multiplicative effect on the number of illnesses

- doubling flock prevalence doubles the number of illnesses; halving flock prevalence halves the number of illnesses;

\begin{tabular}{|c|c|c|c|}
\hline $\begin{array}{c}\text { Growth } \\
\text { conditions }\end{array}$ & & $\operatorname{Pr}\{$ illness $\mid$ SE-egg $\}$ & Illnesses per $S E$-egg \\
\hline \multirow{2}{*}{ No growth } & Normal & $4.4 \times 10^{-3}\left[2.6 \times 10^{-13}, 1.2 \times 10^{-5}, .033\right]$ & \multirow{2}{*}{$5.4 \times 10^{-3}\left[0,1.9 \times 10^{-4}, .031\right]$} \\
\hline & Susceptible & $7.2 \times 10^{-3}\left[4.2 \times 10^{-13}, 1.2 \times 10^{-5}, .056\right]$ & \\
\hline \multirow{2}{*}{ Some growth } & Normal & $9.4 \times 10^{-3}\left[2.4 \times 10^{-13}, 1.4 \times 10^{-5}, .017\right]$ & \multirow{2}{*}{$.019\left[0,1.9 \times 10^{-4}, .044\right]$} \\
\hline & Susceptible & $.013\left[2.2 \times 10^{-5}, .0023, .040\right]$ & \\
\hline \multirow{2}{*}{$\begin{array}{l}\text { Growth to } \\
\text { saturation }\end{array}$} & Normal & $.51\left[4.4 \times 10^{-5}, .56,1\right]$ & \multirow{2}{*}{$1.4[.86,1.3,2.2]$} \\
\hline & Susceptible & $.56\left[7.8 \times 10^{-5}, .71,1\right]$ & \\
\hline
\end{tabular}

Table 16. Summary statistics (mean $[5 \%, 50 \%, 95 \%]$ ) for growth conditions distributions of probability of illnesses from consumption of S. Enteritidis contaminated egg and illnesses per $S$. Enteritidis contaminated egg. 


\begin{tabular}{|c|c|c|}
\hline $\begin{array}{c}\text { Growth } \\
\text { conditions }\end{array}$ & $\%$ Illnesses & \% Exposure \\
\hline No growth & $49[19,47,86]$ & $95.6[93.5,95.7,97.3]$ \\
\hline Some growth & $5.5[0,3.6,17]$ & $3.8[2.2,3.7,5.8]$ \\
\hline $\begin{array}{c}\text { Growth to } \\
\text { saturation }\end{array}$ & $46[6.7,48,79]$ & $.56[.080, .45,1.4]$ \\
\hline
\end{tabular}

Table 17. Summary statistics (mean [5\%, 50\%, 95\%]) for growth conditions distributions of fractions of illnesses and exposures.

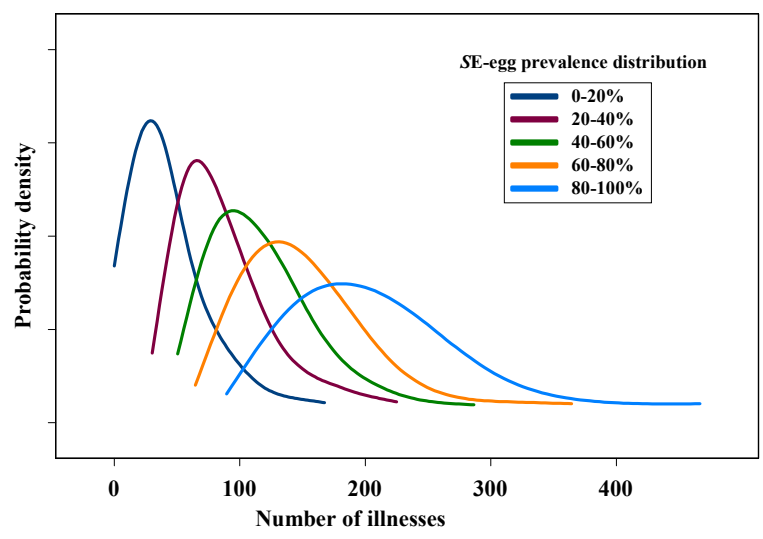

Figure 7. Distribution of the number of illnesses under different egg prevalence specifications.

- doubling egg within flock prevalence doubles the number of illnesses, halving egg within flock prevalence halves the number of illnesses; and,

- doubling the sizes of infected flocks doubles the number of illnesses, halving the size of infected flocks halves the number of illnesses.

\section{Illnesses per contaminated egg}

The number of illnesses is proportional also to the number of illnesses per contaminated egg such that when the number of illnesses per contaminated egg decreases by some percentage, the number of illnesses decreases by the same percentage. Influence of site, meals and recipes and growth conditions on the number of illnesses per contaminated egg is detailed in section 5.1.2.

\section{Site}

At FSI settings, reducing the frequency of egg pooling (that is, reducing the frequency of meals and recipes that pool large numbers of eggs for a large number of servings), particularly for lightly cooked or raw meals and recipes, reduces the number of illnesses per contaminated egg.

At home settings, reducing the frequency of lightly cooked or raw meals and recipes also reduces the number of illnesses per contaminated egg. Egg pools at home settings serve smaller numbers of persons than at FSI settings.
The percentage of illnesses attributable to consumption at FSI settings decreases relative to home settings as

- FSI egg pooling practices change, to reduce the frequency that multiple servings from egg meals and egg recipes are contaminated with $S$. Enteritidis, or to reduce the number of servings that are contaminated by a single contaminated egg.

- different scenarios for the FSI sources (local producer, wholesale, retail) of table eggs lead to shorter storage times, less frequent yolk membrane breakdown, and less $S$. Enteritidis in contaminated shell eggs at consumption.

\section{Meals and recipes}

Two characteristics, particularly, capture the effect of meals and recipes on the illnesses per contaminated egg: cooking $\log _{10}$ reduction and pooling.

Reducing the frequency of egg pooling, that is, reducing the frequency of meals and recipes that pool large numbers of eggs for a large number of servings, particularly for lightly cooked or raw meals and recipes, reduces the number of illnesses per contaminated egg.

Increasing the frequency of meals and recipes that evoke a large $\log _{10}$ reduction of $S$. Enteritidis populations or decreasing the frequency of meals and recipes that involve only small $\log _{10}$ reductions of $S$. Enteritidis populations reduces the number of illnesses per contaminated egg.

\section{Growth conditions}

As per Table 4, growth conditions are represented by three distinguishable parts: contaminated eggs that experience no growth (approximately 95.6\% at baseline conditions); those that experience some growth (approximately 3.8\% at baseline conditions); and those that experience growth to maximum $S$. Enteritidis density (approximately $.6 \%$ at baseline conditions). When conditions that lead to $S$. Enteritidis growth occur less frequently, then the distribution of Prillness I S. Enteritidis egg\} shifts to smaller values and away from the higher values attributed to consumption of eggs with very high $S$. Enteritidis levels. Eliminating all conditions for growth shifts the distribution for Prillness I $S$. Enteritidis egg\} to as small a set of values as possible, that is, those associated with $S$. Enteritidis levels at lay. Further reductions from controlling growth conditions, alone, are not possible.

\section{$S$. Enteritidis egg prevalence versus Illnesses per contaminated egg}

Under conditions which permit even occasional growth of $S$. Enteritidis contamination to saturation levels or to less than saturation levels, the illnesses per contaminated egg is more influential than the egg prevalence for the number of 
illnesses. However, as the fraction of contaminated eggs that experiences some growth decreases, the influence of egg prevalence relative to the number of illnesses per contaminated egg increases. Under conditions where no growth of $S$. Enteritidis occurs from initial contamination levels, illnesses per contaminated egg and egg prevalence exert the same relative influence on the number of illnesses.

\section{Model sensitivity}

Other precedent risk assessments' choices for modeling can be used to provide a measure of the sensitivity of the conclusions from sections 5.1.1-5.1.3 for this implementation.

Alternative S. Enteritidis contamination and growth Alternative specifications for $S$. Enteritidis deposition at lay [5] and growth immediately after lay [8] lead to $17 \%$ higher and $41 \%$ lower mean stable $S$. Enteritidis in egg concentrations after lay and to $13 \%$ more and $7 \%$ fewer illnesses per contaminated egg than in the baseline case used here (data not shown).

Alternative specifications for growth following 100\% yolk membrane breakdown and the maximum $S$. Enteritidis in a contaminated egg lead to very small differences in the number of illnesses per contaminated egg (data not shown).

\section{Alternative pooling characteristics}

Alternative pooling characteristics in home and FSI settings from USDA [8] lead to $19 \%$ lower illnesses per contaminated egg (ratio of distribution means), $2 \%$ lower probability of illness from eating a serving from a contaminated egg (ratio of distribution means), and 19\% lower number of illnesses (ratio of distribution means) than under baseline conditions (data not shown).

\begin{tabular}{|c|c|}
\hline Dose-response model & Illnesses per contaminated egg \\
\hline Weibull model & $\begin{array}{c}1.4 \times 10^{-2}\left[5.1 \times 10^{-3}, 8.2 \times 10^{-3},\right. \\
\left.4.6 \times 10^{-2}\right]\end{array}$ \\
\hline USDA Beta-Poisson, normal [5] & $\begin{array}{c}1.9 \times 10^{-2}\left[2.2 \times 10^{-3}, 9.6 \times 10^{-3},\right. \\
\left.6.1 \times 10^{-2}\right]\end{array}$ \\
\hline $\begin{array}{l}\text { USDA Beta-Poisson, } \\
\text { susceptible [5] }\end{array}$ & $4.2 \times 10^{-2}\left[3.4 \times 10^{-3}, .016,2.0 \times 10^{-1}\right]$ \\
\hline $\begin{array}{l}\text { FAO/WHO Beta-Poisson, Mid } \\
\text { [7] }\end{array}$ & $\begin{array}{c}9.1 \times 10^{-3}\left[1.3 \times 10^{-3}, 6.1 \times 10^{-3},\right. \\
\left.2.3 \times 10^{-2}\right]\end{array}$ \\
\hline $\begin{array}{l}\text { FAO/WHO Beta-Poisson, } \\
\text { Lower [7] }\end{array}$ & $\begin{array}{c}7.0 \times 10^{-3}\left[8.5 \times 10^{-4}, 4.4 \times 10^{-3},\right. \\
\left.1.7 \times 10^{-2}\right]\end{array}$ \\
\hline $\begin{array}{l}\text { FAO/WHO Beta-Poisson, } \\
\text { Upper [7] }\end{array}$ & $\begin{array}{c}1.2 \times 10^{-2}\left[1.7 \times 10^{-3}, 7.7 \times 10^{-3},\right. \\
\left.3.2 \times 10^{-2}\right]\end{array}$ \\
\hline USDA Beta-Poisson, m.l.e. [8] & $\begin{array}{c}9.1 \times 10^{-3}\left[1.3 \times 10^{-3}, 6.0 \times 10^{-3},\right. \\
\left.2.3 \times 10^{-2}\right]\end{array}$ \\
\hline
\end{tabular}

Table 18. Alternative dose-response models' effect on distribution for the number of illnesses per contaminated egg. Entries are summary statistics (mean [5\%,50\%, 95\%] points from distribution (among contaminated eggs) for illnesses per contaminated egg).
Pooling characteristics like the ones in USDA [8], with smaller egg pools for egg meals and recipes at FSI settings, larger egg pools for egg meals and recipes at home settings, and less frequent use of pooled shell eggs, shifts the distribution of the percentage illnesses attributable to FSI settings in Table 13 to smaller values.

\section{Alternative dose-response models}

The dose-response model choice affects risk outputs for illnesses per contaminated egg (Table 18). As discussed in section 3.3, relative to the USDA Beta-Poisson dose-response model [8], for example, the Weibull dose-response model attributes higher probability of illness at low doses and at high doses for the normal population individuals and at all $S$. Enteritidis doses for the susceptible population individuals (section 6.2).

\subsection{Data gaps and research needs}

The following list of data gaps applies to this and other $S$. Enteritidis risk assessments concerning shell eggs [1], [4], [13]:

- among-flock and egg within positive flock $S$. Enteritidis prevalence;

- in-practice design of surveillance and monitoring, test sensitivities and specificities,

- S. Enteritidis prevalence in breeder and pullet flocks, their environments and their feed;

- location of egg contamination in egg contents;

- rate of breakdown of the yolk vitelline membrane and rate of growth of $S$. Enteritidis at nominal storage temperatures and under conditions of abuse;

- S. Enteritidis growth kinetics, including lag times and growth rates, as they change with different physiological states of the pathogen;

- egg storage and handling times and temperatures;

- cooking practices, meal and recipe allocation, consumption practices and behaviour in food handling and preparation among individuals and among settings;

- cooking reduction, cooking time, method and temperature effects on $S$. Enteritidis; and,

- dose-response, including virulence mechanisms and infectious doses, epidemiological data for the number of illnesses due to $S$. Enteritidis in shell eggs, and variability in human response to exposure to S. Enteritidis.

The scope of this risk assessment-excluding some sources of shell eggs, egg products, and externally contaminated eggs-excludes many of the data gaps and research needs that precedent risk assessments reported for other grades of shell eggs, other sources of shell eggs and other contamination pathways. 


\subsection{Points for discussion}

\subsubsection{Scope}

This risk assessment describes the risk of illness from consumption of Canadian grade A table eggs internally contaminated with $S$. Enteritidis, where the eggs are from regulated flocks under the supply management system operating in Canada.

Grade A table eggs offered for sale in Canada can come from several other sources: shell egg imports from the United States, hatchery surplus eggs from layer- and broiler-breeder flocks, and shell eggs from smaller flocks that are not regulated under the supply management system. Other grades of eggs can also come from all those sources. Although eggs produced outside of the supply management system are an important consideration in the burden of illness of $S$. Enteritidis in table eggs, they could not be included in this assessment due to a lack of information on flock prevalence and flock management practices.

Also outside the scope are $S$. Enteritidis in egg-associated illnesses from the following:

- externally (shell) contaminated eggs and subsequent shell penetration by $S$. Enteritidis;

- other grades of eggs, dirty and cracked eggs;

- farm-gate sales and farmers' markets sales;

- processed egg products; and,

- cross-contamination in food cooking and preparation practices resulting in illness.

\subsubsection{Change in flock prevalence}

The CEMA data [39], [54] suggest smaller S. Enteritidis flock prevalence at present than in the early 1990's, subject to the following cautions

- Poppe et al. [48]: described a single point in time survey of flock infection, with environment testing for $S$. Enteritidis (and other Salmonella spp.); the published result, however, does not permit inferences beyond the 295 flocks that those authors tested, in its presented form (no design-unbiased estimator), despite having a well-defined reference (commercial laying flocks), sampling population (list of 1682 producing commercial egg-laying operations) and sample design, except by extrapolation; no measure of uncertainty, even for the single point in time, can be constructed.

- CEMA flock prevalence data [39], [54] used here represent two single year data sets of flocks with environmental tests for $S$. Enteritidis; results, however, do not permit inference beyond the flocks tested to reference population (nominally, commercial laying flocks), in their presented form, due lack of design information (self-selection), appropriate estimator and unknown test sensitivity.
Changes resulting from industry activity (section 2.3) and increased industry awareness could be the reason for the observed reduction in flock prevalence.

\subsubsection{Baseline conditions as appropriate}

for examining risk management options

Section 1 introduced some of the issues encountered with using expert opinion, anecdotal information, and information extrapolated from other countries' practices to characterize the risk of illnesses from $S$. Enteritidis in shell eggs. The risk characterization makes up for some of that lack of information by describing how the risk changes within what are set as baseline conditions, and how the risk changes under other descriptions of current practice that are deemed to hold in other countries but might or might not hold in Canada.

\subsubsection{Lack of information to describe uncertainty}

Notably lacking is any formal statement of the uncertainty that we would associate with the risk outputs produced in sections 5.1.1-5.1.3. Most data used to inform a model of the risk come with no formal statement of uncertainty; formal statements of data uncertainty cannot be made for many of the other data used to inform a model of the risk.

\subsubsection{Implication of results' variability for policy initiatives}

Results for baseline conditions (section 5.1.1,5.1.2) and the examination of sensitivity of the risk outputs to various influential variables (section 5.1.3) point to possibilities for risk management options: egg prevalence; cooking reduction; pooling practices; and, storage and handling.

Factors that keep the probability of illness small from eating a serving, at random, from a contaminated egg, at random, are:

- growth controlled so that $100 \%$ yolk membrane breakdown does not occur and growth from initial deposition at lay does not occur;

- small frequency of occurrence of pooling eggs for egg meals and egg recipes or only small numbers of eggs are used in shared egg meals and egg recipes; and,

- small frequency of lightly cooked or raw egg dishes.

Factors that keep the probability of illness small from eating a serving, at random, from an egg, at random, are the same as above plus:

- low egg prevalence, through low flock prevalence, low egg within flock prevalence and control of the total number of hens laying in positive flocks. 


\subsubsection{Number of illnesses}

The modeled annual number of $S$. Enteritidis egg associated illnesses (section 5.1.1) under baseline conditions is approximately $1 \%$ of the epidemiologicalbased estimate for the number of $S$. Enteritidis-associated illnesses per year from section 2.2.1, at the 2 results' mean values. Several factors put the comparison into context:

- the modeled number of illnesses respects the scope (0), which limits both the source of the eggs -grade A eggs from regulated flocks under the supply management system-and the nature of the contamination -internally contaminated with S. Enteritidis;

- the modeled number of illnesses represents that number under a set of baseline conditions; those baseline conditions are deemed to resemble current practices, except where noted, but cannot be demonstrated to do so; and,

- data limitations let us attach no measure of uncertainty to the modeled number of illnesses of $S$. Enteritidis egg associated illnesses.

\subsubsection{Comparisons to other risk assessments} for S. Enteritidis in eggs

The prevalence distribution of eggs from the regulated flocks internally contaminated with $S$. Enteritidis has a mean of 1.7 per million table eggs, $5 \%$ point 4 per 10 million eggs and $95 \%$ point 3.1 per million eggs (section 4.2.1). Comparisons to results from other risk assessments are added for context. Results reflect different flock management practices in different countries, different detection practices in different countries or other differences as noted.

- USDA [8] (by simulation) reported approximately 278 internally or externally contaminated eggs per 1 million shell eggs of any type, from any source for the United States.

- Kelly et al. [12] (by simulation) reported approximately 89 internally or externally contaminated eggs per 1 million shell eggs produced on the island of Ireland, [21, 240] per 1 million shell eggs (95\% Bayesian posterior interval).

- ESFA [14] reported flock (environment) positive prevalence for $S$. Enteritidis that varied from 0 (0 of 676 tested, 0 of 626 tested, 0 of 590 tested, 0 of 40 tested, 0 of 10 tested, 0 of 1 tested) to $25 \%$ ( 2 of 8 tested), varying among EU member states for the 2007 reporting year, with median value $3.5 \%$, among 21 member states.

\section{Examination of Risk Management Options}

The number of illnesses is primarily determined by the egg prevalence and the number of illnesses per contaminated egg. These in turn form the basis for the examination of risk management options, the effect that two flock management practices have on the prevalence of contaminated eggs (section 6.1) and the effect that egg storage, handling, and preparation practices have on the number of illnesses per contaminated egg (section 6.2).

Risk management option risk outputs are subject to the same sources of variability and uncertainty as the risk outputs calculated under a designated set of baseline conditions that the risk management options are compared to, blocked within the same baseline condition. Calculated effects are a distribution of reductions effected by the risk management over the distribution of baseline conditions.

\subsection{Flock management practices that lead to decreased egg prevalence}

From section 5.1.3, the number of illnesses is proportional to the egg prevalence (flock prevalence or within-flock prevalence) such that when the prevalence of $S$. Enteritidis positive table eggs decreases, the number of illnesses decreases by the same percentage. Risk management options are aimed at reducing $S$. Enteritidis prevalence.

\subsubsection{Vaccination programs}

\section{Introduction}

In Canada, laying flocks are not routinely vaccinated. In 2006, for example, approximately $4 \%$ of the hens in regulated laying flocks were in vaccinated flocks [39]. In some jurisdictions, there may be targeted vaccination to protect a flock deemed to be at higher risk of infection, say, if the previously housed flock was found positive for S. Enteritidis, S. Enteritidis was not completely eliminated from the environment by cleaning and disinfection procedures, or the organism might be reintroduced by a vector or other source.

\section{Methods}

Vaccination strategies are simplified to:

- the fraction of flocks in the flock population that are vaccinated; and,

- how the flocks to be vaccinated are selected: at random, by size (the largest flocks), and flocks that have higher probability of becoming infected. 
We evaluate vaccination strategies' effectiveness by comparing population overall egg prevalence under the vaccination strategy to the population overall egg prevalence under the baseline conditions, which include no vaccination (section 4.2.1, 5.1.1). Section 5.1.3 linked egg prevalence and the number of illnesses.

To simplify the comparison, we simplify the inference for the vaccination effect to

- within-flock egg prevalence for a vaccinated flock is $4 \%$ of that for a non vaccinated flock (section 2.5.2; Figure 8); and,

- there is no variability in the vaccination effectiveness between flocks, in timing of vaccination in relation to age at which possible exposure occurs, in vaccination protocols, or in types of vaccines

so that the egg within flock prevalence distribution under a strategy that vaccinates some flocks becomes a mixture of two egg-within-flock prevalence distributions (data not shown).

\section{Results}

If vaccination strategies have no effect, the egg prevalence under the vaccination strategy will be exactly $100 \%$ of the egg prevalence under baseline conditions. This will always be the case when no flocks are vaccinated and will never be the case when all flocks are vaccinated. In cases where only some of the flocks are vaccinated, a vaccination strategy would have no effect:

- nearly always: when few flocks are vaccinated and among flock prevalence is very low;

- less often: when more flocks are vaccinated, even when vaccination is not targeted or targeting is ineffective; and,

- almost never: when most flocks are vaccinated.

If vaccination strategies have at least some effect, egg prevalence will be $<100 \%$ that of baseline conditions. This will always be the case when all flocks are vaccinated and will never be the case when no flocks are vaccinated. In cases where only some of the flocks are vaccinated, a vaccination strategy would have at least some effect:

- almost never: when few flocks are vaccinated or when among flock prevalence is very low;

- more often: when more flocks are vaccinated, even when vaccination is not targeted or when targeting is ineffective; and,

- almost always: when most flocks are vaccinated.

Density in the egg prevalence distribution shifts from higher egg prevalence to lower egg prevalence values, particularly, when some flocks are vaccinated, reducing the frequency of high egg prevalence (illustrated in Figure 9 for Case 1 in Table 19).

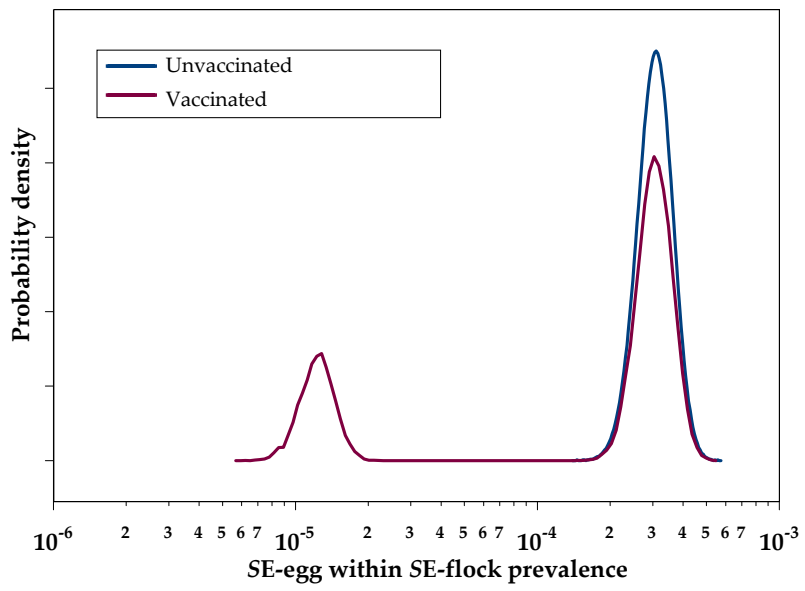

Figure 8. Distribution of contaminated egg within flock prevalence with or without flock vaccination.

The effect is stronger when a higher percentage of infected flocks are vaccinated, when a higher percentage of larger flocks are vaccinated and when a higher percentage of infected are vaccinated. Illustrating at the distributions' mean egg prevalence, the decrease in egg prevalence varies, from as little as no decrease (the same as baseline egg prevalence), to as much as $4 \%$ of baseline egg prevalence (Table 19). Average egg prevalence under the vaccination strategies varies from $4 \%$ to $91 \%$, as a fraction of average egg prevalence under baseline conditions (no vaccination). The average egg prevalence decreases as the fraction of vaccinated flocks in the flock population increases (among Table 19 columns, left to right along any row), and decreases as the application of the vaccination strategy includes higher percentages of larger flocks (Table 19, Case 2 versus Case 1, any column), except when $0 \%$ or $100 \%$ of flocks are vaccinated.

Vaccination strategies that target larger flocks reduce within-flock egg prevalence for more of the table egg production; more of the table egg production is targeted when vaccination is applied to the largest $10 \%, 50 \%$ or $90 \%$ of flocks (Table 19, Case 2) than when flocks are vaccinated or not vaccinated without regards to flock size (Table 19, Case 1). However, vaccination strategies that are directed towards larger flocks but that are not applied to all flocks still can result in no egg prevalence reduction when none of the flocks that become infected happen to also be among the vaccinated flocks. 


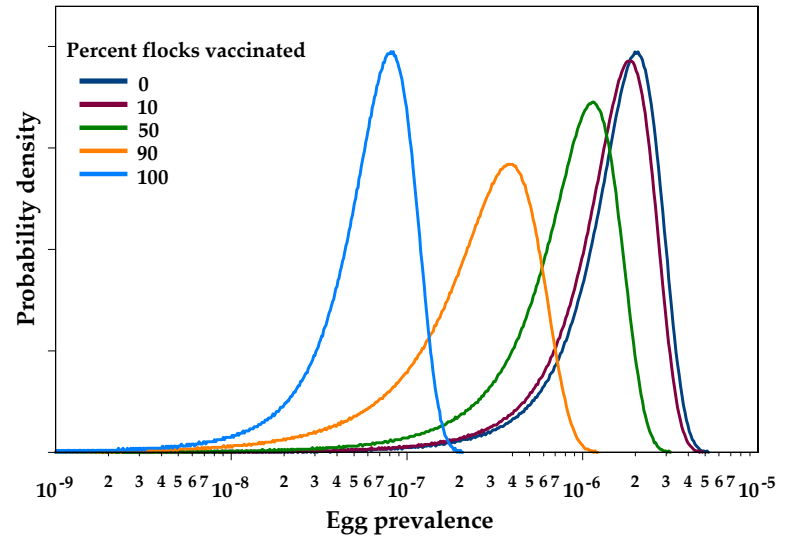

Figure 9. Distribution of mean egg prevalence under flock vaccination.

Table 19, Case 3 mimics targeted vaccination strategies that may be used to afford protection to an incoming layer flock deemed to be at higher risk of infection. Strategies' effectiveness range from ones completely effective at identifying flocks that would become infected (Table 19, Case 3, 100\% infected flocks vaccinated) to ones that are completely ineffective at identifying flocks that would become infected (Table 19, Case 3, 0\% infected flocks vaccinated).

A theoretical strategy that could perfectly target vaccination at the infected flocks - e.g., a flock moving into a house that was occupied by a previously positive flockcan achieve the same levels of average egg prevalence reduction as $100 \%$ flock vaccination strategies, but the targeted strategies' effectiveness decreases as targeted vaccination strategies miss some infected flocks. Targeted vaccination effects approximately the same reduction as does vaccinating the same percentage of the whole layer flock population (Table 19, Case 3 versus Case 1).

\section{Sensitivity}

Results in Table 19 rely on assumptions underlying the baseline conditions. Particularly, if flock prevalence does increase with flock size, then egg prevalence is higher for the same flock prevalence in both baseline conditions and in the simulated results of applying the vaccination strategy. Relative change in egg prevalence between any particular vaccination strategy and baseline results remains the same as shown.

If flock vaccination effectiveness varies, and withinvaccinated flock egg prevalence is $4 \%$ of within nonvaccinated flock egg prevalence, then average effects remain nominally as in Table 19, but the distribution of percentage effectiveness outputs becomes more variable.

Reduction variability increases, even while the average egg prevalence reduction remains the same, as flock prevalence decreases and as variability in egg within

\begin{tabular}{|c|c|c|c|c|c|c|}
\hline \multirow{2}{*}{ Case } & \multirow{2}{*}{ Application } & \multicolumn{5}{|c|}{ \% flocks vaccinated } \\
\cline { 3 - 7 } & & 0 & 10 & 50 & 90 & 100 \\
\hline 1 & $\begin{array}{c}\text { Without regard to } \\
\text { size, infection status }\end{array}$ & 100 & 90 & 55 & 17 & 4 \\
\hline 2 & Largest flocks & 100 & 86 & 30 & 13 & 4 \\
\hline 3 & Infected flocks & 100 & 91 & 55 & 18 & 4 \\
\hline
\end{tabular}

Table 19. Mean egg prevalence (\% of baseline, no vaccination) under vaccination strategies.

flock prevalence increases. Egg prevalence reductions reported in Table 19 are smaller, as a percentage of baseline, if vaccination applied is less effective in reducing within-flock prevalence; egg prevalence reductions reported in Table 19 are larger, as a percentage of baseline, if vaccination is more effective in reducing within-flock prevalence than assumed.

Vaccination strategies' effectiveness, even with strategies that do not target flocks with deemed higher risk of infection, improve towards $4 \%$ faster with increases in the percentage of vaccinated flocks, as flock prevalence increases and improves towards $4 \%$ slower with increases in the percentage of vaccinated flocks, as flock prevalence decreases.

The available literature permits an inference about the average ratio of contaminated egg within non-vaccinated flock to contaminated egg within vaccinated flock prevalence of 25 , that has $95 \%$ confidence interval [2.58, 254.7], approximately $1 / 10$ at the lower limit and approximately $10 \times$ higher at the upper limit of the nominal ratio (data not shown).

\section{Discussion points}

Targeted vaccination is differentiated from routine vaccination, which Table 19's Cases 1 and 2 mimic. Farms using an all-in all-out approach are more likely able to control $S$. Enteritidis using cleaning and disinfection, but incoming flocks would still be susceptible to sources of $S$. Enteritidis other than a contaminated environment from the previously housed positive flock. If the source of infection could not be identified, it is possible that the same source could result in reintroduction of infection to the subsequent layer flock. Therefore, targeted vaccination may provide an additional degree of protection to the subsequent flock.

Information about current flock vaccination practices is limited (section 2.3). However, current practices are included within the range of alternatives considered in this section.

\subsubsection{Flock testing and egg diversion from positive flocks}

\section{Introduction}

Some jurisdictions mandate flock testing for $S$. Enteritidis and divert eggs from a detected positive flock away from the table egg market. 


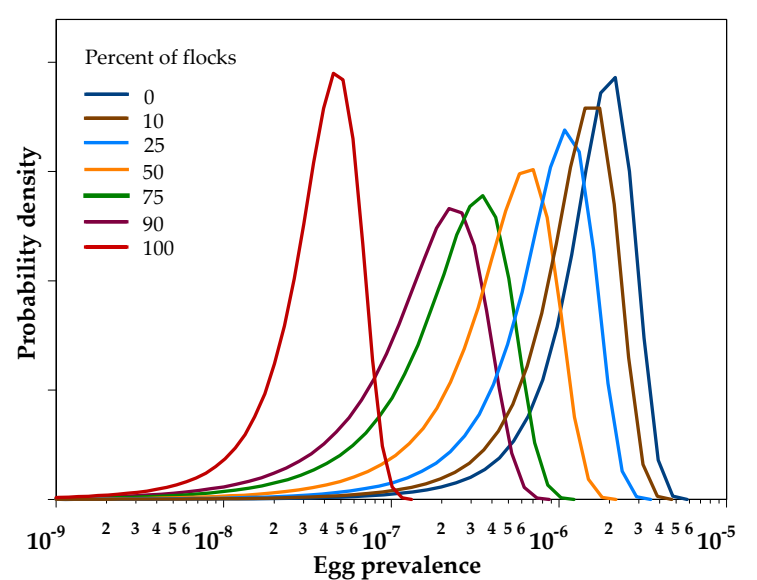

Figure 10. Egg prevalence distribution under a test and divert flock management strategy, with an environmental test at the beginning and one 8 to 10 weeks before the end of the laying cycle. Infection onset is prior to the beginning of the laying cycle.

\section{Methods}

We evaluate the effect that flock environment testing and diverting eggs from an environmentally positive flock has on the number of illnesses by comparing the egg prevalence under several test and divert regimens to the egg prevalence under baseline conditions, in which no egg diversion is included. Section 5.1.3 linked egg prevalence and the number of illnesses.

We used the following four test regimens with different levels of practice among flocks:

1. test before beginning of lay cycle so that all production from a flock in an $S$. Enteritidis positive environment diverted;

2. test before the end of lay cycle so that $8-10 \mathrm{wk}$ of production from a flock in an $S$. Enteritidis positive environment diverted;

3. both Regimen 1 and Regimen 2;

4. Regimen 3 and an additional test between the two.

\section{Results}

Density in the egg prevalence distribution, varying among nominal years, shifts from higher egg prevalence to lower egg prevalence values and reduces the frequency of high population overall egg prevalences, particularly, when some flocks practise a test and divert management (illustrated in Figure 10 for Case 3 in Table 20).

The effect is stronger when a higher percentage of flocks practise test and divert and when a higher percentage of larger flocks practise test and divert.

Illustrating at the distributions' mean egg prevalence, the mean egg prevalence

- varies among test regimens (Table 20, rows); it decreases as a percentage of the baseline case's egg prevalence, generally, as the number of tests within

\begin{tabular}{|c|c|c|c|c|c|c|c|}
\hline \multirow{2}{*}{ Case } & \multirow{2}{*}{ Test regimen } & \multicolumn{6}{|c|}{ Percentage of flocks practising } \\
\cline { 3 - 8 } & & 10 & 25 & 50 & 75 & 90 & 100 \\
\hline 1 & Beginning & 91 & 78 & 54 & 31 & 16 & 3 \\
\hline 2 & $8-10 w k$ before end & 98 & 96 & 92 & 88 & 85 & 84 \\
\hline 3 & $\begin{array}{c}\text { Beginning \& } \\
8-10 w k \text { before end }\end{array}$ & 91 & 78 & 54 & 31 & 16 & 2 \\
\hline 4 & $\begin{array}{c}\text { Beginning, 1 } \\
\text { between, 8-10 wk } \\
\text { before end }\end{array}$ & 91 & 77 & 54 & 31 & 16 & 2 \\
\hline
\end{tabular}

Table 20. Egg prevalence as percentage of baseline for 4 test regimens and levels of practice, when flock infection occurs before beginning of lay. Calculated at egg prevalence distribution means.

the layer cycle increases and as the tests happen earlier in egg production; and,

- decreases as the fraction of flocks practising the regimen increases (Table 20, columns),

contingent on assumptions that

- flock infection happens early in flock life, before the beginning egg production;

- the decision to apply the regimen does not depend on flock size; and

- environment testing detects an S. Enteritidis positive environment $90 \%$ of the time (mean).

Environment testing for flocks and diversion of egg production from flocks in an S. Enteritidis positive environment reduces population average egg prevalence from baseline, with no test and divert strategy:

- testing every flock prior to the beginning of lay and diverting eggs from $S$. Enteritidis positive flocks reduces population average egg prevalence to $3 \%$ of baseline levels (Table 20, Case 1);

- adding another test 8-10 wk before the end of lay reduces average egg prevalence to $2 \%$ of baseline levels (Table 22, Case 3); however, testing only at $8-10 \mathrm{wk}$ before end of lay diverts fewer eggs from an $S$. Enteritidis positive flock than testing at the beginning of lay and reduces the average egg prevalence to $84 \%$ of baseline levels (Table 20, Case 2);

- increased frequency of environmental testing during the layer cycle results in larger cumulative reductions, but smaller incremental reductions; and,

- even when participation in testing and diversion is $100 \%$, contaminated eggs remain due to false negative environment tests and tests that are scheduled only some time after the infected flock begins to lay contaminated eggs.

A test and divert practice effects larger reductions in the egg prevalence average when the practice happens at a higher rate among larger flocks (Table 21). The number of contaminated eggs originating from larger flocks would be higher, when within-flock egg prevalence does not vary 


\begin{tabular}{|c|c|c|c|c|}
\hline \multirow{2}{*}{ Case } & Test regimen & \multicolumn{3}{|c|}{$\begin{array}{c}\text { Percentage of flocks practising } \\
\text { regimen (\%) }\end{array}$} \\
\cline { 3 - 5 } & & 25 & 50 & 90 \\
\hline 1 & Beginning & 67 & 46 & 14 \\
\hline 2 & $8-10 w k$ before end & 94 & 90 & 85 \\
\hline 3 & $\begin{array}{c}\text { Beginning \& 8-10wk } \\
\text { before end }\end{array}$ & 67 & 45 & 13 \\
\hline
\end{tabular}

Table 21. Egg prevalence as percentage of baseline when levels of practice increases with flock size. Calculated at egg prevalence distribution means.

with flock size, and the effect of diverting a greater number of eggs from the table market is also greater. The effect disappears as the practice of testing increases to cover $100 \%$ of flocks since all eggs laid in an S. Enteritidis-positive environment would be diverted from the table market, regardless of the flock size. If flock prevalence increases with flock size or if egg within flock prevalence increases with flock size, then the effect of targeting larger flocks is stronger. If flock prevalence decreases with flock size or if egg within flock prevalence decreases with flock size, then the effect of targeting larger flocks is weaker.

Reductions in average egg prevalence are larger when environment test sensitivity is higher (Table 22). The effectiveness lost by using environment testing with low sensitivity for $S$. Enteritidis is offset when the test regimen includes testing on several occasions.

In Canada, sources of flock infection that occur prior to the onset of the laying cycle -exposure to $S$. Enteritidis infection as chicks or pullets- are considered to be less important than sources of infection that occur during the lay cycle (section 1 ). If so, then Table 23 (versus Table 20) demonstrates that reductions from baseline conditions using the test and divert strategy become smaller (illustrated in Figure 11 for Case 3 in Table 23). Reductions from baseline also depend upon the ability to co-ordinate an environmental test with the onset of flock infection (Table 23 versus Table 20).

\begin{tabular}{|c|c|c|c|c|c|c|c|}
\hline \multirow{3}{*}{ Case } & \multirow{3}{*}{ Test regimen } & \multicolumn{3}{|c|}{$\begin{array}{c}\text { Test sensitivity } \\
70 \%\end{array}$} & \multicolumn{3}{|c|}{$\begin{array}{c}\text { Test sensitivity } \\
90 \%\end{array}$} \\
\cline { 3 - 8 } & & \multicolumn{2}{|c|}{$\begin{array}{c}\text { \% flocks } \\
\text { practising } \\
\text { regimen }\end{array}$} & \multicolumn{3}{c|}{$\begin{array}{c}\text { \% flocks } \\
\text { practising } \\
\text { regimen }\end{array}$} \\
\cline { 3 - 8 } & & 25 & 50 & 90 & 25 & 50 & 90 \\
\hline 1 & Beginning & 81 & 63 & 30 & 78 & 54 & 16 \\
\hline 2 & $8-10 w k$ before end & 97 & 93 & 88 & 96 & 92 & 85 \\
\hline 3 & $\begin{array}{c}\text { Beginning \& } \\
\text { 8-10wk before end }\end{array}$ & 81 & 61 & 28 & 78 & 54 & 16 \\
\hline
\end{tabular}

Table 22. Egg prevalence as percentage of baseline (no testing) for different flock environment test sensitivity. Calculated at egg prevalence distribution means.

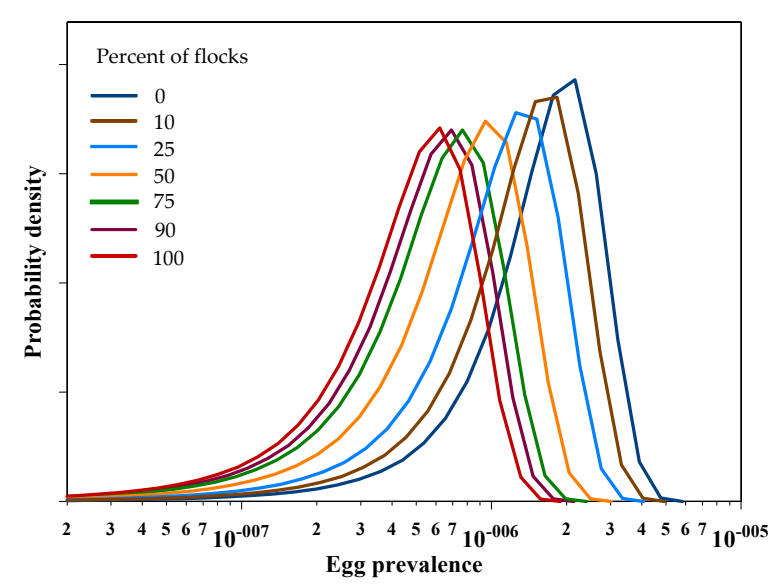

Figure 11. Egg prevalence distribution under a test and divert flock management strategy, with an environmental test at the beginning and one 8 to 10 weeks before the end of the laying cycle. Infection onset is at any age.

\section{Sensitivity}

Results in Table 20-Table 22 and in Table 23 in this section rely on assumptions underlying the baseline conditions, as well. Particularly,

- if flock prevalence does increase with flock size, then egg prevalence is higher for the same flock prevalence in both baseline conditions and in the simulated results of applying a test and divert strategy. Relative change in egg prevalence between any particular test and divert strategy and baseline results remains the same as shown in this section.

- if environment test sensitivity increases as flock size increases [104], then reductions in egg prevalence approach the results in Table 21, which shows larger reductions in prevalence for the same percentage practice than Table 20.

\begin{tabular}{|c|c|c|c|c|c|c|c|c|}
\hline Case & \multirow{6}{*}{ Test regimen } & \multicolumn{6}{|c|}{ regimen (\%) } \\
\cline { 3 - 9 } & & 0 & 10 & 25 & 50 & 75 & 90 & 100 \\
\hline 1 & Beginning & 100 & 94 & 85 & 71 & 55 & 46 & 40 \\
\hline 2 & $\begin{array}{c}8-10 w k \text { before } \\
\text { end }\end{array}$ & 100 & 95 & 88 & 75 & 62 & 55 & 49 \\
\hline 3 & $\begin{array}{c}\text { Beginning \& } \\
8-10 w k \text { before } \\
\text { end }\end{array}$ & 100 & 93 & 83 & 66 & 48 & 37 & 29 \\
\hline 4 & $\begin{array}{c}\text { Beginning, 1 } \\
\text { between, } \\
8-10 w k \text { before } \\
\text { end }\end{array}$ & 100 & 92 & 79 & 59 & 38 & 25 & 15 \\
\hline
\end{tabular}

Table 23. Egg prevalence as percentage of baseline (no testing) when flock infection occurs at any time from age 1 week (pullet stage) to 72 weeks (end of lay). Calculated at egg prevalence distribution means. 


\section{Discussion points}

Results do not account for variability in the rate of production of $S$. Enteritidis-eggs during the laying cycle that one might attribute to the rate of infection among the laying hens in a positive laying environment. Rather, application simplifies those dynamics to ones that distinguish only a point during the flock life cycle before which the environment is not positive and after which the environment is positive.

Egg prevalence under different levels of practice of test and divert strategies is nearly identical to egg prevalence under different levels of practice of vaccination strategies (section 6.1.1). Based on the analysis here, reducing the $S$. Enteritidis contaminated eggs from some fraction of $S$. Enteritidis contaminated flocks from the table egg market by any method appears to evoke the same effect on population egg prevalence among table eggs consumed.

Current flock testing and egg diversion practices vary among provincial jurisdictions (section 2.3, Table 1). However, current practices are included within the range of alternatives considered in this section.

\subsubsection{Sensitivity}

Sections 6.1.1-6.1.2's results for egg prevalence under risk management options as a fraction of baseline egg prevalence hold for any alternative specification of among-flock prevalence and within-flock prevalence. However, outputs in section 6.1 .1 and section 6.1.2, which reflect baseline among-flock prevalence, would change location -move to higher or to lower egg prevalence-or change shape -become more variable or less variable, become more or less skewed-under changes to baseline flock prevalence.

\subsection{Egg preparation and handling practices that reduce the number of illnesses per contaminated egg}

From section 5.1.3, the number of illnesses is proportional to the number of illnesses per contaminated egg such that when the number of illnesses per contaminated egg decreases by some percentage the number of illnesses decreases by the same percentage.

Risk management options are aimed at reducing levels of $S$. Enteritidis in contaminated eggs and at reducing the number of servings from a contaminated egg, particularly, through

- changes to egg storage and handling conditions to reduce growth opportunities and to shift the distribution of growth from higher to lower values (section 6.2.1);

- changes to egg preparation practices to reduce egg pooling (section 6.2.2); and,
- changes to the distribution of egg preparation practices to decrease the fraction of raw and lightly cooked egg meals and recipes among all meals and recipes (section 6.2.3).

\subsubsection{Storage and handling conditions}

\section{Introduction}

Section 5.1.2 Table 17 reported that nearly half of $S$. Enteritidis illnesses are associated with contaminated eggs with maximum levels of $S$. Enteritidis -eggs handled under abusive conditions- despite representing only .6\% of exposures, so that egg storage times and temperatures become important to risk management strategies. In general, managing egg storage and handling for shorter times at colder temperatures reduces the frequency of yolk membrane breakdown and the frequency at which $S$. Enteritidis grows to high levels in a contaminated egg.

\section{Methods}

We evaluate the effect that changes have on the number of illnesses by comparing the number of illnesses per contaminated egg under various storage handling strategies to that of baseline conditions. Section 5.1.3 linked the number of illnesses per contaminated egg and the number of illnesses and identified the influence of

- room temperature storage at home settings;

- growth to intermediate and high levels; and,

- storage time at FSI settings.

Shorter storage times for FSI settings are constructed using the storage time distributions at wholesale and retail, but with the fractions of purchase at local producer, wholesale and retail that Lee et al. [105] reported for a group of restaurants (data not shown).

\section{Results}

Density in the distribution of the number of illnesses per contaminated egg shifts from higher to lower values of number of illnesses per contaminated egg, particularly, when the distribution of the 3 influential factors includes smaller frequency of large values, reducing the frequency of high numbers of illnesses from a single contaminated egg.

Illustrating at the distributions' mean number of illnesses per contaminated egg, several alternatives different from baseline conditions lead to reductions in the number of illnesses per contaminated egg (Table 24):

- at FSI settings, storage for shorter periods of time than baseline conditions leads less frequently to conditions that promote $S$. Enteritidis growth and reduces the number of illnesses per contaminated egg to $70 \%$ of baseline;

- eliminating egg storage at room temperature from baseline $(1.1 \%)$ to $0 \%$ reduces the number of illnesses per contaminated egg to $83 \%$ of baseline at home settings and $67 \%$ of baseline at FSI settings; and 


\begin{tabular}{|c|c|c|}
\hline \multicolumn{2}{|c|}{ Conditions } & $\begin{array}{c}\text { \% Baseline, } \\
\text { at Mean }\end{array}$ \\
\hline \multirow{2}{*}{$\begin{array}{c}\text { Home } \\
\text { settings }\end{array}$} & Baseline time \& temperature & 100 \\
\cline { 2 - 3 } & No room temp. storage & 83 \\
\cline { 2 - 3 } & No growth & 56 \\
\hline \multirow{3}{*}{$\begin{array}{c}\text { FSI } \\
\text { settings }\end{array}$} & Baseline time \& temperature & 100 \\
\cline { 2 - 3 } & Shorter storage time & 70 \\
\cline { 2 - 3 } & No room temp. storage & 67 \\
\cline { 2 - 3 } & No growth & 24 \\
\hline
\end{tabular}

Table 24. Illnesses per contaminated egg as a percentage of baseline conditions under changes to consumer practices.

- eliminating all conditions that lead to yolk membrane breakdown or $S$. Enteritidis growth reduces the number of illnesses per contaminated egg to $56 \%$ of baseline at home settings and to $24 \%$ of baseline at FSI settings.

The largest possible reduction to the number of illnesses per contaminated egg, effected by changes to baseline storage and handling conditions, is represented by the No growth case modeled.

\section{Discussion points}

The data set that Lee et al. [105] reported comes with several caveats:

- the sample was described as a "convenience sample", with no inference about the population to which it refers; convenience samples cannot claim the protection that randomization provides against bias;

- the possible reference population is restaurants that prepare breakfast-type entrées during all hours of operation, located in 13 metropolitan areas in seven states in the United States of America;

- none of the information collected through selfreporting was validated by direct observation;

- applicability of even the demographic information about the establishments is retarded by lack of information about the number of eggs or meals to which the information applies.

So, while we do not consider it an appropriate alternative specification for FSI storage and handling practices -no clear reference population, no clear sampling population, no inference from data to sampling population - we do use it as an example for shorter storage time than baseline. They do demonstrate the effect of shorter storage times on the primary risk outputs, by reducing the frequency of growth of $S$. Enteritidis to high levels in a contaminated egg.

Risk management directed at storage and handling conditions does not affect egg prevalence. So, the strongest effect possible is limited by the prevalence of contaminated eggs handled and stored in ways that limit levels in eggs, at preparation, to the levels at lay.

\subsubsection{Egg pooling}

\section{Introduction}

Reducing the frequency of meals and recipes that pool eggs to make large numbers of servings reduces exposure to $S$. Enteritidis and the number of illnesses per contaminated egg.

\section{Methods}

We evaluate strategies' effectiveness by comparing the number of illnesses per contaminated egg when some pooled egg meals and recipes are eliminated, to baseline results for the number of illnesses per contaminated egg.

\section{Results}

At FSI settings, eliminating the use of table eggs in all pooled egg meals and recipes changes the distribution for the number of illnesses per contaminated egg, particularly by reducing the frequency of a large number of illnesses from a single contaminated egg to exactly 0 . Table 25 illustrates the effect by comparing the mean of the distribution of illnesses per contaminated egg under that practice to that of baseline.

Eliminating all pooled egg meals and recipes reduces the number of illnesses per contaminated egg associated with table eggs to $29 \%$ of baseline, but only small gains are made with modest $(\leq 25 \%)$ reductions of pooled egg use. Such large reductions in the number of illnesses only accrue in those egg meals and recipes that do not receive sufficient cooking to inactivate the salmonellae present. Smaller reductions accrue among those egg meals and recipes usually cooked well. Reductions reported in Table 25 accrue from reducing the number of individuals exposed to the $S$. Enteritidis from a contaminated egg, not reducing the prevalence of contaminated eggs.

\section{Sensitivity}

Frequency of pooled egg meals and recipes at FSI settings and the number of servings that pooled egg meals and recipes is not known. Baseline conditions impute expert judgment and anecdotal information.

\section{Discussion}

These results for egg pooling demonstrate the effects of reducing egg prevalence on the number of illnesses per contaminated egg. One means to reduce the frequency of pooled table egg meals and recipes is to substitute processed egg products for shell eggs. The risk associated with $S$. Enteritidis in pasteurized egg products, one means of achieving substitution for table eggs in pooled egg meals, is outside the scope of this risk assessment.

At home settings, pooled egg meals and recipes occur less frequently and involve smaller numbers of servings so that reductions over baseline conditions are smaller than at FSI 
settings (results not shown) and illnesses per contaminated egg are closely represented by the probability of illness from a single serving (section 5.1.2, Table 14).

\subsubsection{Cooking}

\section{Introduction}

Section 5.1.2 Table 15 reports differences in the risk per contaminated egg (probability of illnesses from a serving and the number of illnesses per contaminated egg) among different egg meals and recipes.

\section{Methods}

We evaluate how effective reducing the fraction of raw and lightly cooked egg meals and recipes is on reducing risk by comparing the number of illnesses per contaminated egg under various strategies to baseline conditions.

\section{Results}

Reducing the frequency of egg meals and egg recipes that are consumed raw or lightly cooked reduces the number of illnesses per contaminated egg (Table 26).

At home settings, smaller reductions (96\% to $83 \%$ of baseline) in the number of illnesses per contaminated egg accrue from small reductions to the frequency of raw and lightly cooked egg meals and recipes. Consumption surveys indicate low frequency of raw and lightly cooked egg meals and recipes in home settings (data not shown).

At FSI settings, reductions are larger. Reducing the frequency of raw and lightly-cooked egg meals and recipes by half reduces the number of illnesses per egg to $72 \%$ of baseline.

\begin{tabular}{|c|c|}
\hline $\begin{array}{c}\text { Eliminating pooled egg meals } \\
\text { \& recipes (\%) }\end{array}$ & \% Baseline, at Mean \\
\hline 0 & 100 \\
\hline 25 & 83 \\
\hline 50, & 65 \\
\hline 75 & 48 \\
\hline 100 & 29 \\
\hline
\end{tabular}

Table 25. Effect on the number of illnesses per contaminated table egg from eliminating pooled shell eggs egg meals and recipes in Food Service and Institution settings.

\begin{tabular}{|c|c|c|}
\hline $\begin{array}{c}\text { Eliminating raw and } \\
\text { lightly-cooked meals and } \\
\text { recipes (\%) }\end{array}$ & \multicolumn{2}{|c|}{ \% Baseline, at mean } \\
\cline { 2 - 3 } & FSI settings & Home settings \\
\hline 0 & 100 & 100 \\
\hline 25 & 86 & 96 \\
\hline 50 & 72 & 92 \\
\hline 75 & 58 & 88 \\
\hline 100 & 44 & 83 \\
\hline
\end{tabular}

Table 26. Number of illnesses per contaminated egg as percentage baseline from reducing percentage consumption of lightly cooked and raw egg meals and egg recipes.

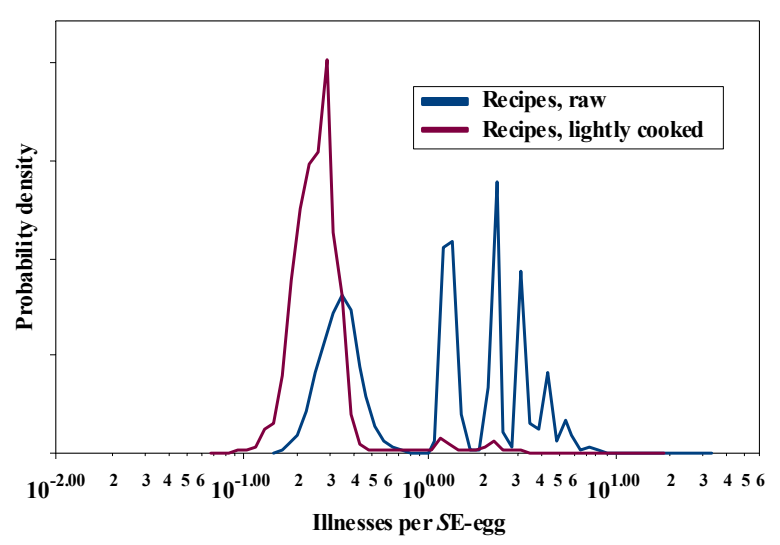

Figure 12. Distribution of the number of illnesses per contaminated egg for raw and lightly cooked recipes.

Eliminating all raw and lightly cooked egg meals and recipes reduces the number of illnesses to $44 \%$ of that under baseline conditions. Particularly, reducing the frequency of raw egg recipes shared among many consumers reduces the frequency of large numbers of illnesses from a single contaminated egg (upper tail, Figure 12). Peaks in the probability density are centred at different cooking effects from the various egg meal and recipe types.

\section{Discussion}

Changing cooking practices within-meal types - shifts away from less well-cooked sunny-side up fried eggs, shifts away from runny scrambled eggs and shifts away from soft-boiled and poached eggs - also reduces the number of illnesses per contaminated egg. Specific strategies were not part of the risk management options assessed.

\subsubsection{Combinations of strategies}

Individual risk mitigations effect particular size reductions in illnesses per contaminated egg when measured in isolation (sections 6.2.1-6.2.3). Table 27 results demonstrate the size of reductions against baseline conditions under combinations of four risk management options (eliminating $50 \%$ of pooled table egg meals; no growth; colder, shorter consumer storage and handling; and reducing the frequency of lightly cooked and raw egg meals and recipes by half).

Each risk mitigation measure makes some incremental reduction to the number of illnesses per contaminated egg, regardless of the baseline against which it is measured, but the amount of incremental reduction depends on what risk mitigations are already accounted for:

- pooled egg replacement $(50 \%)$ has a larger incremental reduction over baseline pooled egg replacement $(0 \%)$ if no steps have been taken to prevent growth $(79 \%$ vs. $100 \%$ baseline; $65 \%$ vs. $81 \%$ baseline; $67 \%$ vs. $82 \%$; $56 \%$ vs. $62 \%$ ), but only a small incremental reduction if mitigation already prevents growth (37\% vs. $38 \%$ baseline; $37 \%$ vs. $38 \%$ baseline; $33 \%$ vs. $34 \%$; $33 \%$ vs. $34 \%)$; 


\begin{tabular}{|c|c|c|c|c|}
\hline $\begin{array}{c}\text { Pooled egg } \\
\text { replacement (\%) }\end{array}$ & $\begin{array}{l}\text { S. Enteritidis growth in } \\
\text { storage \& handling }\end{array}$ & Cooking & Consumer storage & $\%$ of baseline \\
\hline \multirow{8}{*}{ Baseline } & \multirow{4}{*}{ Baseline } & \multirow{2}{*}{ Baseline } & Baseline & 100 \\
\hline & & & Colder, shorter & 81 \\
\hline & & \multirow{2}{*}{$\begin{array}{l}1 / 2 \text { light, } \\
1 / 2 \text { raw }\end{array}$} & Baseline & 82 \\
\hline & & & Colder, shorter & 62 \\
\hline & \multirow{4}{*}{ No growth } & \multirow{2}{*}{ Baseline } & Baseline & 38 \\
\hline & & & Colder, shorter & 38 \\
\hline & & \multirow{2}{*}{$\begin{array}{l}1 / 2 \text { light, } \\
1 / 2 \text { raw }\end{array}$} & Baseline & 34 \\
\hline & & & Colder, shorter & 34 \\
\hline \multirow{8}{*}{50} & \multirow{4}{*}{ Baseline } & \multirow{2}{*}{ Baseline } & Baseline & 79 \\
\hline & & & Colder, shorter & 65 \\
\hline & & \multirow{2}{*}{$\begin{array}{l}1 / 2 \text { light, } \\
1 / 2 \text { raw }\end{array}$} & Baseline & 67 \\
\hline & & & Colder, shorter & 56 \\
\hline & \multirow{4}{*}{ No growth } & \multirow{2}{*}{ Baseline } & Baseline & 37 \\
\hline & & & Colder, shorter & 37 \\
\hline & & \multirow{2}{*}{$\begin{array}{l}1 / 2 \text { light, } \\
1 / 2 \text { raw }\end{array}$} & Baseline & 33 \\
\hline & & & Colder, shorter & 33 \\
\hline
\end{tabular}

Table 27. Effect of combinations of risk mitigations on number of illnesses per $S$. Enteritidis contaminated egg.

- mitigation that prevents $S$. Enteritidis growth has a large incremental reduction regardless of other mitigation measures (38\% vs. $100 \%$ baseline; $38 \%$ vs. $81 \%$ baseline; $34 \%$ vs. $82 \% ; 34 \%$ vs. $62 \% ; 37 \%$ vs. $79 \% ; 37 \%$ vs. $65 \% ; 33 \%$ vs. $65 \% ; 33 \%$ vs. $56 \%$ );

- reducing the proportion of lightly cooked and raw egg dishes by half has a larger incremental reduction when no mitigation measures are in place to prevent growth in $S$. Enteritidis contaminated eggs or to hold eggs at colder temperatures for shorter times at the consumer stage ( $82 \%$ vs. $100 \%$ baseline; $67 \%$ vs. $79 \%$ baseline) than when mitigation measures are in place to prevent growth or to hold eggs at colder temperatures for shorter times (62\% vs. $81 \%$ baseline; $34 \%$ vs. $38 \%$; $34 \%$ vs. $38 \%$; $56 \%$ vs. $65 \%$; $33 \%$ vs. $37 \%$; $33 \%$ vs. $37 \%$ ); and,

- holding eggs at colder storage temperatures for shorter times at the consumer stage has a large reduction when there are no other mitigations to prevent growth of $S$. Enteritidis in contaminated eggs ( $81 \%$ vs. $100 \%$ baseline; $62 \%$ vs. $82 \%$ baseline; $65 \%$ vs. $79 \% ; 56 \%$ vs. $57 \%$ ) than when other mitigation measures already prevent growth $(38 \%$ vs. $38 \%$ baseline; $34 \%$ vs. $34 \%$; $37 \%$ vs. $37 \%$; $33 \%$ vs. $33 \%)$.

That is, incremental effects of several risk management strategies are smaller than the sum of their marginal effects. Further, results indicate the circumstances under which a particular mitigation would have the largest and smallest incremental effect.

\subsubsection{Sensitivity}

\section{Alternative initial $S$. Enteritidis contamination levels}

Initial S. Enteritidis contamination has little influence on risk outputs like the number of illnesses per contaminated egg and the probability of illness from a contaminated egg serving.

\section{Alternative Dose-Response Models}

The dose-response model choice affects risk outputs for illnesses per contaminated egg (Table 18) and the relative influence of risk management options that address prevalence, illnesses per contaminated egg or $S$. Enteritidis levels in an egg serving. Relative to the FAO/WHO [7], USDA [8], or Thomas et al. [11] Beta-Poisson dose-response models, for example, the Weibull dose-response model attributes higher probability of illness at low doses and at high doses for the normal population individuals and at all $S$. Enteritidis doses for the susceptible population individuals (section 6.2).

Relative to that Beta-Poisson model, using the Weibull dose-response model to evaluate risk management options shifts emphasis:

- for the normal population, away from options that address prevalence towards those that address high $S$. Enteritidis levels in an egg serving at consumption;

- for the susceptible population, towards options that address prevalence and away from those that address high $S$. Enteritidis levels in an egg serving at consumption; and,

- among options that address $S$. Enteritidis levels in an egg serving, towards those that mitigate even moderate growth; if the USDA [8] model provides the link between $S$. Enteritidis and human illness, the dose-response function effectively self-limits the probability of illness with increasing dose, so that reducing the use of eggs for pooled egg meals among multiple individuals would lose emphasis as a risk management option relative to risk management options that reduce the frequency of high S. Enteritidis levels. 


\subsection{Discussion points}

Results for baseline conditions (section 5.1.1, 5.1.2) and the examination of sensitivity of the risk outputs to various influential variables (section 5.1.3) pointed to possibilities for risk management: egg prevalence; cooking reduction; pooling practices; storage and handling.

Factors that keep the probability of illness from eating a serving from a contaminated egg small are:

- growth controlled so that $100 \%$ yolk membrane breakdown does not occur and growth from initial deposition at lay does not occur;

- low frequency of occurrence of pooling eggs for egg meals and egg recipes or only small numbers of eggs are used in shared egg meals and egg recipes; and,

- low frequency of lightly cooked or raw egg dishes.

Section 6.2 addresses the effects of preparation practices and storage and handling conditions on the illnesses per contaminated egg.

Factors that keep the probability of illness from eating a serving from an egg at random small are:

- growth controlled so that $100 \%$ yolk membrane breakdown does not occur and growth from initial deposition at lay does not occur;

- low frequency of occurrence of pooling eggs for egg meals and egg recipes or only small numbers of eggs are used in shared egg meals and egg recipes;

- low frequency of lightly cooked or raw egg dishes; and,

- low egg prevalence, through low flock prevalence, low egg within flock prevalence and control of the total number of hens laying in positive flocks.

Section 6.1 addresses the effects of two risk management strategies on the egg prevalence; while section 6.2 examines the effects of preparation practices and storage and handling conditions on the illnesses per contaminated egg.

Under conditions that permit even occasional growth of $S$. Enteritidis, the illnesses per contaminated egg is more influential than the egg prevalence for the number of illnesses. However, as the fraction of contaminated eggs that experience some growth decreases, the influence of egg prevalence relative to the number of illnesses per contaminated egg increases. Under conditions where no growth of $S$. Enteritidis occurs from initial contamination levels, illnesses per contaminated egg and egg prevalence exert the same relative influence on the number of illnesses.

\section{Acknowledgements}

This report was prepared collaboratively between the Bureau of Food Surveillance and Science Integration and the Bureau of Microbial Hazards of the Food Directorate,
Health Products and Food Branch of Health Canada. The members of the Science Team for S. Enteritidis included the following: Normand Boucher (CFIA), Diane Brodeur (EFC), Spencer Collins (EFC), Hélène Couture (HC), Walter Demczuk (PHAC), Leanne DeWinter (HC), Aamir Fazil (PHAC), Thomas Gleeson (HC), Rebecca Irwin (PHAC), Anna Lammerding (PHAC), Steve Matusiak (CFIA), Biljana Mihajlovic (HC), Rachel Ouckama (Industry/CPEPC), Frank Pollari (PHAC), William Ross (HC), Judy Scaife (CFIA), and Victoria Sikur (CHEP). Special thanks to Bernadette Cox and the Egg Farmers of Canada (EFC), who were instrumental in providing access to data regarding regulated commercial layer flocks. Thanks to Angela Catford for useful discussions regarding this risk assessment.

\section{References}

[1] Paoli G (2001), "Risk assessment of Salmonella Enteritidis in Canadian shell eggs", Health Canada, unpublished, Decisionalysis.

[2] Health Canada (2000), "Health Canada's Decision Making Framework". Available at: http://www.hcsc.gc.ca/ahc-asc/pubs/hpfb-dgpsa/risk-risques_cppc_e.html (Accessed Feb. 25, 2008).

[3] Codex Alimentarius Commission (1999), "Principles and guidelines for the conduct of microbiological risk assessment". Available at:

www.codexalimentarius.net/download/standards/357 /CXG_030e.pdf (Accessed Feb. 25, 2008).

[4] Whiting RC \& RL Buchanan (1997), “Development of a quantitative risk assessment model for Salmonella Enteritidis in pasteurized liquid eggs", Intl J. Food Microbiol. 36, 111-125.

[5] Whiting RC, A Hogue, WD Schlosser, ED Ebel, RA Morales, A Baker \& RM McDowell (2000), “A quantitative process model for Salmonella Enteritidis in shell eggs", J Food Sci. 65 (5), 864-869.

[6] Hope BK, AR Baker, ED Ebel, AT Hogue, WD Schlosser, R Whiting, RM McDowell \& RA Morales (2002), "An overview of the Salmonella Enteritidis risk assessment for shell eggs and egg products", Risk Anal. 22 (2), 203-218.

[7] Food and Agriculture Organization of the United Nations and World Health Organization (FAO/WHO) (2002), "Risk assessments of Salmonella in eggs and broiler chickens", ISBN 9291562293 (WHO), ISBN 92 5104872 X (FAO) ISSN 1726-5274.

[8] US Department of Agriculture, Food Safety \& Inspection Service (USDA) (2005), "Risk assessments of Salmonella Enteritidis in shell eggs and Salmonella spp. in egg products".

[9] Lievonen S, J Ranta \& R Maijala (2006), "Salmonella in egg production in Finland -a quantitative risk assessment", publication 04/2006, National Veterinary and Food Research Institute, Helsinki, Finland. 
[10] Mokhtari A, CM Moore, H Yang, L-A Jaykus, R Morales, SC Cates \& P Cowen (2006), “Consumerphase Salmonella enterica serovar Enteritidis risk assessment for egg-containing food products", Risk Anal. 26 (3), 753-768.

[11] Thomas C, B Daughtry, D Padula, D Jordan, G Arzey, K Davey, G Holds, J Slade \& A Pointon (2006c), “An egg:Salmonella quantitative risk assessment model for the Australian egg industry", AECL Publication No 06/AECL Project SAR-42A, ISSN 1448-1316.

[12] Kelly L, L Murchie, B Xia, P Whyte \& RH Madden (2009), "Probabilistic model for contamination of egg dishes with Salmonella spp. Made from shell eggs produced on the island of Ireland", Intl J. Food Microbiol. 135, 187-192.

[13] European Food Safety Authority (2010a), "Quantitative risk assessment of Salmonella Enteritidis in shell eggs in Europe", EFSA J. 8 (4), 1588, EFSA-Q2009-00790,

http://www.efsa.europa.eu/en/scdocs/scdoc/1588.htm [accessed 2010.May.01].

[14] European Food Safety Authority (2010b), “Scientific opinion on the quantitative estimation of the public impact of setting a new target for the reduction of Salmonella in laying hens", EFSA J. 8 (4), 1546, EFSAQ-2008-292,

http://www.efsa.europa.eu/en/scdocs/scdoc/1546.htm [accessed 2010.May.01].

[15] Mead PS, Slutsker L, Dietz V, McCaig LF, Bresee JS, Shapiro C, Griffin PM \& RV Tauxe (1999), "Foodrelated illness and death in the United States", Emerg Infect Dis. 5 (5), 607-25.

[16] Department of Justice Canada (2009), “Egg Regulations C.R.C., c.284", Part 1, Section 9 (31) to (33). Available at:

http://laws.justice.gc.ca/en/showdoc/cr/C.R.C.c.284///en?page=1 (Accessed Feb. 25, 2008).

[17] Centers for Disease Control and Prevention (CDC) (2010), "Investigation update: multistate outbreak of human Salmonella Enteritidis infections associated with shell eggs". Available at:

http://www.cdc.gov/print.do?url=http\%3A//www.cdc .gov/salmonella/enteritidis/ (Accessed Dec. 7, 2010).

[18] Centers for Disease Control and Prevention (CDC) (2003), "Salmonella serotype Enteritidis outbreak summaries". Available at:

http://www.cdc.gov/foodborneoutbreaks/outbreak_da ta.htm (Accessed Feb. 25, 2008).

[19] Patrick ME, Adcock PM, Gomez TM, Altekruse SF, Holland BH, Tauxe RV \& DL Swerdlow (2004), "Salmonella Enteritidis infections, United States, 19851999", Emerg Infect Dis. 10 (1), 1-7.

[20] Voetsch AC, Poole C, Hedberg CW, Hoekstra RM, Ryder RW, Weber DJ \& FJ Angulo (2008), “Analysis of the FoodNet case-control study of sporadic Salmonella serotype Enteritidis infections using persons infected with other Salmonella serotypes as the comparison group", Epidemiol Infect. 137, 408-416.

[21] Public Health Agency of Canada (PHAC) (2004), "Laboratory Surveillance Data for Enteric Pathogens in Canada, Annual Summary 2001". Available at: http://www.nml-lnm.gc.ca/english/NESP.htm

(Accessed March 3, 2008)

[22] Public Health Agency of Canada (PHAC) (2008), "National Notifiable Diseases On-Line". Available at: http://dsol-smed.phac-aspc.gc.ca/dsolsmed/ndis/list_e.html (Accessed March 3, 2008).

[23] Medaglia A (2008), personal communication, e-mail Feb. 26, 2008; Database Manager, Notifiable Diseases Section, Surveillance and Risk Assessment Division, Public Health Agency of Canada.

[24] Khakhria R, Woodward D, Johnson WM \& C Poppe (1997), "Salmonella isolated from humans, animals and other sources in Canada, 1983-92", Epidemiol Infect. 119 (1), 15-23.

[25] Health Canada (2003), “Canadian Integrated Surveillance Report: Salmonella, Campylobacter, pathogenic E. coli, and Shigella, from 1996 to 1999". Can. Commun. Dis. Rep. 2951.

[26] Public Health Agency of Canada (PHAC) (2005), "Laboratory Surveillance Data for Enteric Pathogens in Canada, Annual Summary 2002 and 2003". Available at: $\quad$ http://www.nml-lnm.gc.ca/english/NESP.htm (Accessed March 3, 2008).

[27] Public Health Agency of Canada (PHAC) (2007a), Laboratory Surveillance Data for Enteric Pathogens in Canada, Annual Summary 2005. Available at: http://www.nml-lnm.gc.ca/english/NESP.htm (Accessed March 3, 2008).

[28] Public Health Agency of Canada (PHAC) (2007b), Laboratory Surveillance Data for Enteric Pathogens in Canada, Annual Summary 2006. Available at: http://www.nml-lnm.gc.ca/english/NESP.htm (Accessed March 3, 2008).

[29] Thomas MK, SE Majowicz, L MacDougall, PN Sockett, SJ Kovacs, M Fyfe, VL Edge, K Doré, JA Flint, $S$ Henson \& AQ Jones (2006a), "Population distribution and burden of acute gastrointestinal illness in British Columbia, Canada", BMC Pub Health 6, 307.

[30] Thomas MK, Majowicz SE, Socket PN, Fazil A, Pollari F, Doré K, Flint JA \& VL Edge (2006b), "Estimated numbers of community cases of illness due to Salmonella, Campylobacter and verotoxigenic Escherichia coli: pathogen-specific community rates", Can J Infect Dis Med Microbiol. 17 (4), 229-234.

[31] Public Health Agency of Canada (PHAC) (2002a), "Results of a Physician Study Pilot in the New City of Hamilton Region". Available at: http://www.phacaspc.gc.ca/nsagi-enmga/pdf/phys_pilot_e.pdf (Accessed Aug. 13, 2008). 
[32] Public Health Agency of Canada (PHAC) (2002b), "Report of the 2001 Canadian Laboratory Survey, National Studies on Acute Gastrointestinal Illness", http://www.phac-aspc.gc.ca/nsagi-

enmga/pdf/labstudyreport_e.pdf (Accessed Aug. 13, 2008).

[33] Flint JA, K Doré, SE Majowicz, VL Edge \& P Sockett (2004), "From stool to statistics. Reporting of acute gastrointestinal illnesses in Canada", Can. J Publ Health 95 (4), 309-313.

[34] Majowicz SE, K Doré, JA Flint, VL Edge, S Read, MC Buffett, S McEwen, WB McNab, D Stacey, P Sockett \& JB Wilson (2004), "Magnitude and distribution of acute, self-reported gastrointestinal illness in a Canada community", Epidemiol Infect. 132, 607-617.

[35] Majowicz SE, VL Edge, A Fazil, WB McNab, KA Doré, PN Sockett, JA Flint, D Middleton, SA McEwen \& JB Wilson (2005), "Estimating the under-reporting rate for infectious gastrointestinal ilness in Ontario", Can. J. Publ. Health 96 (3), 178-181.

[36] Boucher, N. Chief, Hatchery Program, CFIA. Personal communication. April 18, 2008.

[37] Canadian Egg Marketing Agency (2002), "Beyond 30 Years - 2002 Annual Report". Available at: http://publications.gc.ca/site/eng/344664/publication.h tml (Accessed April 13, 2011).

[38] Egg Farmers of Canada. 19 March 2009. Personal communication [Conference call].

[39] Canadian Egg Marketing Agency (CEMA) (2007a), personal communication, e-mails 2007.February.19, 2007.March.20, 2008.April.04, 2008.April.23.

[40] Davies R \& M Breslin (2003b), “Effects of vaccination and other preventive methods for Salmonella Enteritidis on commercial laying chicken farms", Vet Rec. 153 (22), 673-677.

[41] Cogan TA \& TJ Humphrey (2003), "The rise and fall of Salmonella Enteritidis in the UK", J Appl Microbiol. 94 Suppl, 114S-119S.

[42] Gast RK (2007), "Serotype-specific and serotypeindependent strategies for preharvest control of foodborne Salmonella in poultry", Avian Dis. 51, 817-28.

[43] Gast RK \& PS Holt (2001), “Assessing the frequency and consequences of Salmonella enteritidis deposition on the egg yolk membrane", Poult Sci. 80 (7), 997-1002.

[44] Gast RK, Guraya R, Guard-Bouldin J, Holt PS \& RW Moore (2007), "Colonization of specific regions of the reproductive tract and deposition at different locations inside eggs laid by hens infected with Salmonella enteritidis or Salmonella heidelberg", Avian Dis. 51 (1), 40-4.

[45] Gantois I, Ducatelle R, Pasmans F, Haesebrouck F, Gast R, Humphrey TJ \& F Van Immerseel (2009), "Mechanisms of egg contamination by Salmonella Enteritidis", FEMS Microbiol Rev. 33 (4), 718-38.
[46] Fleischman GJ, Napier CL, Stewart D \& SA Palumbo (2003), "Effect of temperature on the growth response of Salmonella enteritidis inoculated onto the vitelline membranes of fresh eggs", J Food Prot. 66 (8), 1368-73.

[47] Chen J, Shallo Thesmar H \& WL Kerr (2005), "Outgrowth of salmonellae and the physical property of albumen and vitelline membrane as influenced by egg storage conditions", J Food Prot. 68 (12), 2553-8.

[48] Poppe C, Irwin RJ, Forsberg CM, Clarke RC \& J Oggel (1991a), "The prevalence of Salmonella enteritidis and other Salmonella spp. among Canadian registered commercial layer flocks", Epidemiol Infect. 106, 259-70.

[49] Poppe C, RP Johnson, CM Forsberg \& RJ Irwin (1992), "Salmonella enteritidis and other Salmonella in laying hens and eggs from flocks with Salmonella in their environment", Can. J. Vet. Res. 56 (3), 226-232.

[50] Saskatchewan Egg Producers (SEP) (2003), “Annual Report". Available at: http://www.agr.gov.sk.ca/agrifood/boards/SKEggPro ducers.htm (Accessed Aug. 15, 2008).

[51] Ontario Egg Producers (OEP) (2004), “2004 Annual Report". Available at: http://www.getcracking.ca/PDF/2004_Annual_Report _FINAL.pdf (Accessed Dec. 9, 2010)

[52] Ontario Egg Producers (OEP) (2005), “2005 Annual Report". Available at: http://www.getcracking.ca/PDF/OEPAnnualReport20 05FINAL.pdf (Accessed Aug. 15, 2008)

[53] Fédération des Producteurs d'Oeufs de Consommation du Québec. 2006. Rapport annuel 2005-2006 de la Fédération des producteurs d'oeufs de consommation du Québec. À l'heure des défis. http://www.oeufs.ca/en/publications/documents/detai ls/index.asp?RubriqueID=10\&DocID=37 (Accessed Sept. 11, 2006).

[54] Canadian Egg Marketing Agency (CEMA) (2007b), personal communication, e-mail 2007.December.20

[55] Poppe C, Irwin RJ, Messier S, Finley GG \& J Oggel (1991b), "The prevalence of Salmonella enteritidis and other Salmonella sp. among Canadian registered commercial chicken broiler flocks", Epidemiol Infect. 107, 201-11.

[56] Guerin MT, Martin SW, Darlington GA \& A Rajic (2005), "A temporal study of Salmonella serovars in animals in Alberta between 1990 and 2001", Can J Vet Res. 69 (2), 88-99.

[57] Ouckama R (2006), personal communication, e-mail Oct. 13, 2006.

[58] Davies R \& M Breslin (2001), “Environmental contamination and detection of Salmonella enterica serovar enteritidis in laying flocks", Vet Rec. 149 (23), 699-704.

[59] Henzler DJ, Ebel E, Sanders J, Kradel D \& J Mason (1994), "Salmonella enteritidis in eggs from commercial chicken layer flocks implicated in human outbreaks", Avian Dis. 38 (1), 37-43. 
[60] Kinde H, Castellan DM, Kerr D, Campbell J, Breitmeyer R \& A Ardans (2005), “Longitudinal monitoring of two commercial layer flocks and their environments for Salmonella enterica serovar enteritidis and other Salmonellae", Avian Dis. 49 (2), 189-94.

[61] Henzler DJ, Kradel DC \& WM Sischo (1998), "Management and environmental risk factors for Salmonella enteritidis contamination of eggs", Am J Vet Res. 59 (7), 824-9.

[62] Lister SA (1988), "Salmonella enteritidis infection in broilers and broiler breeders", Vet Rec. 123 (13), 350.

[63] Bygrave AC \& J Gallagher (1989), "Transmission of Salmonella enteritidis in poultry", Vet Rec. 124 (21), 571.

[64] Saskatchewan Egg Producers (SEP) (2004), “Annual Report". Available at:

http://www.agr.gov.sk.ca/agrifood/boards/SKEggPro ducers.htm (Accessed Aug. 15, 2008).

[65] Humphrey TJ, Baskerville A, Mawer S, Rowe B \& S Hopper (1989), "Salmonella enteritidis phage type 4 from the contents of intact eggs: a study involving naturally infected hens", Epidemiol Infect. 103 (3), 415-23.

[66] Humphrey TJ (1994), "Contamination of egg shell and contents with Salmonella Enteritidis: a review", Intl J. Food Microbiol. 21 (1-2), 31-40.

[67] Mason (1994), "Salmonella Enteritidis control programs in the United States", Intl J. Food Micorbiol. 21 (1-2), 155-169.

[68] Ebel E \& W Schlosser (2000), "Estimating the annual fraction of eggs contaminated with Salmonella Enteritidis in the United States", Intl J. Food Microbiol. 61 (1), 51-62.

[69] Davies R \& M Breslin (2004), “Observations on Salmonella contamination of eggs from infected commercial laying flocks where vaccination for Salmonella enterica serovar Enteritidis had been used" Avian Pathol. 33 (2), 133-144.

[70] Piao Z, Toyota-Hanatani Y, Ohta H, Sasai K, Tani H \& E Baba (2007), "Effects of Salmonella enterica subsp. enterica serovar Enteritidis vaccination in layer hens subjected to $S$. Enteritidis challenge and various feed withdrawal regimens", Vet Microbiol. 125 (1-2), 111-9.

[71] Humphrey TJ, Whitehead A, Gawler AH, Henley A \& B Rowe (1991), "Numbers of Salmonella enteritidis in the contents of naturally contaminated hens' eggs", Epidemiol Infect. 106 (3), 489-96.

[72] Gast RK \& CW Beard (1992), "Evaluation of a chick mortality model for predicting the consequences of Salmonella enteritidis infections in laying hens", Poult Sci. 71 (2), 281-7.

[73] Humphrey TJ \& A Whitehead (1993), “Egg age and the growth of Salmonella enteritidis PT4 in egg contents", Epidemiol Infect. 111 (2), 209-19.

[74] Cogan TA, Domingue G, Lappin-Scott HM, Benson CE, Woodward MJ \& TJ Humphrey (2001), “Growth of Salmonella enteritidis in artificially contaminated eggs: the effects of inoculum size and suspending media", Int J Food Microbiol. 70 (1-2), 131-41.
[75] Gast RK, Holt PS \& R Guraya (2006), “Effect of refrigeration on in vitro penetration of Salmonella enteritidis through the egg yolk membrane", J Food Prot. 69 (6), 1426-9.

[76] Bradshaw JG, DB Shah, E Forney \& JM Madden (1990), "Growth of Salmonella Enteritidis in yolk of shell eggs from normal and seropositive hens", J Food Protect. 53 (12), 1033-1036.

[77] Schoeni JL, Glass KA, McDermott JL \& AC Wong (1995), "Growth and penetration of Salmonella enteritidis, Salmonella heidelberg and Salmonella typhimurium in eggs", Int J Food Microbiol. 24 (3), 385396.

[78] Latimer HK, L-A Jaykus, RA Morales, P Cowen \& D Crawford-Brown (2002), "Sensitivity analysis of Salmonella Enteritidis levels in contaminated shell eggs using a biphasic growth model", Int J Food Microbiol. 75, 71-87.

[79] Sockett PN, Cowden JM, Le Baigue S, Ross D, Adak GK \& H Evans (1993), “Foodborne disease surveillance in England and Wales: 1989-1991", Commun Dis Rep CDR Rev. 3 (12), R159-73.

[80] Palumbo MS, SM Beers, S Bhaduri \& SA Palumbo (1995), "Thermal resistance of Salmonella spp. and Listeria monocytogenes in liquid egg yolk and egg yolk products", J Food Prot. 58 (9), 960-6.

[81] Blackburn C, LM Curtis, L Humpheson, C Billon \& PJ McClure (1997), “Development of thermal inactivation models for Salmonella Enteritidis and Escherichia coli O157: $\mathrm{H7}$ with temperature, $\mathrm{pH}$ and $\mathrm{NaCl}$ as controlling factors", Int J Food Microbiol. 38, 31-44.

[82] Chantarapanont W, Slutsker L, Tauxe RV \& LR Beuchat (2000), "Factors influencing inactivation of Salmonella Enteritidis in hard-cooked eggs", J Food Protect. 63 (1), 36-43.

[83] Grijspeerdt K \& L Herman (2003), "Inactivation of Salmonella enteritidis during boiling of eggs", Int J Food Microbiol. 82 (1), 13-24.

[84] Mañas P, Pagán R, Raso J \& S Condón (2003), "Predicting thermal inactivation in media of different $\mathrm{pH}$ of Salmonella grown at different temperatures", Int J Food Microbiol. 87 (1-2), 45-53.

[85] De Paula CMD, RF Mariot \& EC Tondo (2005), "Thermal inactivation of Salmonella Enteritidis by boiling and frying egg methods", J Food Safety 25, 43-57.

[86] Agriculture and Agri-Foods Canada (AAFC) (2006), "Canada's Egg Industry",

http://www.agr.gc.ca/poultry, [accessed 2008.February.25].

[87] Kennedy M, Villar R, Vugia DJ, Rabatsky-Ehr T, Farley MM, Pass M, Smith K, Smith P, Cieslak PR, Imhoff B, Griffin PM, \& Emerging Infections Program FoodNet Working Group (2004), “Hospitalizations and deaths due to Salmonella infections, FoodNet, 1996-1999", Clin Infect Dis. 38 (Suppl 3), S142-8.

[88] Vugia DJ, Samuel M, Farley MM, Marcus R, Shiferaw B, Shallow S, Smith K, Angulo FJ \& 
Emerging Infections Program FoodNet Working Group (2004), "Invasive Salmonella infections in the United States, FoodNet, 1996-1999: incidence, serotype distribution, and outcome", Clin Infect Dis. 38 (Suppl 3), S149-56.

[89] Weinberger M, Andorn N, Agmon V, Cohen D, Shohat T \& SD Pitlik (2004), "Blood invasiveness of Salmonella enterica as a function of age and serotype", Epidemiol Infect. 132 (6), 1023-8.

[90] Arshad MM, Wilkins MJ, Downes FP, Rahbar MH, Erskine RJ, Boulton ML, Younus M, \& AM Saeed (2008), "Epidemiologic attributes of invasive nontyphoidal Salmonella infections in Michigan, 19952001", Int J Infect Dis. 12 (2), 176-82.

[91] Jones TF, Ingram LA, Cieslak PR, Vugia DJ, TobinD'Angelo M, Hurd S, Medus C, Cronquist A \& FJ Angulo (2008), "Salmonellosis outcomes differ substantially by serotype", J Infect Dis. 198 (1), 109-14.

[92] Rajda Z \& D Middleton (2006), "Descriptive epidemiology of enteric illness for selected reportable diseases in Ontario, 2003", Can Commun Dis Rep. 32 (23), 275-85.

[93] Ross et al. Personal communication. 2000.

[94] Hennessy TW, Hedberg CW, Slutsker L, White KE, Besser-Wiek JM, Moen ME, Feldman J, Coleman WW, Edmonson LM, MacDonald KL, Osterholm MT (1996), "A national outbreak of Salmonella enteritidis infections from ice cream. The Investigation Team", N Engl J Med. 334(20), 1281-6.

[95] Vought KJ \& SR Tatini, (1998), “Salmonella enteritidis contamination of ice cream associated with a 1994 multistate outbreak", J. Food Protect. 61, 5-10.

[96] Anonymous (1971), “A collaborative report: A waterborne epidemic of salmonellosis in Riverside, California, 1965. Epidemiologic aspects", Amer. J. Epidemiol. 93 (1), 33-48.

[97] Federal-Provincial Nutrition Surveys (1991-1999), Health Canada, Food Directorate, Bureau of Nutritional Sciences and Bureau of Food Policy and Science Integration.
[98] Audits International (1999), “U.S. food temperature evaluation". Available at: http://www.foodrisk.umd.edu/exclusives/audits/downloa ds/Audits-FDA_temp_study.pdf (Accessed Feb. 25, 2008).

[99] O'Brien (1997), "Domestic refrigerator air temperatures and the public's awareness of refrigerator use", Int J Environ Health Res. 7, 141-148.

[100] Sergelidis D, Abrahim A, Sarimvei A, Panoulis C, Karaioannoglou P \& C Genigeorgis (1997), “Temperature distribution and prevalence of Listeria spp. in domestic, retail and industrial refrigerators in Greece", Int J Food Microbiol. 34 (2), 171-7.

[101] Johnson, Donkin, Morgan, Lilley, Neale, Page \& Silburn (1998), "Food safety knowledge and practice among elderly people living at home", J Epidemiol Community Health 52, 745-748.

[102] Lievonen S, Havulinna AS \& R Maiala (2004), “Egg consumption patterns and Salmonella risk in Finland", J Food Prot. 67 (11), 2416-23.

[103] Statistics Canada (2004), Canadian Community Health Survey 2.2, 24-hour dietary recall component, http://www.statcan.gc.ca/cgibin/imdb/p2SV.pl?Function=getSurvey\&SDDS=5049\& lang $=\mathrm{en} \& \mathrm{db}=\mathrm{imdb} \& \mathrm{adm}=8 \& \mathrm{dis}=2 \quad$ [accessed 2010.January.04].

[104] Namata H, E Méroc, M Aerts, C Faes, JC Abrahantes, H Imberechts \& K Mintiens (2008), "Salmonella in Belgian laying hens: an identification of risk factors", Prev. Vet. Med. 83 (3-4), 323-336.

[105] Lee R, ME Beatty, AK Bogard, M-P Esko, FJ Angulo, C Selman \& EHS-NET Working Group (2004), "Prevalence of high-risk egg-preparation practices in restaurants that prepare breakfast egg entreés: an EHS-Net study", J. Food Protect. 67 (7), 1444-1450. 